\title{
Strategies for improvement of population based breast cancer screening
}

Citation for published version (APA):

Klompenhouwer, E. G. (2015). Strategies for improvement of population based breast cancer screening. [Doctoral Thesis, Maastricht University]. Maastricht University. https://doi.org/10.26481/dis.20151023ek

Document status and date:

Published: 01/01/2015

DOI:

10.26481/dis.20151023ek

Document Version:

Publisher's PDF, also known as Version of record

\section{Please check the document version of this publication:}

- A submitted manuscript is the version of the article upon submission and before peer-review. There can be important differences between the submitted version and the official published version of record.

People interested in the research are advised to contact the author for the final version of the publication, or visit the DOI to the publisher's website.

- The final author version and the galley proof are versions of the publication after peer review.

- The final published version features the final layout of the paper including the volume, issue and page numbers.

Link to publication

\footnotetext{
General rights rights.

- You may freely distribute the URL identifying the publication in the public portal. please follow below link for the End User Agreement:

www.umlib.nl/taverne-license

Take down policy

If you believe that this document breaches copyright please contact us at:

repository@maastrichtuniversity.nl

providing details and we will investigate your claim.
}

Copyright and moral rights for the publications made accessible in the public portal are retained by the authors and/or other copyright owners and it is a condition of accessing publications that users recognise and abide by the legal requirements associated with these

- Users may download and print one copy of any publication from the public portal for the purpose of private study or research.

- You may not further distribute the material or use it for any profit-making activity or commercial gain

If the publication is distributed under the terms of Article $25 \mathrm{fa}$ of the Dutch Copyright Act, indicated by the "Taverne" license above, 
Strategies for improvement of population based breast cancer screening 
Cover design: promotie in zicht (www.promotie-inzicht.nl)

Lay-out: H.A.M.E. Wouters-Lenssen

Printed by: Ipskamp drukkers B.V.

ISBN: 978-94-6259-787-7

(C) E.G. Klompenhouwer, 2015

All rights reserved. No part of this publication may be reproduced, stored in a retrieval system or transmitted in any form or by any means without permission of the author, or, when appropriate, of the publishers of the publications.

The publication of this thesis was financially supported by:

Catharina ziekenhuis Eindhoven; Bevolkingsonderzoek Zuid; Bracco imaging Europe B.V.; Maatschap radiologie, Catharina ziekenhuis Eindhoven; Guerbet Nederland B.V; Tromp Medical B.V.; Biotronik Nederland B.V.

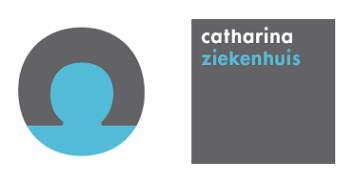

bevolkingsonderzoek
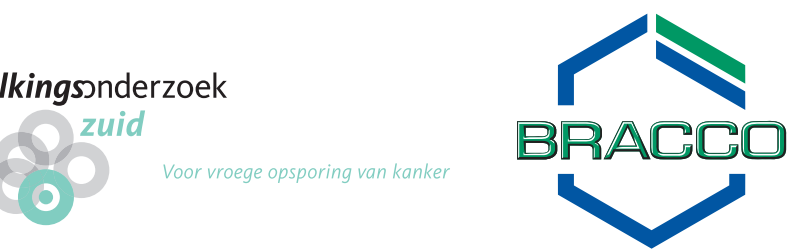

LIFE FROM INSIDE

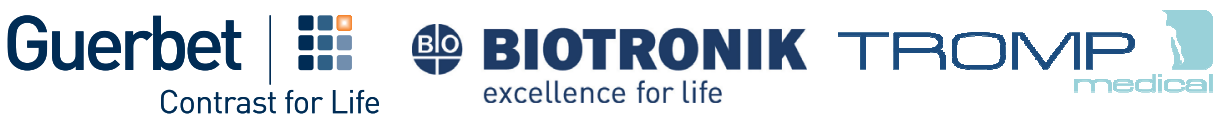




\title{
Strategies for improvement of population based
}

\section{breast cancer screening}

\author{
PROEFSCHRIFT \\ ter verkrijging van de graad van doctor \\ aan de Universiteit Maastricht, \\ op gezag van de Rector Magnificus, Prof. dr. L.L.G. Soete, \\ volgens het besluit van het College van Decanen, \\ in het openbaar te verdedigen op \\ op vrijdag 23 oktober 201516.00
}

door

Elisabeth Geneviève Klompenhouwer

Geboren op 22 februari 1985 te Rotterdam 


\section{Promotor}

Prof. dr. V.C.G. Tjan-Heijnen

\section{Copromotoren}

Dr. L.E.M. Duijm (Canisius Wilhelmina Ziekenhuis)

Dr. M.J.M Broeders (Radboud Universitair Medisch Centrum)

Dr. A.C. Voogd

\section{Beoordelingscommissie}

Prof. dr. C.H.C. Dejong, voorzitter

Dr. M.B.I. Lobbes

Dr. R.M. Pijnappel (Universitair Medisch Centrum Utrecht)

Prof. dr. A.L.M. Verbeek (Radboud Universitair Medisch Centrum) 


\section{Contents}

Chapter 1 General introduction

Chapter 2 Re-attendance at biennial screening mammography following a repeated false positive recall

Chapter 3 Variations in screening outcome among pairs of screening radiologists at non-blinded double reading of screening mammograms: a population-based study

4.1 Blinded double reading yields a higher program sensitivity than non-blinded double reading at digital screening mammography: a prospective population-based study in the south of the Netherlands

4.2 Comparison of the diagnostic workup of women recalled at non-blinded or blinded double reading in a population-based screening mammography program in the South of The Netherlands

4.3 Discrepant screening mammography assessments at blinded and non-blinded double reading: impact of arbitration by a third reader on screening outcome

4.4 Arbitration of discrepant BI-RADS 0 recalls by a third reader at screening mammography lowers recall rate but not the cancer detection rate and sensitivity at blinded and non-blinded double reading

Chapter 5 Discussion and future perspectives: strategies for improvement of population-based breast cancer screening

Chapter $6 \quad 6.1$ Summary

6.2 Valorization

6.3 Curriculum vitae

6.4 List of publications 



\section{Chapter 1}

General introduction 
There is an ongoing debate on the effectiveness and efficacy of breast cancer screening. In January 2014, a committee of the Health Council of the Netherlands concluded that, in the Netherlands, population based screening for breast cancer continues to be as effective as was originally expected, even though the therapeutic landscape has changed since its introduction 25 years ago. ${ }^{1}$ To continue to keep this balance in favor of screening one should try to improve the efficacy of the breast screening program in the Netherlands and minimize the harms.

This thesis will explore several aspects of the breast cancer screening program in a Southern screening region of the Netherlands with the aim to improve the effectiveness of screening. The study population consists of a consecutive series of women screened since 1997 in the South of the Netherlands. The main focus of this thesis will be the evaluation of patients with a repeated false positive recall at the transition to digital screening and its effect on screening re-attendance, radiologist's performance at non-blinded double reading and to determine the best reading strategy at double reading.

\section{Breast cancer incidence}

Breast cancer is the most frequently diagnosed cancer in women worldwide. ${ }^{2}$ Also in the Netherlands, breast cancer is an important threat for public health as the incidence is among the highest in Europe, and still rising (Figure 1.1). ${ }^{3}$ Several causes contribute to the increased incidence, including the introduction of the national breast cancer screening program (with the detection of many in situ carcinoma's), increased awareness of breast cancer among women, and unfavorable developments in the exposure to risk factors for breast cancer. ${ }^{1,4}$

Figure 1.1 Breast cancer incidence in the Netherlands

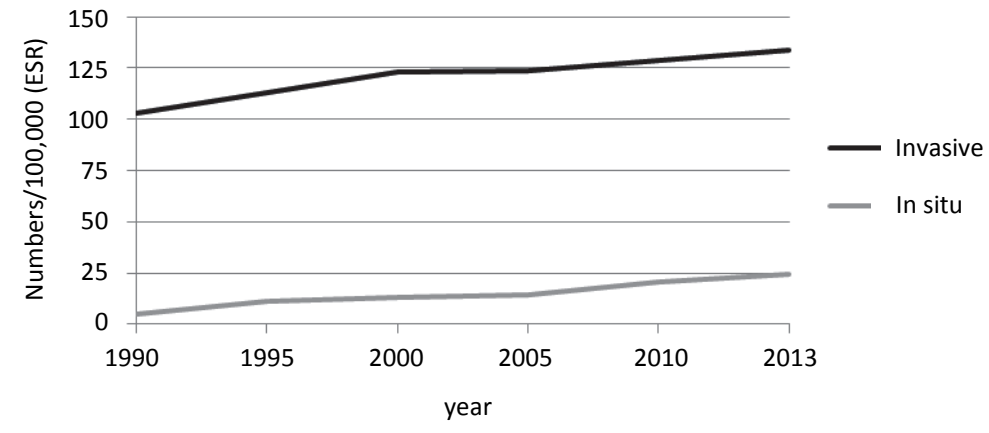

Source: www.cijfersoverkanker.nl 


\section{Breast cancer screening in the Netherlands}

As in many other countries, the Netherlands has introduced a population based screening program with the aim to reduce breast cancer mortality by detecting the disease at an early stage. Since the introduction of breast cancer screening in the Netherlands in 1989 the breast cancer mortality has decreased substantially (Figure 1.2). However, population screening for breast cancer was introduced at a time of fundamental improvements in the treatment of breast cancer and women have become more aware of breast cancer. Therefore, the exact contribution of each improvement on breast cancer mortality is difficult to determine. ${ }^{1,5,6}$

Figure 1.2 Breast cancer mortality in the Netherlands

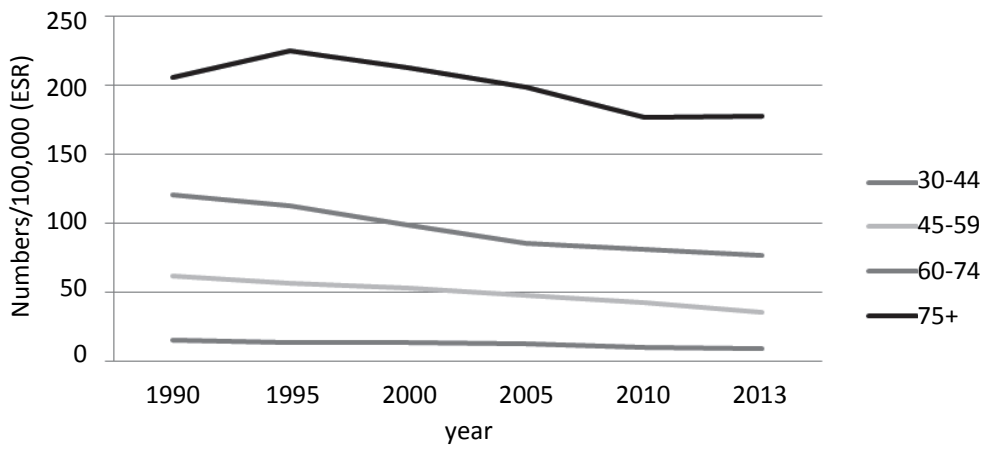

Source: www.cijfersoverkanker.nI

Breast cancer screening in the Netherlands was introduced in 1989 for women aged 50-69 years. Since 1998, the Dutch breast cancer screening program offers biennial mammography to women aged $50-75$ years. A two-view mammogram is obtained by a certified screening technologist and independently read by two qualified screening radiologists. In the Netherlands the initial results of the screening examination are sent to the general practitioner, who serves as an intermediary between the screening organization and healthcare system. In case of an abnormal screening examination, the general practitioner refers the patient to a hospital where she will be analyzed in a multidisciplinary breast cancer team (Figure 1.3).

The Breast Imaging Reporting and Data System (BI-RADS) ${ }^{7}$ has been adapted for use in the screening program in 2009-2010 and provides insight into the likelihood of breast cancer being present. A pilot study in the Amsterdam screening region ${ }^{8}$ in the period 2005-2006 showed that BI-RADS was a suitable tool for stratifying women according to the radiologist's suspicion for malignancy. In the Netherlands, women with a BI-RADS score of 0,4 or 5 are recalled and further evaluated at a dedicated hospital breast unit (Figure 1.3). BI-RADS category 0 represents a possible finding needing additional work-up, BI-RADS category 4 represents a suspicious abnormality with a 
reasonable possibility of being malignant but not the classic appearance of malignancy and BI-RADS 5 category represents an abnormality highly suggestive for malignancy. ${ }^{8}$

Figure 1.3 Organization of Breast cancer screening in the Netherlands

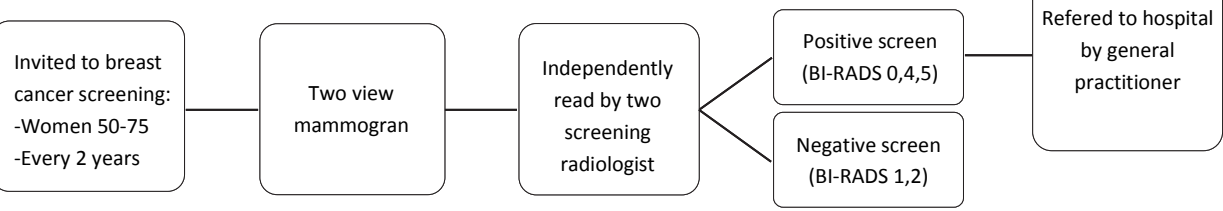

\section{Screening outcome}

Screening can only be effective if certain criteria are met, such as a sufficiently high attendance, referral- and detection rate, a sufficiently high sensitivity and specificity and a relatively low rate of interval cancers and false positive screening results. Screening programs are evaluated based on these parameters. The key performance indicators of the breast cancer screening program in the Netherlands in 1998 and 2012 are shown in Table 1.1. After the introduction of breast cancer screening several important alterations took place with the aim to improve the efficiency of the nationwide screening program, as is reflected by a change in key performance indicators. These alterations are discussed below.

Table 1.1 Key performance indicators of the breast cancer screening program in the Netherlands in 1998 and $2012^{9}$

\begin{tabular}{lcc} 
& 1998 & 2012 \\
\hline Number of women invited & 832,470 & 1266,555 \\
Participation rate (\%) & 80.1 & 79.7 \\
Re-attendance rate (\%) & 91.8 & 92.1 \\
Recall rate per 1000 screened women & 9.9 & 23.5 \\
Cancer detection per 1000 screened women & 4.8 & 6.6 \\
DCIS (\%) & 14.6 & 20.5 \\
False positive per 1000 screened women & & 11.1 \\
After non-invasive diagnostics & 2.4 & 5.6 \\
After invasive diagnostics & 2.5 & 28.0 \\
Positive predictive value of recall (\%) & 48.4 & $2.2^{*}$ \\
Interval cancers per 1000 screened women & 2.1 & $73.0^{*}$ \\
Program sensitivity (\%) & 68.2 & $98.7^{*}$ \\
Program specificity (\%) & 99.3 &
\end{tabular}

DCIS:ductal carcinoma in situ. * The interval cancer numbers from 2012 are not yet available, therefore the interval cancer rate, program sensitivity/specificity are obtained from 2009. 


\section{Women invited and (re-)attendance}

The target population, and thereby the number of women invited, increased with $50 \%$ between 1998 and 2012. This is mainly due to a general rise in population and survival and boosted by the post-war baby-boomers. Currently, 1.2 million women are invited every year to participate in the breast cancer screening program, with a relative stable attendance rate of $80 \%$ and re-attendance rate of more than $90 \% .{ }^{10}$

\section{Recall rate}

The number of women recalled for further assessment as a proportion of all women who had a screening examination is expressed as the recall rate. At the start of the breast cancer screening program, the recall rate in the Netherlands was lower than $1 \%$, while typical international recall rates were between 4 and $10 \% .{ }^{11}$ The Dutch optimization study concluded that lowering the recall threshold would increase the breast cancer detection rate. ${ }^{12}$ These recommendations, the routine use of two-view mammography and the introduction of digital mammography screening resulted in more than a doubling of the recall rate (Table 1.1). ${ }^{9}$ These recall rates are still low when compared to the European guidelines, which advice a recall rate of less than $5 \%$ for initial screens and a recall rate of less than $3 \%$ for subsequent screens. ${ }^{13}$

\section{Cancer detection rate}

The increased recall rates from 1998 onwards and the introduction of digital screening mammography in 2004-2010 have increased the number of cancers detected per 1000 screened women (true positives). Among these true positives, the proportion of DCIS (ductal carcinoma in situ) increased markedly from $13-15 \%$ before the introduction of digital screening to over $20 \%$ in $2012 .^{9}$

\section{False positive recall}

False positive results are a significant drawback of screening, without a compensatory benefit for the affected subgroup of participants. During the last decade, the false positive recall rate (FPR) in the Netherlands has risen from $0.5 \%$ in the nineties to $1.7 \%$ in 2012. ${ }^{9}$ That rise is the downside of a successful policy that has increased the cancer detection rate. Despite this increase, the FPR in the Netherlands is still low compared to the United States and other European countries. ${ }^{14,15}$ For a woman living in the Netherlands and attending all thirteen rounds of screening, the cumulative risk of receiving a false positive recall is estimated to be $16 \% .{ }^{16}$ This risk will probably increase further due to the introduction of digital screening.

False positive screening results cause anxiety and distress among the affected women. ${ }^{17,18}$ It is a question of debate whether or not a (repeated) false positive recall rate can influence screening outcome. ${ }^{18-24}$ This is a subject of study in the current thesis. 


\section{Positive predictive value}

The increased recall rates and the introduction of digital screening have increased the number of screen detected cancers invasive and in situ cancers (true positives). However, it has also led to a proportionately much larger increase in false-positive recalls (Figure 1.4). As a result, the positive predictive value (PPV) of recall decreased from $48.4 \%$ in 1998 to $28.0 \%$ in $2012 .^{9}$

Figure 1.4 True positive and false-positive screen results per 1000 screened, 1998-2012 ${ }^{9}$

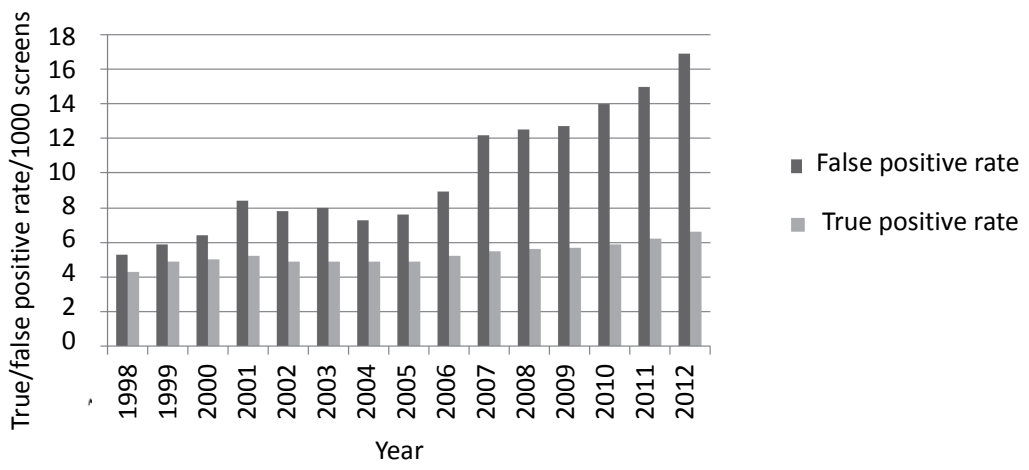

\section{Interval cancer rate}

Interval cancers are breast cancers diagnosed within two years after a negative screen (i.e., no recommendation for recall), prior to the next scheduled biennial screening examination. Interval cancers may either be radiologically occult (true interval cancers) or be missed at previous screening mammography.

The occurrence of interval cancers in women undergoing screening mammography is inevitable. It is important to diminish the amount of interval cancers, as a delay in diagnosis can lead to a more advanced stage of the disease at diagnosis and results in a worse prognosis and survival than screen detected cancers. ${ }^{25,26}$

Despite an increase in cancer detection rate from 1998 onwards, the interval cancer rate has remained the same for many years (Figure 1.5). Before 2004, this might be due to an uncertainty about the reliability of documented interval cancer rates. Recently, a national linkage was established between the Dutch nation-wide screening database and the Dutch Cancer Registry database. This information is now available for the screening period 2004-2009. However, even after 2004 a decline in the interval cancer rate did not occur. ${ }^{9}$ Further, the introduction of digital screening did not influence the occurrence of interval cancers, although interval cancers were more likely to be true interval cancers at digital screening, when compared to screen film mammography $(65.3 \%$ versus $47.1 \%){ }^{27}$ These findings are probably due to an increase in background incidence of breast cancer. ${ }^{9}$ 
Figure 1.5 Screen detected and interval cancers per 1000 screened, 1998-2012 ${ }^{9}$

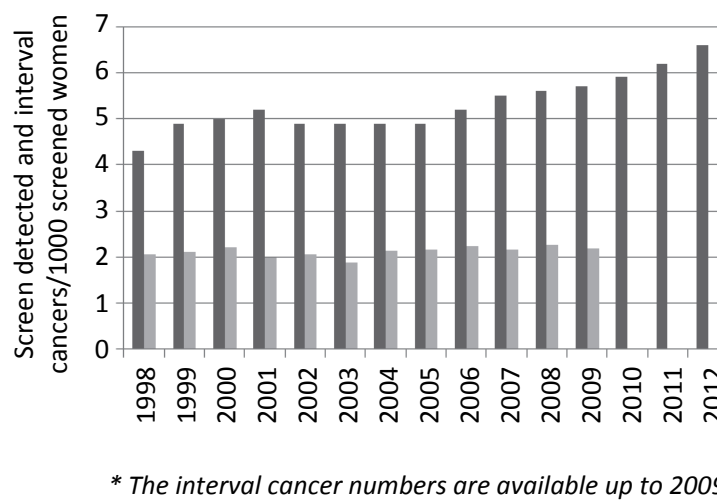

- Screen detected cancers

- Interval cancers

\section{Screening sensitivity}

Screening sensitivity measures the proportion of breast cancers that are detected within the breast screening program (screen detected cancers and interval cancers). Sensitivity can be determined in two ways: 1) based on all interval cancers (program sensitivity) and 2) based on missed interval cancers only (mammographic sensitivity). Program sensitivity is an important indicator for the evaluation of screening programs. Although the interval cancer rate remained the same for many years, the increasing detection of breast cancers by the Dutch screening program is boosting the program sensitivity. This is partly due to an increased detection of DCIS by digital screening. However, the increase in sensitivity of invasive cancers alone from 2007 to 2009 was also higher compared to previous years. ${ }^{9}$

\section{Screening specificity}

The program specificity is and has always been high compared to other countries. About $99 \%$ of the women screened, who did not have breast cancer, received no recommendation for recall. Program specificity is primarily dependent on the recall rate, i.e. the higher the recall rate the lower the specificity. Despite the increase in recall rate seen in the Dutch screening program over the past years, and therewith a small decrease in program specificity (Table 1.1), the specificity is still relatively high in international terms. ${ }^{9}$

\section{Factors influencing screening outcome}

Screening outcome can be influenced by a number of parameters including patient related (e.g. breast density, previous breast surgery, breast cancer prevalence), imaging related (e.g. image quality, number of views, screen film of digital mammography) and 
screening situation related parameters (e.g. reading strategy, radiologist performance, screening interval).

The three aspects of the screening program that will be discussed in this thesis are: the transition from screen film to digital mammography, reading strategies and radiologist performance. These subjects will be introduced in further detail below.

\section{The transition from screen-film to digital mammography}

Digital screening mammography has been available for several years now and has replaced screen-film mammography in most screening programs worldwide. In the Netherlands, all screening organizations switched to digital mammography in the period 2004-2010. The introduction of digital mammography in the Netherlands was accompanied by higher cancer detection rates at the expense of higher recall rates, false positive rates and an increased detection of DCIS. ${ }^{9,28-30}$

To facilitate comparison of subsequent digital screens with prior screen-film mammograms during the transition, the most recent screen-film mammograms were digitized by fusion equipment designed for mammography. In this thesis we will evaluate if the availability of prior hard copy screen-films could reduce the number of patients with a repeated false positive recall rate for the same mammographic lesion.

\section{Reading strategies}

Assessment of screening mammograms can be performed in several ways, e.g. by single radiologist reading, single reading with computer aided detection (CAD), or double reading. Double reading by two specialized breast screening radiologists is considered to be the standard of reference in Europe. ${ }^{13}$ This reading strategy significantly increases the cancer detection rate when compared to single reading at the expense of a small increase in recall rate. ${ }^{31-33}$ Assessment by CAD $^{34}$ or screening mammography technologists, ${ }^{35,36}$ in addition to radiologist single reading, may be considered when radiologist double reading is not feasible due to a shortage of trained and experienced radiologists.

Double reading can be performed in a blinded $\left(2^{\text {nd }}\right.$ reader is not informed about the $1^{\text {st }}$ reader's decision) or non-blinded fashion $\left(2^{\text {nd }}\right.$ reader is informed about the $1^{\text {st }}$ reader's decision). The Dutch Health Council of the Netherlands advises the use of blinded double reading. ${ }^{1}$ However, very few studies report on the advantages and disadvantages of blinded double reading as compared to non-blinded double reading, ${ }^{37}$ and these two reading strategies were never compared in a screening setting. In the era of screen-film mammography, mammograms were usually read in a non-blinded fashion. With the introduction of digital screening it became technically feasible to perform blinded double reading as an alternative to non-blinded double reading.

Double reading is accompanied by discrepant readings. There are different ways to deal with these discrepant readings. First, women with a discrepant reading may always be recalled for further evaluation. Alternatively, the two radiologists may try to reach 
consensus in case of a discrepant reading, or arbitration by a third radiologist can be performed. In case of a discrepant reading, one of these alternative reading strategies may lower the recall rate and amount of false positive recalls, but possibly at the expense of a lower detection rate. A few studies have evaluated the effect of consensus meetings $^{38}$ and arbitration ${ }^{39-41}$ on screening outcome at screen film mammography (SFM) at non-blinded double reading. These studies showed that arbitration and consensus meetings resulted in reduced recall rates, at the expense of a small decrease in cancer detection rate. However, the effect of arbitration was never determined in a setting with blinded double reading or in the era of digital screening.

In this thesis we will prospectively evaluate the screening outcomes of blinded and non-blinded reading in a population based study. Further, we will explore how to cope best with discrepant readings i.e., recall of all discrepant readings; arbitration of all discrepant readings by a third reader; or only arbitration of discrepant BI-RADS 0 recalls by a third reader.

\section{Radiologist performance}

Radiologists differ in their ability to interpret screening mammograms, known as interobserver variability. ${ }^{42-45}$ The source of radiologist variability has not been fully explained yet. There are conflicting reports whether or not a greater volume of mammograms read per year, or experience at interpreting mammograms, is associated with better performance. ${ }^{11,46-49} \mathrm{~A}$ positive relation has been reported between cancer detection rate and radiologists performing more diagnostic work-up ${ }^{47}$ and radiologists with fellowship training in breast cancer screening. ${ }^{50}$

As mentioned above, double reading of screening mammograms is the standard of reference in Europe. It is not known whether or not variability in the ability to interpret screening mammograms per pair of screening radiologists persist at double reading. This is a subject of the current thesis. 


\section{Outline of this thesis}

The objective of this thesis is to explore several factors of the breast cancer screening program in the Netherlands with the aim to improve the effectiveness of the Dutch breast cancer program.

Chapter 2 evaluates the re-attendance rate at screening mammography after a single or repeated false positive recall and determines the effect of the transition to digital screening on the proportion and screening outcome of women recalled twice for the same mammographic abnormality.

Chapter 3 determines the variations in screening performances among unique pairs of screening radiologists at non-blinded double reading of screening mammograms.

Chapter 4 shows the results of a prospective study evaluating the results of blinded versus non-blinded double reading. Chapter 4.1 shows the general screening outcomes of the two reading strategies. Chapter $\mathbf{4 . 2}$ describes the utilization of breast imaging and biopsy techniques at the two strategies. Chapter 4.3 shows the role of arbitration of all discrepant readings by a third reader and chapter 4.4 evaluates the characteristics of low suspicion lesions (BI-RADS 0 ) and determines the potential effect of arbitration of discrepant BI-RADS 0 readings by a third reader on screening outcome.

In chapter $\mathbf{5}$ we discuss the main results and the future perspectives of the studies in this thesis and we propose certain strategies that may improve the effectiveness of population based breast cancer screening. 


\section{References}

1 Population screening for breast cancer: expectations and developments. Heath Council of the Netherlands 2014.

2 Jemal A, Bray F, Center MM, Ferlay J, Ward E, Forman D. Global cancer statistics. CA Cancer J Clin 2011; 61:69-90.

$3 \quad$ IKNL cijfers over kanker. Available via www.cijfersoverkanker.nl.

4 Soerjomataram I, Pukkala E, Brenner H, Coebergh JW. On the avoidability of breast cancer in industrialized societies: older mean age at first birth as an indicator of excess breast cancer risk. Breast Cancer Res Treat 2008;111:297-302.

5 Jones AL. Reduction in mortality from breast cancer. BMJ 2005;330:205-206.

6 Berry DA, Cronin KA, Plevritis SK et al. Effect of screening and adjuvant therapy on mortality from breast cancer. N Engl J Med 2005;353:1784-1792.

7 Vanel D. The American College of Radiology (ACR) Breast Imaging and Reporting Data System (BI-RADS): a step towards a universal radiological language? European Journal of Radiology 2007;61:183.

8 Timmers JM, van Doorne-Nagtegaal HJ, Zonderland HM et al. The Breast Imaging Reporting and Data System (BI-RADS) in the Dutch breast cancer screening programme: its role as an assessment and stratification tool. Eur Radiol 22012;2:1717-1723.

9 National Evaluation Team for Breast Cancer Screening in Netherlands Study G. National evaluation of breast cancer screening the Netherlands 1990-2011/2012. In: Erasmus MC R, (ed). NETB 2014.

10 National Institute of Public Health and the Enviroment (RIVM). Available via www.rivm.nl

11 Smith-Bindman R, Chu PW, Miglioretti DL et al. Comparison of screening mammography in the United States and the United kingdom. JAMA 2003;290:2129-2137.

12 Otten JD, Karssemeijer N, Hendriks JH et al. Effect of recall rate on earlier screen detection of breast cancers based on the Dutch performance indicators. J Natl Cancer Inst 2005;97:748-754.

13 Perry N, Broeders M, de Wolf C, Tornberg S, Holland R, von Karsa L. European guidelines for quality assurance in breast cancer screening and diagnosis. Fourth edition--summary document. Ann Oncol 2008;19:614-622.

14 Fletcher SW, Elmore JG. False-positive mammograms--can the USA learn from Europe? Lancet 2005;365:7-8.

15 Roman M, Hubbard RA, Sebuodegard S, Miglioretti DL, Castells X, Hofvind S. The cumulative risk of false-positive results in the Norwegian Breast Cancer Screening Program: updated results. Cancer 2013;119:3952-3958.

16 Otten JD, Fracheboud J, den Heeten GJ et al. Likelihood of early detection of breast cancer in relation to false-positive risk in life-time mammographic screening: population-based cohort study. Ann Oncol 2013;24:2501-2506.

17 van der Steeg AF, Keyzer-Dekker CM, De Vries J, Roukema JA. Effect of abnormal screening mammogram on quality of life. Br J Surg 2011;98:537-542.

18 Brewer NT, Salz T, Lillie SE. Systematic review: the long-term effects of false-positive mammograms. Ann Intern Med 2007;146:502-510.

19 Setz-Pels W, Duijm LE, Coebergh JW, Rutten M, Nederend J, Voogd AC. Re-attendance after falsepositive screening mammography: a population-based study in the Netherlands. Br J Cancer 2013; 109:2044-2050.

20 Seigneurin A, Exbrayat C, Labarere J, Delafosse P, Poncet F, Colonna M. Association of diagnostic workup with subsequent attendance in a breast cancer screening program for false-positive cases. Breast Cancer Res Treat 2011;127:221-228.

21 Roman R, Sala M, De La Vega M et al. Effect of false-positives and women's characteristics on long-term adherence to breast cancer screening. Breast Cancer Res Treat 2011;130:543-552.

22 Alamo-Junquera D, Murta-Nascimento C, Macia F et al. Effect of false-positive results on reattendance at breast cancer screening programmes in Spain. Eur J Public Health 2012;22:404-408.

23 Andersen SB, Vejborg I, von Euler-Chelpin M. Participation behaviour following a false positive test in the Copenhagen mammography screening programme. Acta Oncol 2008;47:550-555.

24 Johns LE, Moss SM. False-positive results in the randomized controlled trial of mammographic screening from age 40 ("Age" trial). Cancer Epidemiol Biomarkers Prev 2010;19:2758-2764. 
25 Fong Y, Evans J, Brook D, Kenkre J, Jarvis P, Gower Thomas K. The incidence and 10-year survival of interval breast cancers in Wales. Clin Radiol 2014;69:e168-172.

26 Richards MA, Smith P, Ramirez AJ, Fentiman IS, Rubens RD. The influence on survival of delay in the presentation and treatment of symptomatic breast cancer. Br J Cancer 1999;79:858-864.

27 Nederend J, Duijm LE, Louwman MW et al. Impact of the transition from screen-film to digital screening mammography on interval cancer characteristics and treatment - a population based study from the Netherlands. Eur J Cancer 2014;50:31-39.

28 Nederend J, Duijm LE, Louwman MW, Groenewoud JH, Donkers-van Rossum AB, Voogd AC. Impact of transition from analog screening mammography to digital screening mammography on screening outcome in The Netherlands: a population-based study. Ann Oncol 2012;23:3098-3103.

29 Bluekens AM, Karssemeijer N, Beijerinck D et al. Consequences of digital mammography in populationbased breast cancer screening: initial changes and long-term impact on referral rates. Eur Radiol 2010;20:2067-2073.

30 van Luijt PA, Fracheboud J, Heijnsdijk EA, den Heeten GJ, de Koning HJ. Nation-wide data on screening performance during the transition to digital mammography: Observations in 6 million screens. Eur J Cancer 2013;49:3517-3525.

31 Harvey SC, Geller B, Oppenheimer RG, Pinet M, Riddell L, Garra B. Increase in cancer detection and recall rates with independent double interpretation of screening mammography. AJR Am J Roentgenol 2003;180:1461-1467.

32 Thurfjell EL, Lernevall KA, Taube AA. Benefit of independent double reading in a population-based mammography screening program. Radiology 1994;191:241-244.

33 Brown J, Bryan S, Warren R. Mammography screening: an incremental cost effectiveness analysis of double versus single reading of mammograms. BMJ 1996;312:809-812.

34 Gilbert FJ, Astley SM, Gillan MG et al. Single reading with computer-aided detection for screening mammography. N Engl J Med 2008;359:1675-1684.

35 Tonita JM, Hillis JP, Lim CH. Medical radiologic technologist review: effects on a population-based breast cancer screening program. Radiology 1999;211:529-533.

36 Duijm LE, Groenewoud JH, Fracheboud J, de Koning HJ. Additional double reading of screening mammograms by radiologic technologists: impact on screening performance parameters. J Natl Cancer Inst 2007;99:1162-1170.

37 Groenewoud JH, Otten JD, Fracheboud J et al. Cost-effectiveness of different reading and referral strategies in mammography screening in the Netherlands. Breast Cancer Res Treat 2007;102:211-218.

38 Shaw CM, Flanagan FL, Fenlon HM, McNicholas MM. Consensus review of discordant findings maximizes cancer detection rate in double-reader screening mammography: Irish National Breast Screening Program experience. Radiology 2009;250:354-362.

39 Duijm LE, Groenewoud JH, Hendriks JH, de Koning HJ. Independent double reading of screening mammograms in The Netherlands: effect of arbitration following reader disagreements. Radiology 2004;231:564-570.

40 Ciatto S, Ambrogetti D, Risso G et al. The role of arbitration of discordant reports at double reading of screening mammograms. J Med Screen 2005;12:125-127.

41 Caumo F, Brunelli S, Tosi E et al. On the role of arbitration of discordant double readings of screening mammography: experience from two Italian programmes. Radiol Med 2011;116:84-91.

42 Elmore JG, Wells CK, Lee $\mathrm{CH}$, Howard DH, Feinstein AR. Variability in radiologists' interpretations of mammograms. N Engl J Med 1994;331:1493-1499.

43 Beam C, Sullivan D. Variability in mammogram interpretation. Adm Radiol J 1996;15:47, 49-50, 52.

44 Duijm LE, Louwman MW, Groenewoud JH, van de Poll-Franse LV, Fracheboud J, Coebergh JW. Interobserver variability in mammography screening and effect of type and number of readers on screening outcome. Br J Cancer 2009;100:901-907.

45 Skaane $\mathrm{P}$, Diekmann F, Balleyguier $\mathrm{C}$ et al. Observer variability in screen-film mammography versus fullfield digital mammography with soft-copy reading. Eur Radiol 2008;18:1134-1143.

46 Theberge I, Chang SL, Vandal N et al. Radiologist interpretive volume and breast cancer screening accuracy in a Canadian organized screening program. J Natl Cancer Inst 2014;106:djt461.

47 Buist DS, Anderson ML, Haneuse SJ et al. Influence of annual interpretive volume on screening mammography performance in the United States. Radiology 2011;259:72-84. 
48 Barlow WE, Chi C, Carney PA et al. Accuracy of screening mammography interpretation by characteristics of radiologists. J Natl Cancer Inst 2004;96:1840-1850.

49 Miglioretti DL, Gard CC, Carney PA et al. When Radiologists Perform Best: The Learning Curve in Screening Mammogram Interpretation. Radiology 2009;253:632-640.

50 Elmore JG, Jackson SL, Abraham L et al. Variability in interpretive performance at screening mammography and radiologists' characteristics associated with accuracy. Radiology 2009;253:641-651. 


\section{Chapter 2}

Re-attendance at biennial screening mammography following a repeated false positive recall

Klompenhouwer EG, Duijm LEM, Voogd AC, Heeten GJ, Strobbe LJ, Louwman MW, Coebergh JW, Venderink D, Broeders MJM

Breast Cancer Res Treat 2014;145:429-37 


\section{Abstract}

\section{Objectives}

To determine the re-attendance rate at screening mammography after a single or a repeated false positive recall and to assess the effects of transition from screen-film mammography (SFM) to full-field digital mammography (FFDM) on screening outcome in women recalled twice for the same mammographic abnormality.

\section{Methods}

The study population consisted of a consecutive series of 302,912 SFM and 90,288 FFDM screens. During a two years follow-up period (until the next biennial screen) we collected the breast imaging reports and biopsy results of all recalled women.

\section{Results}

Re-attendance at biennial screening mammography was 93.2\% (95\%Cl- 93.1\%-93.3\%) for women with a negative screen (i.e., no recall at screening mammography), $65.4 \%$ ( $95 \% \mathrm{Cl} 64.0 \%-66.8 \%$ ) for women recalled once, $56.7 \%(95 \% \mathrm{Cl} 47.1 \%-66.4 \%)$ for women recalled twice but for different lesions and $44.3 \%(95 \% \mathrm{Cl} 31.4 \%-57.1 \%)$ for women recalled twice for the same lesion. FFDM recalls comprised a significantly larger proportion of women who had been recalled twice for the same lesion ( $1.9 \%$ of recalls ( 52 women) at FFDM versus $0.9 \%$ of recalls ( 37 women) at SFM, $P<0.001)$ and the positive predictive value of these recalls (PPV) was significantly lower at FFDM ( $15.4 \%$ versus $35.1 \%, P=0.03$ ). At review, 20 of 52 women (39.5\%, all with benign outcome) would not have been recalled for a second time at FFDM if the previous hard copy SFM screen had been available for comparison.

\section{Conclusion}

A repeated false positive recall for the same lesion significantly lowered the probability of screening re-attendance. The first round of FFDM significantly increased the proportion of women recalled twice for the same lesion, with a significantly lower PPV of these lesions. Almost $40 \%$ of repeatedly recalled women would not have been recalled the second time if the previous hard copy SFM screen had been available for comparison at the time of FFDM. 


\section{Introduction}

In the last two decades, many Western countries have implemented screening mammography programs with the aim to reduce mortality from breast cancer through early detection. ${ }^{1}$

Screening programs seek to find an optimal balance between recall rate and positive predictive values. Recall of lesions that turn out to be benign should be avoided as much as possible in order to minimize anxiety among the screened population and to minimize unnecessary work up costs. ${ }^{2-4}$

The effect of a false positive recall on re-attendance at subsequent screening mammography remains a question of debate. A meta-analysis published in 2007 reported no significant relationship between false positive mammograms and return for routine screening among European women, a decreased likelihood of re-attendance among Canadian women and an increased re-attendance rate among women who experienced a false positive mammogram in the United States. ${ }^{5}$ Recently, four European studies showed a lower screening re-attendance rate after a false positive screening mammogram. ${ }^{6-9}$ In contrast, the Copenhagen mammography screening program $^{10}$ and the UK Age Trial ${ }^{11}$ found no association of a false positive screen with screening re-attendance. Taking into account the possible negative effects of a false positive screening result, it is especially important to minimize the number of women who experience a recurrent false positive recall for the same lesion.

Studies on re-attendance after a false positive recall have mainly focused on women assessed by screen film mammography. Full-field digital mammography (FFDM) has been available for several years now and has replaced screen-film mammography (SFM) in most screening programs. In the Netherlands, the introduction of FFDM was accompanied by higher cancer detection rates at the expense of higher recall rates and a decreased positive predictive value of recall (PPV). ${ }^{12-14}$ The effect of the transition from SFM to FFDM screening mammography on the number of women recalled twice for the same lesion has not yet been explored and it is unknown if any measures can be taken in order to reduce repeated false positive recalls at FFDM.

The purpose of the current study was to determine the re-attendance rate at biennial screening mammography after a single or repeated false positive recall and to determine the effect of the transition to FFDM on the proportion and screening outcome of women recalled twice for the same lesion. We also assessed the possible influence of comparison with scanned in priors instead of hard copy priors at FFDM on the proportion of women recalled twice for the same mammographic abnormality. 


\section{Materials and methods}

\section{Study population}

We included a consecutive series of 302,912 SFM screens and 90,288 FFDM screens. Of these, 90,268 were initial screens and 302,932 were subsequent screens. The screens were obtained at three specialized screening units in a southern biennial screening mammography region of the Netherlands (BOZ, BevolkingsOnderzoekZuid). The SFM and FFDM screens were performed, respectively between January 2000-April 2010 and May 2009-July 2011.

Women participating in the nationwide Dutch screening program are asked to give permission for the use of their data for scientific purposes. Three women screened at our units did not give this permission and they were excluded from analysis. Ethical approval for this study was waived by the Central Committee on Research Involving Human Subjects (CCMO) in The Hague, The Netherlands.

\section{Screening procedure and recall}

Details of the nationwide breast cancer screening program have been described previously. ${ }^{15,16}$ In brief, this program provides free biennial screening mammography for women aged 50-75 years since 1990. It is organized by a system of personal invitations with a program compliance rate of over $80 \%$.

Screen-film mammograms at the southern screening mammography region of the Netherlands were obtained with commercially available units (Performa, Oldelft, Tuusula, Finland). All digital mammograms were acquired with a LoradSelenia FFDM system (Hologiclnc, Danbury, CT), with a $70 \mu \mathrm{m}$ pixel size and a 232x286 mm field of view. The mammographic examinations were obtained by specialized screening mammography technologists and double read by a team of 12 certified screening radiologists. Each radiologist evaluated at least 3,000 screening mammograms yearly (with a mean of 6,000 ). Prior screening mammograms were always available for comparison at the time of a subsequent screening round at SFM. To facilitate comparison of subsequent FFDM screens with prior SFM screens, the most recent screen-film mammograms were digitized by fusion equipments designed for mammography (DigitalNow;R2/Hologic).

Women with normal or benign mammographic findings, or with a non-specific minimal sign, ${ }^{17}$ were not recalled. Women with suspicious malignant findings were recalled and evaluated at a dedicated breast hospital. For each recall, the screening radiologists classified the abnormal mammographic findings according to the following categories: suspicious mass, suspicious microcalcifications, mass in combination with microcalcifications, architectural distortion, asymmetry or other abnormality. In addition, each screen was classified according to screening BI-RADS in case of FFDM screening mammography. 


\section{Diagnostic work-up and follow-up of recalled women}

A total of 15 regional and university hospitals were involved in the assessment of screen-positive women. After physical examination by the surgeon, additional mammographic views were obtained if necessary. At diagnostic workup, radiologists classified the radiological findings according to the Breast Imaging Reporting and Data System, BI-RADS. ${ }^{18}$ Dependent on the findings at physical examination and mammography, the diagnostic workup protocols and hospital facilities available, further diagnostic evaluation could include breast ultrasonography, magnetic resonance mammography, percutaneous fine-needle aspiration biopsy (FNAB), core needle biopsy (CNB) or open excision biopsy. BI-RADS 4 or 5 lesions were routinely biopsied, whereas BI-RADS 3 lesions were either biopsied or followed-up.

During a follow-up period of at least two years (until the next biennial screening round), we collected data on diagnostic imaging procedures, clinical data, biopsy specimen and surgical procedures of all recalled women. Breast cancers were divided into ductal carcinoma in situ (DCIS) and invasive cancers; lobular carcinoma in situ was considered as non-malignant. Data on TNM (tumor-node-metastases) classification ${ }^{19}$ were collected. Advanced cancers were defined as invasive cancers with TNM stage IIA or higher, i.e. tumor size exceeding $20 \mathrm{~mm}$ (T2) and/or the presence of axillary metastasis. ${ }^{20}$ Sentinel nodes were classified negative if they harbored isolated tumor cells or sub-micrometastases $\left(<0.2 \mathrm{~mm}, \mathrm{NO}_{(\text {itc })}\right)$ and were considered positive if they contained micrometastases $\left(0.2-2 \mathrm{~mm}, \mathrm{~N} 1_{(\mathrm{mi})}\right)$ or macrometastases ( $>2 \mathrm{~mm}, \mathrm{N1}-3$ ).

\section{Identification and review of women with a repeated recall}

All data were stored in an Excel database (Excel; Microsoft, Redmond, WA, USA). The database was used to identify women who had been recalled twice. One screening radiologist (L.E.M.D, with 16 years of experience in breast imaging) determined whether or not the second recall comprised the same lesion. Subsequently, in case of repeated recall for the same lesion at FFDM, the original screening couple reassessed the screen for which a woman had been recalled. At that occasion, the hard copy screen-film mammogram was available in addition to the digitized screen-film mammogram. The radiologists determined whether comparison with the hard copy screen would have altered their decision for second recall. At this review, the radiologists were blinded for the pathologic outcome of screening.

\section{Determination of re-attendance at biennial screening mammography}

Linkage of the $\mathrm{BOZ}$ database and the national screening database to the regional register of Death (Gemeentelijke Basisadministratie Persoonsgegevens) enabled us to identify women who had died after attending screening mammography and identify women who had received a subsequent screen in another screening region. In order to determine exactly those women eligible for rescreening after a previous false positive screen (i.e., no diagnosis of breast cancer at workup), we identified those women who 
had died and those women who had turned 76 years before the next screening round. These women, as well as women diagnosed with an interval cancer, were no longer eligible for screening and were therefore defined as the 'non-target' group. An interval cancer was defined as a cancer diagnosed before the next (biennial) screening round after a previous negative screen. Procedures for the identification of interval cancers have been described previously. ${ }^{21}$

\section{Statistical analysis}

Descriptive statistics were performed using Statistical Package for Social Science 17.0 (SPSS Inc. Chicago, IL). A Fisher's exact test or chi-squared test was used to test differences between SFM and FFDM screens. A t-test was used to determine the $95 \% \mathrm{Cl}$ of the re-attendance rates. A $P$-value of less than .05 was considered to indicate statistical significance.

\section{Results}

\section{Cohort results}

The cohort characteristics of the 302,912 SFM screens and 90,288 FFDM screens are shown in Table 2.1 and Figure 2.1. The recall rate and the cancer detection rate (per 1,000 screens) were significantly higher at FFDM than at SFM (3.1\% versus $1.4 \%$ $(P<0.001)$ and 6.8 versus $5.1(P<0.001)$ respectively), at the expense of a lower positive predictive value of recall at FFDM (22.0\% versus $37.3 \%, P<0.001)$. Compared with SFM screening, a significantly larger proportion of women recalled at FFDM screening showed suspicious microcalcifications $(28.9 \%$ versus $17.6 \%, P<0.001)$, whereas a smaller percentage of women were recalled for evaluation of a suspicious mass $(56.9 \%$ versus $69.9 \%, P<0.001)$. 
Table 2.1 Overall screening outcome at screen-film and full-field digital mammography

\begin{tabular}{|c|c|c|c|}
\hline & $\begin{array}{c}\text { Screen-film } \\
\text { mammography* }\end{array}$ & $\begin{array}{l}\text { Full-field digital } \\
\text { mammography* }\end{array}$ & P-value \\
\hline Screens, No & 302,912 & 90,288 & \\
\hline Initial screens, $\mathrm{n}(\%)$ & $34,897(11.5)$ & $10,063(11.1)$ & \\
\hline Subsequent screens, n (\%) & $268,015(88.5)$ & 80,225 (88.9) & \\
\hline Recall, No (\%) & $4,140(1.4)$ & $2,782(3.1)$ & $<0.001$ \\
\hline Reason of recall, No (\%) & & & $<0.001$ \\
\hline Suspicious mass & $2,893(69.9)$ & $1,582(56.9)$ & \\
\hline Suspicious microcalcifications & $9(17.6)$ & 804 (28.9) & \\
\hline Suspicious mass with microcalcifications & $287(6.9)$ & $186(6.7)$ & \\
\hline Architectural distortion & $108(2.6)$ & $52(1.9)$ & \\
\hline Asymmetry & $119(2.9)$ & $153(5.5)$ & \\
\hline Other mammographic abnormality & $4(0.1)$ & $5(0.2)$ & \\
\hline Screen detected breast cancer, No & 1,544 & 613 & \\
\hline Positive predictive value of recall, $\%$ & 37.3 & 22.0 & $<0.001$ \\
\hline Cancer detection rate per 1,000 screens & 5.1 & 6.8 & $<0.001$ \\
\hline
\end{tabular}

Figure 2.1 Overall screening mammography re-attendance two years following a negative screen

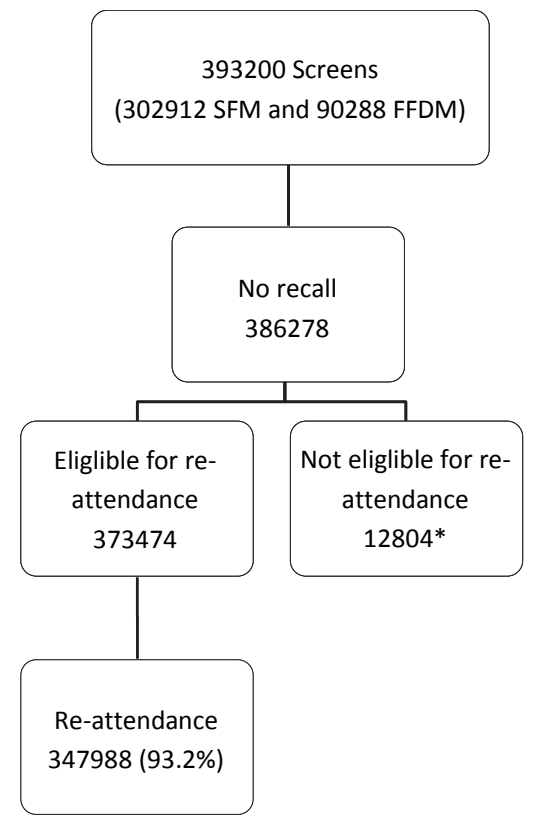

SFM Screen-film mammography; FFDM Full-field digital mammography. *Including 2162 deceased women, 779 women diagnosed with interval breast cancer, 9863 women aged 76 years at the time of the next screening round 


\section{Re-attendance at biennial screening mammography}

A total of 474,879 invitations were send during the study period, resulting in 393,200 screens, with an overall attendance rate of $82.8 \%$. The screening radiologists had mentioned no indication for recall in 386,278 screens. A total of 12,804 of these women were not eligible for re-attendance (non-target group): 2,162 had died, 779 women had been diagnosed with an interval cancer and 9,863 women had turned 76 years before the next screening round. There were 25,486 women in the target group, with no indication for recall, who did not re-attend the subsequent screening round. Therefore, the re-attendance rate after a negative screen was $93.2 \%(95 \% \mathrm{Cl}-93.1 \%-93.3 \%)$ (Figure 2.1). Re-attendance at biennial screening mammography was $65.4 \%(95 \% \mathrm{Cl}$ $64.0 \%-66.8 \%)$ for women recalled once, $56.7 \%(95 \% \mathrm{Cl} 47.1 \%-66.4 \%)$ for women recalled twice but for different lesions and $44.3 \%(95 \% \mathrm{Cl} 31.4 \%-57.1 \%)$ for women recalled twice for the same lesion (Figure 2.2). Finally, $52.1 \%(95 \% \mathrm{Cl} 44.4 \%-59.8 \%)$ of all women who had experienced a repeated false positive recall, whether or not for the same mammographic abnormality, re-attended the next biennial screening mammography.

Figure 2.2 Screening mammography re-attendance two years following a single or a repeated false positive recall

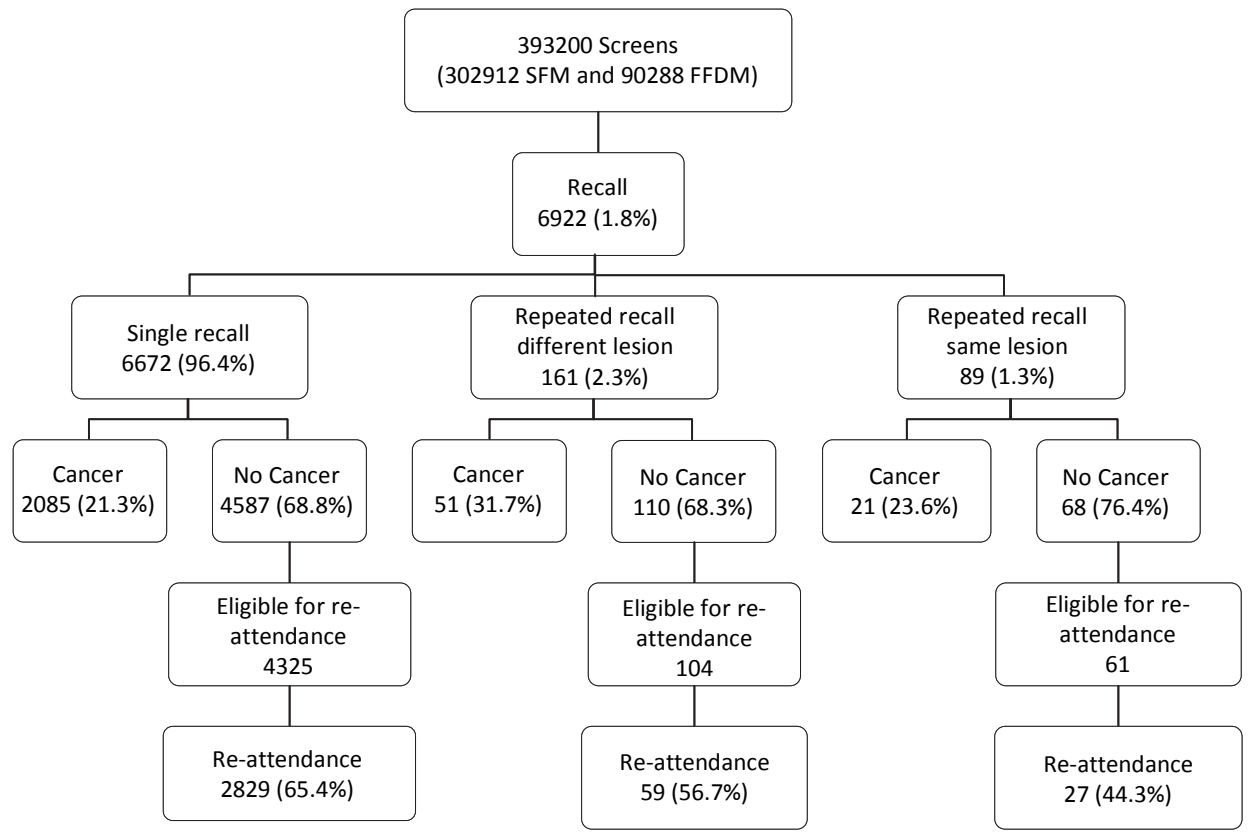

SFM Screen-film mammography; FFDM Full-field digital mammography 


\section{Women recalled twice for the same lesion}

Out of 4,140 recalled women at SFM, 37 ( $0.9 \%$ of recalls) had been recalled twice for the same lesion, of which 13 proved to be malignant ( $0.8 \%$ of screen detected cancers). Thus, the positive predictive value of second recall was $35.1 \%$. Breast cancer in these 13 women was diagnosed one or several screening rounds after the initial recall and the mean delay in cancer diagnosis was 44 months (range, 24-97 months).

Out of 2,782 women recalled at FFDM, 52 (1.9\% of recalls) had been recalled twice for the same lesion $\left(1^{\text {st }}\right.$ recall at SFM, $2^{\text {nd }}$ recall at FFDM), of which 8 ( $1.3 \%$ of screen detected cancers) proved to be malignant. Thus the positive predictive value of second recall was $15.4 \%$, which was significantly lower than the $35.1 \%$ at SFM $(P=0.03)$ (Table 2.2, Figure 2.3). The mean delay in breast cancer diagnosis in these 8 women was 44 months (range, 26-73 months).

At FFDM, a significantly larger proportion of recalled women comprised repeated recall for the same lesion ( $1.9 \%$ versus $0.9 \%$, or 0.58 versus 0.12 per 1,000 screens, $P<0.001)$.

When comparing SFM to FFDM, no statistically significant differences were observed in the reason of recall: the distribution of mammographic abnormalities among repeatedly recalled women were comparable for SFM and FFDM, with half of the lesions characterized as a suspicious mass (Table 2.2).

Table 2.2 Screening and outcome characteristics of women recalled twice for the same lesion at screenfilm mammography and full-field digital mammography

$\begin{array}{lccc}\text { No. of women recalled twice for the same lesion } & \begin{array}{c}\text { Screen-film } \\ \text { mammography* }\end{array} & \begin{array}{c}\text { Full-field digital } \\ \text { Mammography* }\end{array} & \text { P-value } \\ \text { Proportion of total recalls, \% } & 37 & 52 & <0.001 \\ \text { Per 1,000 screens, \%o } & 0.9 & 1.9 & 0.03 \\ \text { No. (\%) of women diagnosed with breast cancer } & 0.12 & 0.58 & 0.32 \\ \text { Percentage of screen detected cancers } & 13(35.1) & 8(15.4) & 0.15 \\ \text { Reason of recall, No (\%) } & 0.8 & 1.3 & \\ \text { Suspicious mass } & & & \\ \text { - Malignant } & 19(51.4) & 6(75.0) & \\ \text { Suspicious microcalcifications } & 9(69.2) & 15(28.8) & 0(0.0) \\ \text { - Malignant } & 16(43.2) & 8(15.4) & 1(12.5) \\ \text { Suspicious mass with microcalcifications } & 3(23.1) & 3(5.8) & \\ \text { - Malignant } & 2(5.4) & 1(12.5) & \\ \text { Architectural distortion } & 0(0.0) & 0(0.0) & \end{array}$

* Screen-film mammography: 1st and 2nd recall at screen-film mammography; full-field digital mammography: 1st recall at screen-film mammography, 2nd recall at full-field digital mammography 
Figure 2.3: Mammography screening outcome at screen-film mammography and full-field digital mammography of patients recalled twice for the same lesion, 2000-2011

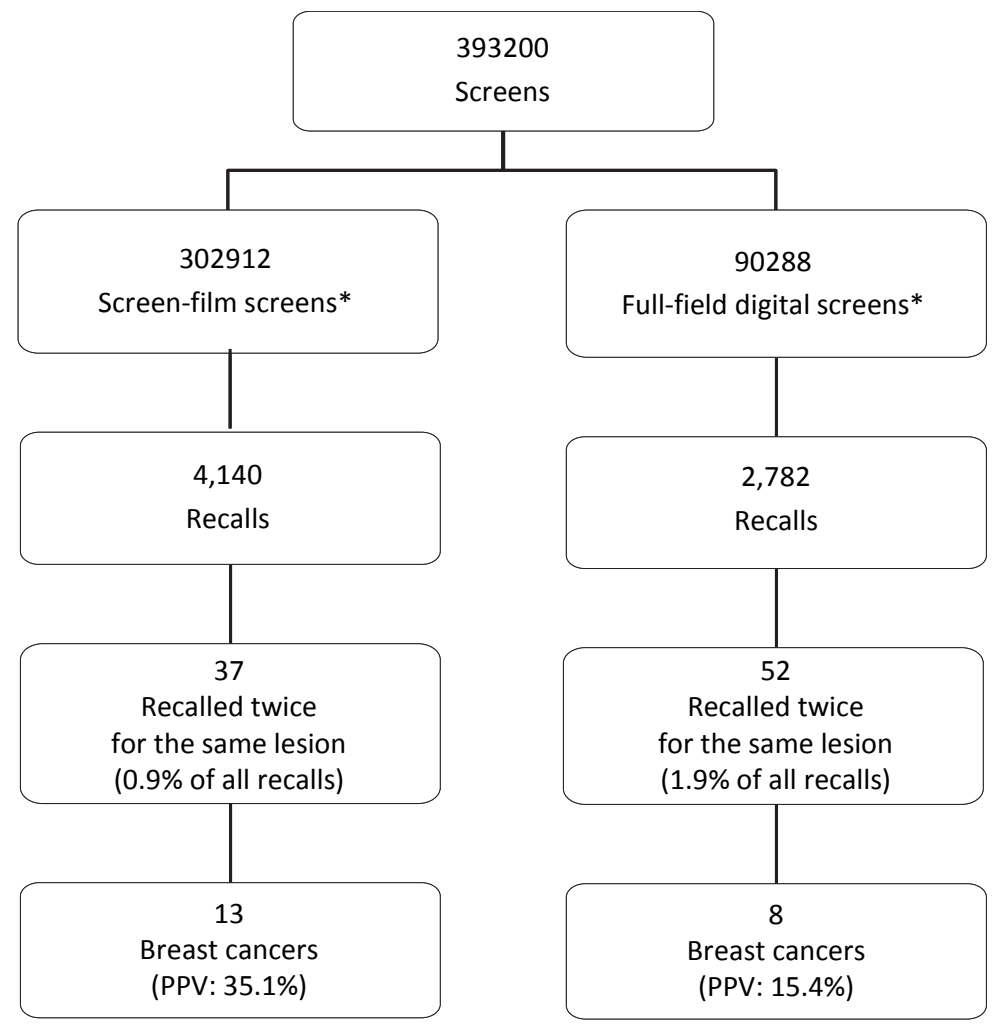

* Screen-film mammography: 1st and 2nd recall at screen-film mammography; Full-field digital mammography: 1st recall at screen-film mammography, 2nd recall at full-field digital mammography. PPV positive predictive value of recall

\section{Women diagnosed with breast cancer at second recall}

At FFDM, none of the 15 patients recalled twice for suspicious microcalcifications was eventually diagnosed with breast cancer, compared to 6 out of 26 patients $(23.1 \%)$ recalled for a suspicious mass $(P=0.07)$. At SFM, 3 out of 16 patients $(18.3 \%)$ recalled twice for suspicious microcalcifications and 9 of the 19 patients $(47.4 \%)$ recalled for a suspicious mass were eventually diagnosed with breast cancer $(P=0.15$, Table 2.2 ).

No significant differences were found between women diagnosed with breast cancer after second recall at SFM or FFDM with respect to the proportion of invasive cancers, size of invasive cancers, axillary lymph node stage or the proportion of advanced cancers (Table 2.3). 
Table 2.3 Tumor characteristics of women recalled twice for the same lesion at screen-film mammography and full-field digital mammography

\begin{tabular}{|c|c|c|c|}
\hline & $\begin{array}{c}\text { Screen-film } \\
\text { Mammography* } \\
\text { (13 breast cancers) }\end{array}$ & $\begin{array}{l}\text { Full-field digital } \\
\text { Mammography* } \\
\text { (8 breast cancers) }\end{array}$ & P-value \\
\hline Tumor type, No (\%) & & & 1.00 \\
\hline Ductal carcinoma in situ & $1(7.7)$ & $1(12.5)$ & \\
\hline Invasive cancer & $12(92.3)$ & $7(87.5)$ & \\
\hline Tumor stage of invasive cancers, No (\%) & & & 0.60 \\
\hline T1a-c & $10(83.3)$ & $5(71.4)$ & \\
\hline $\mathrm{T} 2+$ & $2(16.7)$ & $2(28.6)$ & \\
\hline $\begin{array}{l}\text { Axillary lymph node stage of invasive } \\
\text { cancers, No (\%) }\end{array}$ & & & 0.26 \\
\hline Negative & $9(75.0)$ & $7(100)$ & \\
\hline Positive & $3(25.0)$ & $0(0)$ & \\
\hline Advanced cancer**, No (\%) & $4(30.8)$ & $2(25.0)$ & 1.00 \\
\hline
\end{tabular}

* Screen-film mammography: 1st and 2nd recall at screen-film mammography; full-field digital mammography: 1st recall at screen-film mammography, 2nd recall at full-field digital mammography; ** Invasive cancers with TNM stage T2a or higher, i.e. tumor size exceeding $20 \mathrm{~mm}$ and/or the presence of axillary metastasis $^{20}$

\section{Review of FFDM screens, with the availability of previously obtained hard copy SFM screens}

Review of the second recalls at FFDM, with the availability of the hard copy SFM screening examination in addition to the digitized SFM screens, would have resulted in 32 instead of 52 second recalls (a reduction of $39.5 \%$ of second recalls). These 32 recalls comprised 21 masses, 5 microcalcification abnormalities, 4 masses in combination with microcalfications and 2 architectural distortions. A significantly larger proportion of microcalfication abnormalities than masses would not have been recalled for a second time $(66.7 \%(10 / 15)$ versus $19.2 \%(5 / 26), P=0.006)$. None of the 20 women that would not have been recalled were diagnosed with breast cancer. The availability of the hard copy films would have resulted in a positive predictive value of second recall of $25.0 \%$ (8/32), which is comparable to the $35.1 \%(13 / 27)$ at SFM ( $P=0.36$, Figure 2.4$)$. 
Figure 2.4 Review of repeated recalls for the same mammographic abnormality at full-field digital mammography

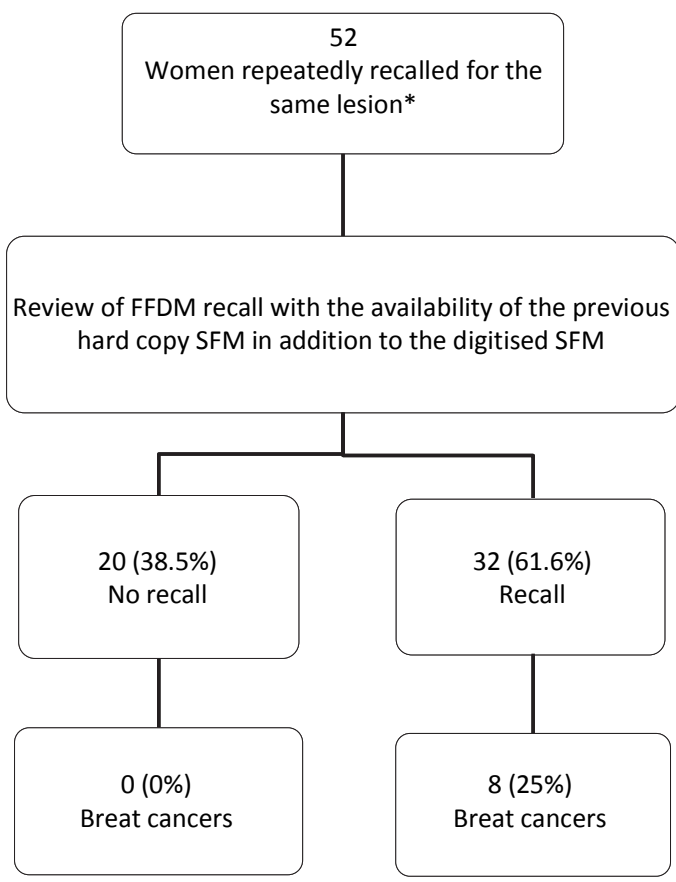

* 1st recall at SFM, 2nd recall at FFDM; SFM Screen-film mammography; FFDM Full-field digital mammography

\section{Discussion}

We found that the re-attendance rate was significantly lower for women who had experienced a repeated false positive recall, especially if both recalls comprised the same mammographic lesion. During the first screening round at FFDM, a significantly larger proportion of recalls included women who had been recalled twice for the same lesion and breast cancer was significantly less often diagnosed in these women than at SFM, with a concomitant lower positive predictive value of recall. However, the availability of the hard copy SFM screen, in addition to the digitized SFM screen, would have reduced the number of repeatedly recalled women at FFDM by almost $40 \%$.

To our knowledge, this is the first study that compares re-attendance rates of women recalled once or twice and that determines the effect of transition from SFM to FFDM on the proportion and screening outcome of women recalled twice for the same lesion. 
Whether or not receiving a single false positive mammogram influences attendance at subsequent screening mammography remains controversial. ${ }^{5-11}$ The current study shows that the re-attendance rates for women who had experienced a single false positive recall is significantly worse compared to patients with a negative screen at prior screening mammography (65.4\% versus 93.2\%). Re-attendance after a repeated false positive recall is even worse (52.1\%), especially if both recalls comprised the same mammographic lesion (44.3\%). This observation stresses the importance to minimize the number of false positives.

A recent study from our group showed that, at screen film mammography, $41.5 \%$ of the women recalled for a second time after a previous false positive screen, were recalled for the same lesion. ${ }^{22}$ In the Netherlands, the first introduction of FFDM was accompanied by higher cancer detection rates at the expense of higher recall rates and a decreased PPV. ${ }^{12-14}$ The current study shows that this decreased PPV not only involves women recalled only once, but also women recalled a second time for the same lesion.

Several studies have shown that FFDM results in an improved detection of cancers characterized by suspicious microcalcifications. ${ }^{12,23-27}$ In our overall population, a significantly larger proportion of FFDM recalls comprised suspicious microcalcifications at screening mammography. However, in patients recalled a second time for the same lesion, suspicious microcalcifications were less often the reason of recall at FFDM and, in contrast to SFM, none of these patients were diagnosed with breast cancer. This finding may be due to local contrast enhancement algorithms that enhance the mean level of suspicion of densities and focal asymmetries at FFDM. ${ }^{28}$

At FFDM, subsequent screens were compared with previous digitized SFM screens. Blinded review showed that the availability of the SFM hard copies would have greatly reduced the number of women recalled twice for the same mammographic abnormality. None of the women who would not have been recalled were ultimately diagnosed with breast cancer, resulting in a similar positive predictive value of recall for women recalled for the second time, either at SFM or FFDM. This observation underscores the importance of having prior screens available for comparison. Some mammographic abnormalities may come and go, so the availability of older images for comparison is crucial and can lower the recall rate. Failure to do so will increase the amount of false positive screens and eventually lower the re-attendance rate and the effectiveness of screening mammography.

We therefore suggest that, in case a women attends FFDM screening for the first time after previous SFM screening, the screening radiologist should have the opportunity to compare with the previous hard copy SFM examination if repeated recall is considered. Although FFDM has replaced SFM in most screening programs, this reading strategy may be of value for women who only have SFM screening examinations for comparison (e.g., screening programs currently converting to digital mammography and women who have skipped the digital screening rounds following their latest SFM screen). Due to limited data storage in the Netherlands, only two previous FFDM screening rounds will be available for comparison at successive FFDM 
screening mammography, whereas all prior screens were always available for comparison in the era of SFM screening mammography. The inability to compare with FFDM screening examinations dating back more than two screening rounds may thus put a strain on the attempts to reduce repeated false positive recalls.

Currently, screening technologists of our screening units inform the screening radiologists at each screen whether or not a woman has experienced a previous recall. This information may help the radiologist to decide if comparison with hard copy SFM or prior digital screens is desired.

Our study has certain limitations. Although all radiologists had more than five years of experience with working in a digital radiology environment, including digital mammography, none of them had experience with the use of FFDM in a screening setting at the start of FFDM screening. However, it is unlikely that our results have been influenced by a learning effect, as the recall rate, cancer detection rate and PPV of recall did not change during the digital screening period. ${ }^{12}$ Furthermore, the FFDM group was restricted to women who were digitally screened for the first time and we cannot predict the long-term impact on second recalls for the same lesion at successive digital screening rounds.

In summary, a repeated false positive recall for the same lesion significantly lowered the probability of screening re-attendance. The transition of SFM to FFDM significantly increased the proportion of recalls comprising women recalled twice for the same lesion and significantly lowered the positive predictive value of these recalls. The availability of the hard copy SFM screen, in addition to the digitized SFM screen, will likely reduce the proportion of repeatedly recalls of women at their first FFDM round. 


\section{References}

1 Dowling EC, Klabunde C, Patnick J, Ballard-Barbash R. Breast and cervical cancer screening programme implementation in 16 countries. J Med Screen 2010;17:139-146.

2 Duijm LE, Groenewoud JH, Fracheboud J et al. Utilization and cost of diagnostic imaging and biopsies following positive screening mammography in the southern breast cancer screening region of the Netherlands, 2000-2005. Eur Radiol 2008;18:2390-2397.

3 van der Steeg AF, Keyzer-Dekker CM, De Vries J, Roukema JA. Effect of abnormal screening mammogram on quality of life. Br J Surg 2011;98:537-542.

4 Brett J, Austoker J. Women who are recalled for further investigation for breast screening: psychological consequences 3 years after recall and factors affecting re-attendance. J Public Health Med 2001;23: 292-300.

5 Brewer NT, Salz T, Lillie SE. Systematic review: the long-term effects of false-positive mammograms. Ann Intern Med 2007;146:502-510.

6 Roman R, Sala M, De La Vega M et al. Effect of false-positives and women's characteristics on long-term adherence to breast cancer screening. Breast Cancer Res Treat 130:543-552.

7 Seigneurin A, Exbrayat C, Labarere J, Delafosse P, Poncet F, Colonna M. Association of diagnostic workup with subsequent attendance in a breast cancer screening program for false-positive cases. Breast Cancer Res Treat 2011;127:221-228.

8 Setz-Pels W, Duijm LE, Coebergh JW, Rutten M, Nederend J, Voogd AC. Re-attendance after falsepositive screening mammography: a population-based study in the Netherlands. Br J Cancer 2013; 109:2044-2050.

9 Alamo-Junquera D, Murta-Nascimento C, Macia F et al. Effect of false-positive results on reattendance at breast cancer screening programmes in Spain. Eur J Public Health 2012;22:404-408.

10 Andersen SB, Vejborg I, von Euler-Chelpin M. Participation behaviour following a false positive test in the Copenhagen mammography screening programme. Acta Oncol 2008;47:550-555.

11 Johns LE, Moss SM. False-positive results in the randomized controlled trial of mammographic screening from age 40 ("Age" trial). Cancer Epidemiol Biomarkers Prev 2010;19:2758-2764.

12 Nederend J, Duijm LE, Louwman MW, Groenewoud JH, Donkers-van Rossum AB, Voogd AC. Impact of transition from analog screening mammography to digital screening mammography on screening outcome in The Netherlands: a population-based study. Ann Oncol 2012;23:3098-3103.

13 Bluekens AM, Karssemeijer N, Beijerinck D et al. Consequences of digital mammography in populationbased breast cancer screening: initial changes and long-term impact on referral rates. Eur Radiol 2010;20:2067-2073.

14 van Luijt PA, Fracheboud J, Heijnsdijk EA, den Heeten GJ, de Koning HJ. Nation-wide data on screening performance during the transition to digital mammography: Observations in 6 million screens. Eur J Cancer 2013;49:3517-3525.

15 Fracheboud J, de Koning HJ, Boer R et al. Nationwide breast cancer screening programme fully implemented in The Netherlands. Breast 2001;10:6-11.

16 Duijm LE, Groenewoud JH, Hendriks JH, de Koning HJ. Independent double reading of screening mammograms in The Netherlands: effect of arbitration following reader disagreements. Radiology 2004;231:564-570.

17 Maes RM, Dronkers DJ, Hendriks JH, Thijssen MA, Nab HW. Do non-specific minimal signs in a biennial mammographic breast cancer screening programme need further diagnostic assessment? Br J Radiol 1997; 70:34-38.

18 Liberman L, Menell JH. Breast imaging reporting and data system (BI-RADS). Radiol Clin North Am 2002;40:409-+.

19 Sobin LH, Wittekind C. TNM Classification of Malignant Tumours. Wiley-Liss, New York 2002.

20 AJCC Cancer Staging Manual, Seventh Edition. Springer, New York 2010.

21 Duijm LE, Groenewoud JH, Jansen FH, Fracheboud J, van Beek M, de Koning HJ. Mammography screening in the Netherlands: delay in the diagnosis of breast cancer after breast cancer screening. $\mathrm{Br} J$ Cancer 2004;91:1795-1799. 
22 Setz-Pels W, Duijm LE, Louwman MW, Roumen RM, Jansen FH, Voogd AC. Characteristics and screening outcome of women referred twice at screening mammography. Eur Radiol 2012;22:2624-2632.

23 Feeley L, Kiernan D, Mooney $\mathrm{T}$ et al. Digital mammography in a screening programme and its implications for pathology: a comparative study. J Clin Pathol 2011;64:215-219.

24 Glynn CG, Farria DM, Monsees BS, Salcman JT, Wiele KN, Hildebolt CF. Effect of transition to digital mammography on clinical outcomes. Radiology 2011;260:664-670.

25 Del Turco MR, Mantellini $P$, Ciatto $S$ et al. Full-field digital versus screen-film mammography: comparative accuracy in concurrent screening cohorts. AJR Am J Roentgenol 2007;189:860-866.

26 Hambly NM, McNicholas MM, Phelan N, Hargaden GC, O'Doherty A, Flanagan FL. Comparison of digital mammography and screen-film mammography in breast cancer screening: a review in the Irish breast screening program. AJR Am J Roentgenol 2009;193:1010-1018.

27 Karssemeijer N, Bluekens AM, Beijerinck D et al. Breast cancer screening results 5 years after introduction of digital mammography in a population-based screening program. Radiology 2009;253:353-358.

28 Visser R, Veldkamp WJ, Beijerinck D et al. Increase in perceived case suspiciousness due to local contrast optimisation in digital screening mammography. Eur Radiol 2012;22:908-914. 


\section{Chapter 3}

Variations in screening outcome among pairs of screening radiologists at non-blinded double reading of screening mammograms: a population-based study

Klompenhouwer EG, Duijm LEM, Voogd AC, Heeten GJ, Nederend J, Jansen FH, Broeders MJM

Eur Radiol. 2014;24:1097-1104 


\section{Abstract}

Objectives

Substantial inter-observer variability in screening mammography interpretation has been reported at single reading. However, screening results of pairs of screening radiologists have not yet been published. We determined variations in screening performances among pairs of screening radiologists at non-blinded double reading.

\section{Methods}

We included pairs of screening radiologists with at least 7,500 screening examinations per pair, obtained between 1997 and 2011. During 2-year follow-up, breast imaging reports, surgical reports and pathology results were collected of all recalled women and interval cancers. Recall rate, cancer detection rate, positive predictive value and sensitivity were calculated for each pair.

\section{Results}

A total of 310,906 screening mammograms, read by 26 pairs of screening radiologists, were included for analysis. The recall rate ranged from $1.0 \%(95 \% \mathrm{Cl} 0.8 \%-1.2 \%)$ to $1.5 \%(95 \% \mathrm{Cl} 1.3 \%$ $1.8 \%)$, the cancer detection rate from $4.0(95 \% \mathrm{Cl} 2.8-5.2)$ to $6.3(95 \% \mathrm{Cl} 4.5-8.0)$ per 1,000 screens. The program sensitivity and positive predictive value of recall ranged from $55.1 \%(95 \% \mathrm{Cl}$ $45.1 \%-65.1 \%)$ to $81.5 \%(95 \% \mathrm{Cl} 73.4 \%-89.6 \%)$ and from $28.7 \%(95 \% \mathrm{Cl} 20.8 \%-36.6 \%)$ to $49.5 \%$ (95\% Cl 39.7\%-59.3\%), respectively.

\section{Conclusion}

We found significant variations in screening outcomes among pairs of screening radiologists at non-blinded double reading. This stresses the importance of monitoring screening results on a local scale. 


\section{Introduction}

Regional or nation-wide screening mammography programs have been introduced in many countries from the mid-1980s. ${ }^{1}$ Reading of screening mammograms can be performed in several ways. Many programs use single reading by a radiologist, with or without computer-aided detection (CAD). A United Kingdom study showed that single reading with CAD could be an alternative to double reading, improving the rate of cancer detection from screening mammograms read by a single reader. ${ }^{2}$ On the other hand, Fenton et al. concluded that the use of CAD in the United States (US) was associated with decreased specificity, but not with improvement in the detection rate or prognostic characteristics of invasive breast cancer. ${ }^{3,4}$ Non-blinded, or independent, double reading significantly increases the cancer detection rate when compared to single reading and is considered to be the standard of reference for the assessment of screening mammograms. ${ }^{5-7}$ It is a question of debate whether or not double reading also increases recall rates. ${ }^{6,8} \mathrm{~A}$ systematic review showed that arbitration of discrepant findings between two radiologists at double reading helps to decrease recall rates, whereas adding CAD to single reading did not improve the cancer detection rate and increased the recall rate. ${ }^{9}$ Reading of screening mammograms by mammography technologists, in addition to radiologist single reading or radiologist double reading, also increases screening sensitivity, at the cost of a small decrease in specificity. ${ }^{10,11}$ Additional assessment by technologists or CAD may be considered when radiologist double reading is not feasible due to a shortage of trained and experienced radiologists. $^{12,13}$

In the Netherlands, a nation-wide program provides biennial screening mammography for women since 1995. All mammograms are consistently double-read, routinely in a non-blinded fashion (i.e., the second reader is not blinded to the first reader's opinion). In case of a discrepant reading, the two radiologists may discuss the case together to reach consensus about recall, a third reader may be added for arbitration, or the woman may routinely be recalled without consensus reading or arbitration. $^{14}$

Substantial inter-observer variability in screening mammography interpretation among screening radiologists has well been documented. ${ }^{15,16}$ However, to our knowledge, population based studies on the screening results of pairs of screening radiologists have not yet been published. Therefore, the aim of the current study was to determine variations in screening performances among unique pairs of screening radiologists at non-blinded double reading of screening mammograms, including: recall rate, cancer detection rate, sensitivity and positive predictive value of recall. 


\section{Materials and methods}

\section{Study population}

The study population included a consecutive series of 389427 screens that had been non-blinded double read by certified screening radiologists. The screens were obtained at two screening units in a southern breast cancer screening region of the Netherlands (BOZ, Bevolkings Onderzoek Zuid) between January 1, 1997 and January 1, 2011. For the purpose of the current study we included pairs with at least 7,500 screening examinations per pair in order to be able to perform statistical analysis on the outcomes of screening pairs.

Women participating in the nationwide Dutch screening program are asked to give written informed consent for the use of their data for scientific purposes. Four women screened at our two units did not give informed consent and they were excluded from the analysis. The study obtained a waiver from the Dutch Central Committee on Research Involving Human Subjects (CCMO), which is an organization supervising the ethical review of screening trials performed within the Netherlands.

\section{Screening procedure and recall}

Details of the nationwide breast cancer screening program, offering biennial screening mammography, have been described previously. ${ }^{14,15}$ In brief, this program is offered since 1995 for women aged 50-69 years and since 1998 for women aged 50-75 years. Screen-film mammograms were obtained with commercially available units (Performa, Oldelft, Tuusula, Finland). Dedicated mammography screens were utilized (Mamoray MR-R, Agfa, Schroenhausen, Germany). Both film (Mamoray HDR; Agfa, Mortsel, Belgium), as well as extended-cycle processing were used. In June 2009, screen-film mammography was replaced by full-field digital mammography. All digital mammograms were acquired with a Lorad Selenia FFDM system (Hologic Inc, Danbury, $\mathrm{CT}$ ), with a $70 \mu \mathrm{m}$ pixel size and a $232 \times 286 \mathrm{~mm}$ field of view. The mammographic examinations were obtained by specialized screening mammography technologists and double read by a team of 8 certified screening radiologists. Prior screening mammograms were always available for comparison at the time of a subsequent screening round and each radiologist evaluated at least 3,000 screening mammograms yearly. Women with normal or benign mammographic findings, or with a non-specific minimal sign, ${ }^{16}$ were not recalled. If screening mammography showed a suspicious or malignant lesion, the woman was recalled to a surgical oncologist or breast clinic for further analysis of the mammographic abnormality.

\section{Follow-up procedure}

The follow-up period for all screened women included the time through the next screening round, with a screening interval of approximately 2 years. In addition to the 
data collected by the screening organization, one of the radiologists (L.E.M.D) yearly visits the regional hospitals to obtain a two year follow-up of diagnostic tests and final screening outcome of recalled women. Breast cancers were divided into ductal carcinoma in situ (DCIS) and invasive cancers according to the TNM (tumor-nodemetastases) classification; ${ }^{17}$ lobular carcinoma in situ was considered to be a benign lesion. Procedures for the detection of interval cancers have been described previously. ${ }^{18}$ An interval cancer is a breast cancer that is diagnosed after a negative screening mammography (that is, screening without a recommendation for recall) and before the next biennial screening examination. These can be cancers which were missed by screening, cancers which became screen detectable after the screen and then became symptomatic, and cancers not detectable by mammography. ${ }^{19}$ To determine whether the interval cancer could be attributed to the radiologic assessment, two screening radiologists (L.E.M.D., F.H.J., with 13 and 15 years of screening experience, respectively) reviewed the screening mammogram and clinical mammogram of all women with a diagnosis of interval breast cancer. They determined whether the interval cancers had been missed or had been mammographically occult at the time of the latest screen. Each reviewer classified the lesions using the Breast Imaging Reporting and Data System, BI-RADS. ${ }^{20}$ For BI-RADS 3 lesions (i.e., nonpalpable, probably benign lesions), the criteria published by Sickles ${ }^{21}$ and Varas et al. ${ }^{22}$ were used. At review, the radiologists were initially blinded to the each other's opinion and discrepant assessments were followed by consensus reading.

\section{Participating radiologists and quality assurance}

Of the 8 participating radiologists, 5 started with screening mammography in 1995, whereas the remaining 3 radiologists entered the screening program in respectively 1997, 1998 and 1999. Four of the 8 participating radiologists are also involved in diagnostic breast imaging (radiologist $c, d, f$ and $h$ ) and two of them are specialized in breast imaging (radiologist $\mathrm{c}$ and $\mathrm{f}$ ). These two dedicated breast radiologists coordinate the screening mammography program and breast radiology fellowship.

Dutch screening radiologists receive certification in screening mammography after an 8 days film reading training at the National Expert and Training Centre for Breast cancer screening (NETCB).

Since 1997, all screening radiologists of our screening group attend plenary sessions every 3-4 months for review of interval cancers. Since 2009, all radiologists receive feedback on their individual screening performance bi-annually, including data on recall rate, cancer detection rate, sensitivity and positive predictive value of recall.

All specialized mammography technologists in the Netherlands also receive extensive training at the NETCB prior to their employment as screening technologists and they attend mammography symposia and conferences every 1 to 2 years. The technologists are encouraged to make a note of suspicious mammographic abnormalities. At regular quality assurance sessions, two screening radiologists (preferably those who have performed the original assessment) review those cases that 
only the technologists have considered suspicious. The woman is still recalled if at least one of the reviewing radiologists considers recall necessary.

\section{Statistical analysis}

The main outcome measures were recall rate, cancer detection rate (CDR), sensitivity and positive predictive value (PPV) of recall for each unique pair of screening radiologists. Sensitivity per screening pair was calculated in two ways: 1) based on all interval cancers (program sensitivity) and 2) based on missed interval cancers only (mammographic sensitivity). For the purpose of the current study, cancers that had been detected following review at the quality assurance sessions (and thus initially missed by the screening radiologists) were considered missed cancers. The cancer incidence was calculated as the total of screen detected cancers and interval cancers, divided by the total number of screens. A double-sided t-test was used to test differences between pairs, 95\% confidence intervals $(\mathrm{Cl})$ were calculated and Pearson's correlation coefficient was calculated for correlations between screening results of pairs. A Chi-square test was used to test differences in mammographic and tumor characteristics between screen detected cancers and missed cancers.

All data were entered into a computerized spreadsheet (Excel; Microsoft, Redmond, WA, USA). Statistics were performed using Statistical Package for Social Science 17.0 (SPSS Inc. Chicago, IL). A $P$ value of less than 0.05 was considered to indicate statistical significance.

\section{Results}

A total of 27 pairs (8 radiologists) had read at least 7500 screens per pair. One pair had a significant lower cancer incidence $(5.5 \%, 95 \% \mathrm{Cl} 3.9 \%$ - $6.8 \%$ ) compared to the other pairs and was therefore excluded from analysis. The remaining 26 pairs ( 8 radiologist) had assessed a total of 310906 screening examinations (Figure 3.1).

The number of screens per pair ranged from 7791 to 20920 screens. The mean cancer incidence of the 26 pairs was $7.2 \%$ o $(95 \% \mathrm{Cl} 6.9 \%$ o-7.5\%o), and ranged from $6.0 \%$ $(95 \% \mathrm{Cl} 4.7 \%$ - $7.3 \%$ ) to $8.9 \%$ o (95\% Cl 6.9\%o-11.0\%o) among pairs (Figure 3.2). The mean proportion of initial screens among the pairs was $12.8 \%(95 \% \mathrm{Cl} 12.7-12.9)$, and ranged from 12.5 (95\% Cl 11.9\%-13.1\%) and 13.0 (95\% Cl 12.5-13.8).

The recall rate among pairs ranged from $1.0 \%(95 \% \mathrm{Cl} 0.8 \%-1.2 \%)$ to $1.5 \%(95 \% \mathrm{Cl}$ $1.3 \%-1.8 \%)$, with an overall mean recall rate of $1.3 \%(95 \% \mathrm{Cl} 1.2 \%-1.3 \%)$ (Figure 3.2$)$. 


\section{Figure 3.1 Study population}

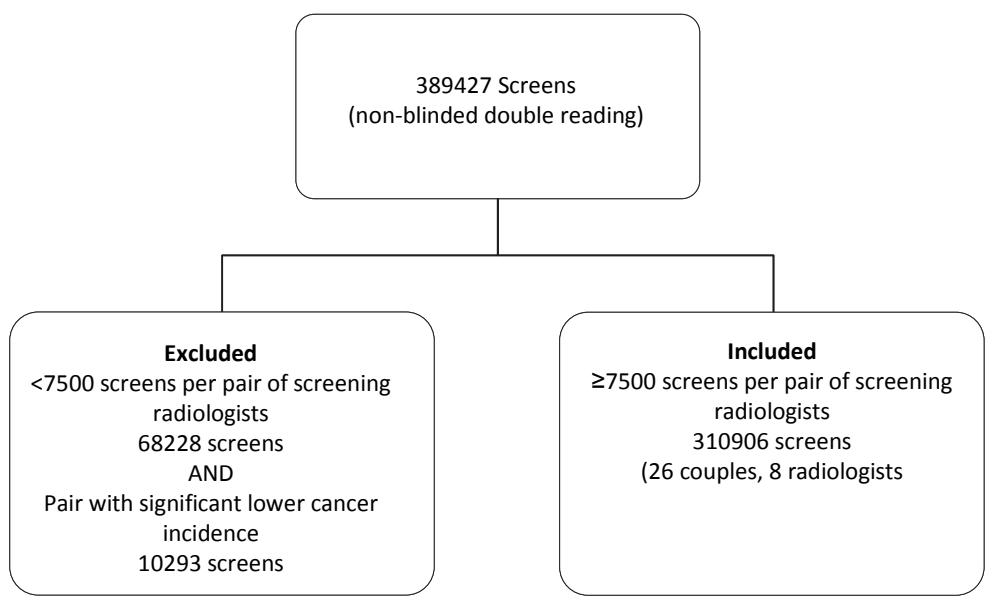

Figure 3.2 Number of screen, breast cancer incidence and recall rate for 26 pairs of screening radiologists (8 radiologists $\mathrm{a}-\mathrm{h}$ )

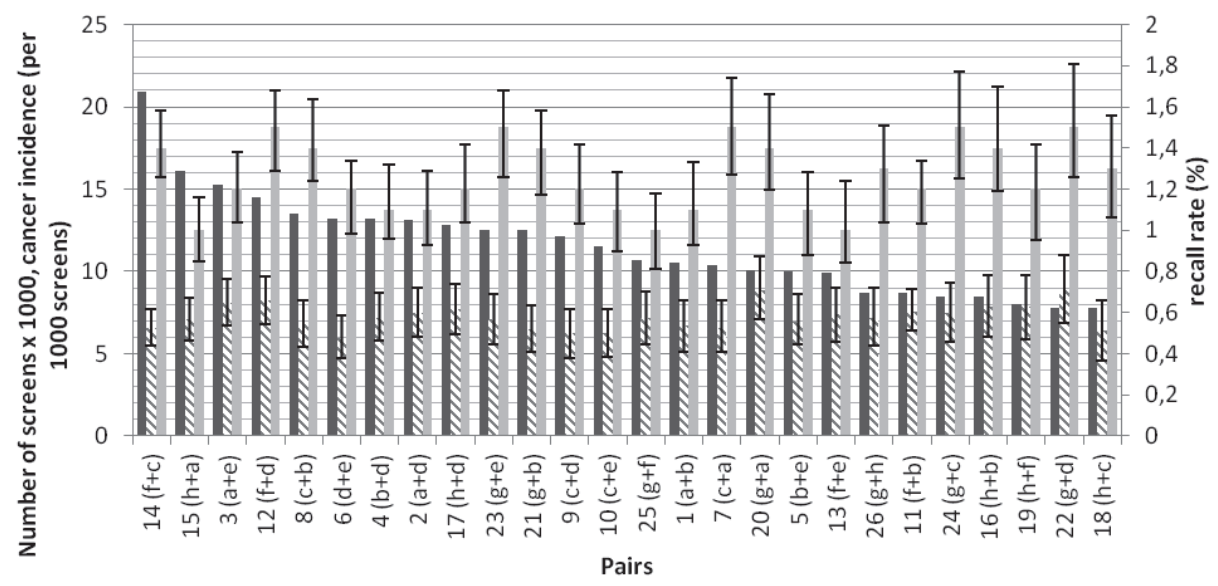

- Screens $\quad$ NCancer incidence $\quad$ Recall rate

I-----l The vertical lines show the $95 \% \mathrm{Cl}$

The mean overall CDR was $4.9(95 \% \mathrm{Cl} 4.7-5.2)$ and ranged from $4.0(95 \% \mathrm{Cl} 2.8-5.2)$ to $6.3(95 \% \mathrm{Cl} 4.5-8.0)$ per 1,000 screens among pairs of screening radiologists (Figure 3.3).

There were a total of 1553 women with a screen detected cancer, comprising 260 screen detected DCIS $(16.7 \%, 95 \% \mathrm{Cl} 14.9 \%-18.6 \%), 1010 \mathrm{~T} 1 \mathrm{a}-\mathrm{c}$ tumors $(65 \%, 95 \% \mathrm{Cl}$ $62.7 \%-67.4 \%)$ and $283 \mathrm{~T} 2+$ tumors $(18.2 \%, 95 \% \mathrm{Cl} 16.3 \%-20.1 \%)$. The amount of screen 
detected DCIS, T1a-c tumors en T2+ tumors did not differ significantly among pairs with a range of $10.5 \%(95 \% \mathrm{Cl} 3.0 \%-20.8 \%)$ to $23.2 \%(95 \% \mathrm{Cl} 13.0-33.4 \%), 49 \%(95 \% \mathrm{Cl} 34.4 \%$ $63.5 \%)$ to $71.1 \%(95 \% \mathrm{Cl} 62.0 \%-80.3 \%)$, and $10 \%(95 \% \mathrm{Cl} 0.3 \%-19.7 \%)$ to $28.5 \%(95 \% \mathrm{Cl}$ $15.5 \%-41.7 \%)$, respectively.

Figure 3.3 Cancer detection rate and positive predictive value of recall among 26 pairs of screening radiologists ( 8 radiologists $\mathrm{a}-\mathrm{h}$ )
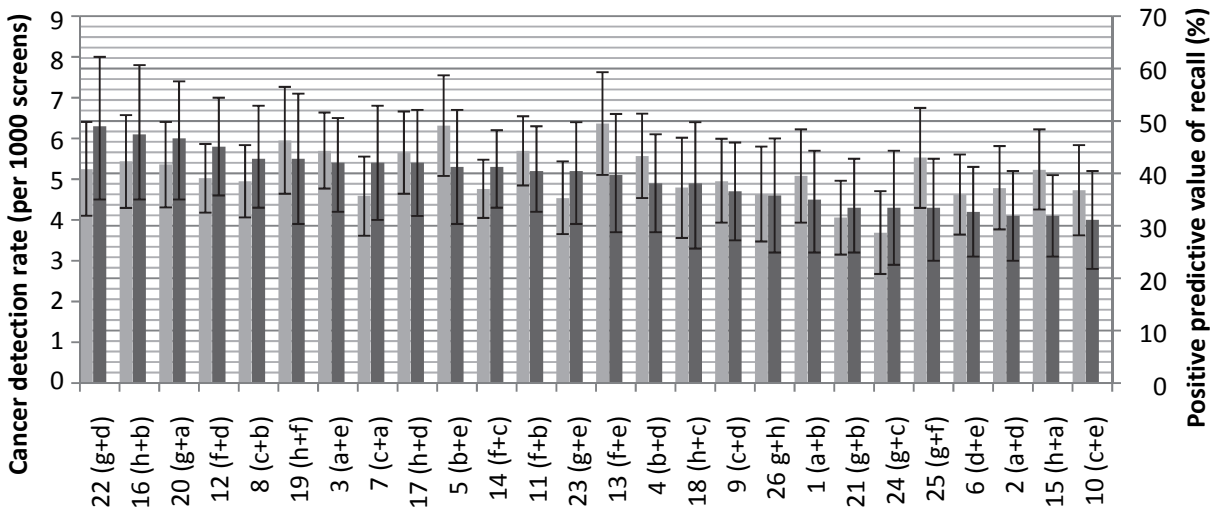

Pairs

Cancer detection rate

- Positive predictive value of recall

I-----l The vertical lines show the $95 \% \mathrm{Cl}$

The PPV of recall (Figure 3.3) ranged from $28.7 \%$ (95\% Cl 20.8\%-36.6\%, screening pair 23$)$ to $49.5 \%(95 \% \mathrm{Cl} 39.7 \%-59.3 \%$, screening pair 13$)$ with a mean of $39.5 \%(95 \% \mathrm{Cl}$ $38.0 \%-41.0 \%)$. The CDR was significantly related to the recall rate (Pearson's correlation coefficient $r=0.52, P=0.007)$.

There were a total 628 interval cancer cases, 155 (24.7\%) of these cancers were considered to be missed cancers at review. There were another 65 cancers that had been detected through the quality assurance sessions and considered as missed interval cancer for the purpose of this study. The mammographic and tumor characteristics differed significantly between screen detected cancers and missed cancers (Table 3.1). Missed cancers presented more often as architectural distortion or asymmetry, whereas screen detected cancers presented more often as suspicious microcalcifications. Lobular cancers and mixed ductal/lobular cancers were more often missed, whereas DCIS was more often detected at breast cancer screening. Patients with a missed cancer showed a significantly higher breast density at screening mammography 
Table 3.1 Mammographic and tumor characteristics of screen detected cancers and missed cancers

\begin{tabular}{|c|c|c|c|}
\hline & $\begin{array}{l}\text { Screen detected } \\
\text { cancers }(n=1553)\end{array}$ & $\begin{array}{l}\text { Missed cancers * } \\
\qquad(n=220)\end{array}$ & $P$-value \\
\hline Mammographic abnormality, n (\%) & & & $<0.001$ \\
\hline Mass & $1040(67.0)$ & $147(66.8)$ & \\
\hline Microcalcifications & $286(18.4)$ & $29(13.2)$ & \\
\hline Mass with microcalcifications & $175(11.3)$ & $19(8.6)$ & \\
\hline Asymmetry & $12(0.8)$ & $18(8.2)$ & \\
\hline Architectural distortion & $40(2.6)$ & $7(3.2)$ & \\
\hline Breast density at screening mammography & & & $<0.001$ \\
\hline $0 \%-25 \%$ & $576(37.1)$ & $49(22.3)$ & \\
\hline $25-50 \%$ & $490(31.6)$ & $86(39.1)$ & \\
\hline $50-75 \%$ & $351(22.6)$ & $61(27.7)$ & \\
\hline $75-100 \%$ & $136(8.8)$ & $27(12.3)$ & \\
\hline Breast cancers, histologic type, n (\%) & & & 0.02 \\
\hline Ductal carcinoma in situ & $260(16.7)$ & $20(9.1)$ & \\
\hline Invasive ductal cancer & $989(63.7)$ & $141(64.1)$ & \\
\hline Invasive lobular cancer & $152(9.8)$ & 35 (15.9) & \\
\hline Invasive mixed ductal/lobular cancer & $64(4.1)$ & $14(6.4)$ & \\
\hline Invasive, other & $82(5.3)$ & $8(3.6)$ & \\
\hline Unknown & $6(0.4)$ & $2(0.9)$ & \\
\hline
\end{tabular}

Both the program and mammographic sensitivity differed significantly among pairs. The mean program sensitivity was $68.9(95 \% \mathrm{Cl} 67.2 \%-71.1 \%)$ and ranged from $55.1 \%$ $(95 \% \mathrm{Cl} 45.1 \%-65.1 \%)$ to $81.5 \%(95 \% \mathrm{Cl} 73.4 \%-89.6 \%)$. The mean mammographic sensitivity was $87.6 \%(95 \% \mathrm{Cl} 86.1 \%-89.1 \%)$ and ranged from $72.0 \%(95 \% \mathrm{Cl} 61.6 \%$ $82.4 \%$ ) to $96.6 \%$ (95\%Cl 91.7\%-101.4\%) (Figure 3.4).

Screening pair 2 showed a significantly lower program and mammographic sensitivity and pair 15 showed a significant worse program sensitivity compared to the other pairs. Screening pair 7 showed a significant better mammographic and program sensitivity and pair 8 and 14 showed a significant better program sensitivity compared to the other pairs.

Individual pairs showed no correlation between number of screens and sensitivity (program sensitivity, Pearson's correlation coefficient $r=0.04, P=0.84$; mammographic sensitivity, Pearson's correlation coefficient $r=-0.30, P=0.13$ ), number of screens and PPV (Pearson's correlation coefficient $r=0, P=0.98$ ), or sensitivity and PPV (program sensitivity, Pearson's correlation coefficient $r=0.13, P=0.53$; mammographic sensitivity, Pearson's correlation coefficient $r=0.05, P=0.80$ ) 
Figure 3.4 Screening sensitivity of 26 pairs of screening radiologists (8 radiologists a-h)

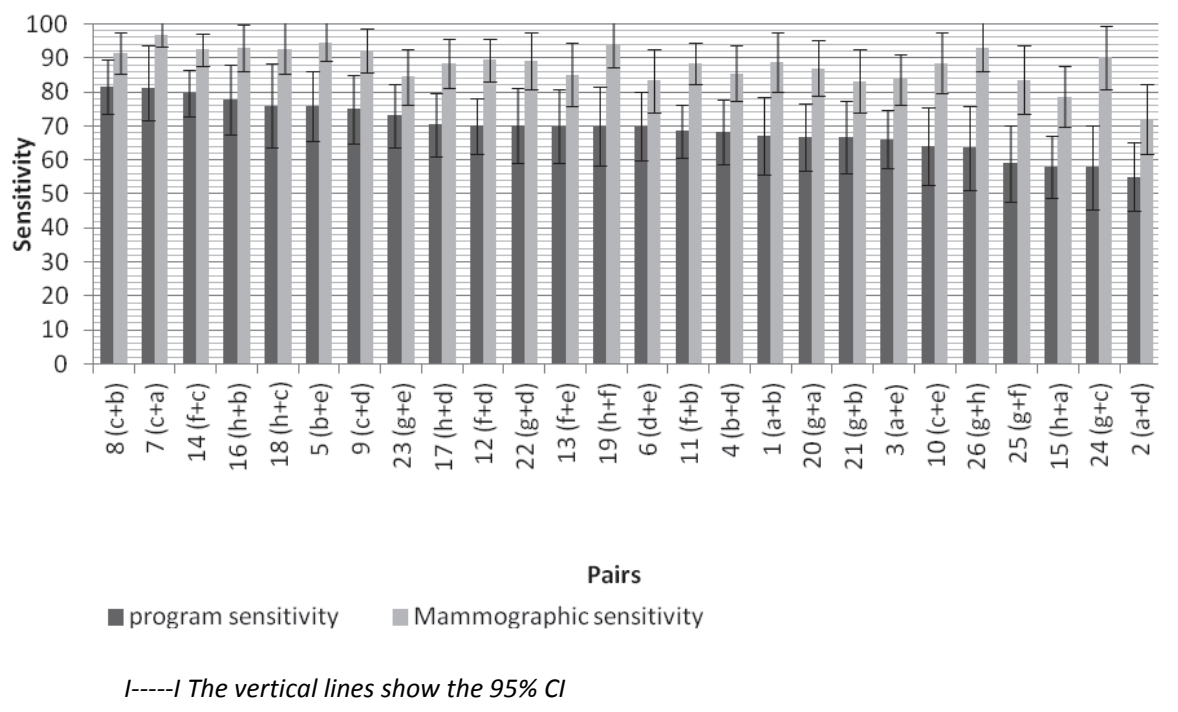

\section{Discussion}

An important indicator in the evaluation of screening programs is the program sensitivity. The sensitivity is influenced by a number of parameters including screening interval, age of the target population, mean speed of growth of the mix of tumors, the technical level of the screening test and the ability of radiologists to detect early signs of cancer growth. This paper focuses on the variability in the specific set up of nonblinded double reading.

In 2009 we reported a significant inter-observer variation in the individual screening performances among radiologists. ${ }^{23-25}$ We expected that the addition of a second reader would solve these variations in screening outcome, however our results proved otherwise. Variations in screening performance significantly varied among 26 pairs of screening radiologists with respect to recall rate, program and mammographic sensitivity and PPV of recall. The CDR was significantly related to the recall rate and individual pairs showed no correlation between number of screens and sensitivity, number of screens and PPV, or sensitivity and PPV.

The data of the current study are based on a group of 8 screening radiologists who assessed screening mammograms at two screening units in a southern breast cancer screening region of the Netherlands. All 8 radiologists work together at a large teaching hospital and each of them participates equally in quality assurance programs. Several studies show that higher annual volumes improve screening results ${ }^{26,27}$ and each radiologist in our study performed a high annual screening volume of at least 3000 screening mammograms. These working conditions suggest that their contributions to 
the skill mix should level off, but nevertheless we found significant inter-pair variability in screening outcome with respect to recall rate, sensitivity and PPV of recall.

Literature shows a positive correlation between recall rate and $C D R$ for the individual radiologist. ${ }^{24,28}$ Our results showed that higher recall rates were significantly correlated with increased detection rates among pairs as well. A positive relationship has been reported between CDR and radiologists performing more diagnostic workup $^{26}$ and between CDR and radiologists with fellowship training in breast imaging. ${ }^{29}$ These results correspond to the belief that regular feedback improves performance. A radiologist who interprets screening examinations without the opportunity to know the outcome of recalled women may be hampered to improve his recall behavior. Radiologists who also perform diagnostic work-up of recalled women, including radiologists with fellowship training in breast imaging, build up their experience by assessing a case from screening through work-up till final diagnosis. Although our numbers are too small to calculate these correlations among our screening pairs, we found significantly better sensitivities in three screening pairs, and each of them consisted of at least one dedicated breast radiologist. We found significantly worse sensitivities in two screening pairs. These pairs consisted of one radiologist involved in diagnostic breast imaging and one radiologist not involved in diagnostic breast imaging, but no dedicated breast radiologist. It may be desirable to create specific pairs of radiologists in which radiologists specialized in breast imaging are combined with radiologists who are not. However, this may not be feasible in daily practice due to the working schedules of screening radiologists.

Carney et al. ${ }^{30}$ described minimally acceptable interpretive performance criteria for screening mammography for individual screening radiologists. These criteria included sensitivity more than $75 \%$, a $88 \%-95 \%$ specificity, a $5 \%-12 \%$ recall rate, a PPV of recall less between $3 \%$ and $8 \%$ and a cancer detection rate of at least 2.5 per 1000 interpretations. Doyle et al. ${ }^{31}$ argued that these criteria were of limited value and that it is not clear if the indicators are relevant for all patient populations. Carney's criteria cannot be extrapolated to the Dutch screening setting as recall rates in the Netherlands are much lower than the ones observed in the US (1-2\% versus 5-12\% respectively $\left.{ }^{32,33}\right)$, with consequently a higher PPV of recall and relative lower sensitivities. Further, we related screening outcome parameters to the total breast cancer incidence after two year follow-up instead of after one year follow-up, as this will provide full information about interval cancer rate in biennial screening. Finally, the criteria were developed for individual screening radiologists in a setting of single reading and not for screening pairs. Nevertheless, it seems a good suggestion of Carney et al. ${ }^{30}$ that pairs with suboptimal screening results can be identified and offered additional training. In our study population, missed interval cancers presented more often as architectural distortion or asymmetry, and comprised a significantly higher proportion of invasive lobular and mixed ductal/lobular cancers. These findings suggest that more attention should be paid to asymmetries and architectural distortions as possible signs of malignancy at screening mammography. 
The breast density of patients with a missed cancer was significantly higher compared to patients with a screen detected cancer. Studies from the US have shown that digital mammography improves the cancer detection in patients with dense breasts when compared to screen-film mammography. ${ }^{34,35}$ We hope that the conversion from screen-film to full-field digital mammography at our screening region, which took place in 2009, will also lead to a better cancer detection in women with dense breasts.

The Dutch National Expert and Training Centre for Breast cancer screening advises nowadays a target recall rate of $2.5-3 \%$ for initial screens and $1.3-2 \%$ for subsequent screens. Our $1.3 \%$ mean recall rate is relatively low, but the study population was limited to screens performed from 1997 to 2011. At the beginning of our inclusion period, the recall rate in the Netherlands was less than $1 \%$ for subsequent screens[36]. Since the optimization study of Otten et al. in 2005, which showed an obvious positive effect of increasing recall rates up to $4 \%$ on the cancer detection rate, ${ }^{37}$ and the implementation of digital screening mammography in 2009, our mean recall rate has increased to $3 \% .^{38}$

Our study has certain limitations. First, three of the participating radiologists started breast cancer screening during the inclusion period. These radiologists will have experienced a learning curve for the assessment of screening mammograms. ${ }^{39}$ However, the influence of this learning period on our results is probably limited as the inclusion period was 14 years. During our inclusion period, there have been some changes in our screening protocol, for example screen-film mammography was replaced by full-field digital mammography in 2009 and quality assurance was improved. However, all pairs of screening radiologists were exposed to these changes and no new pairs entered our screening program after these changes. So it is not to be expected that these changes will have significantly influenced our results.

Unfortunately, we were not able to identify reasons for the lower cancer incidence of one screening couple. Although we have details on age, breast density, hormone replacement therapy, previous breast surgery (benign or malignant) and family history of breast cancer of women with screen detected cancer or interval cancer, these data are not available for the whole study population. Finally, the second reader was not blinded to the first reader's opinion in the era of screen-film screening mammography. With the introduction of digital screening it became possible to perform blinded double reading instead of non-blinded double reading. Research data on blinded versus nonblinded double reading of screening mammograms on screening outcome will soon become available.

In conclusion, we found significant variations in screening outcomes among unique pairs of screening radiologists. In order to detect suboptimal results of screening pairs it is important to monitor screening results on a local scale and to continuously update interval cancers at screening mammography programs. 


\section{References}

1 Shapiro S, Coleman EA, Broeders M et al. Breast cancer screening programmes in 22 countries: current policies, administration and guidelines. International Breast Cancer Screening Network (IBSN) and the European Network of Pilot Projects for Breast Cancer Screening. Int J Epidemiol 1998;27:735-742

2 Gilbert FJ, Astley SM, Gillan MG et al. Single reading with computer-aided detection for screening mammography. N Engl J Med 2008;359:1675-1684

3 Fenton JJ, Taplin SH, Carney PA et al. Influence of computer-aided detection on performance of screening mammography. N Engl J Med 2007;356:1399-1409

4 Fenton JJ, Abraham L, Taplin SH et al. Effectiveness of computer-aided detection in community mammography practice. J Natl Cancer Inst 2011;103:1152-1161

5 Brown J, Bryan S, Warren R. Mammography screening: an incremental cost effectiveness analysis of double versus single reading of mammograms. BMJ 1996;312:809-812

6 Harvey SC, Geller B, Oppenheimer RG, Pinet M, Riddell L, Garra B. Increase in cancer detection and recall rates with independent double interpretation of screening mammography. AJR Am J Roentgenol 2003;180:1461-1467

7 Duijm LE, Groenewoud JH, de Koning HJ et al. Delayed diagnosis of breast cancer in women recalled for suspicious screening mammography. Eur J Cancer 2009;45:774-781

8 Thurfjell EL, Lernevall KA, Taube AA. Benefit of independent double reading in a population-based mammography screening program. Radiology 1994;191:241-244

9 Taylor P, Potts HW. Computer aids and human second reading as interventions in screening mammography: two systematic reviews to compare effects on cancer detection and recall rate. Eur J Cancer 2008;44:798-807

10 Duijm LE, Groenewoud JH, Roumen RM, de Koning HJ, Plaisier ML, Fracheboud J. A decade of breast cancer screening in The Netherlands: trends in the preoperative diagnosis of breast cancer. Breast Cancer Res Treat 2007;106:113-119

11 Tonita JM, Hillis JP, Lim CH. Medical radiologic technologist review: effects on a population-based breast cancer screening program. Radiology 1999;211:529-533

12 Duijm LE, Groenewoud JH, Fracheboud J, van Ineveld BM, Roumen RM, de Koning HJ. Introduction of additional double reading of mammograms by radiographers: effects on a biennial screening programme outcome. Eur J Cancer 2008;44:1223-1228

13 Karssemeijer N, Bluekens AM, Beijerinck D et al. Breast cancer screening results 5 years after introduction of digital mammography in a population-based screening program. Radiology 2009;253:353-358

14 Duijm LE, Groenewoud JH, Hendriks JH, de Koning HJ. Independent double reading of screening mammograms in The Netherlands: effect of arbitration following reader disagreements. Radiology 2004;231:564-570

15 Fracheboud J, de Koning HJ, Boer R et al. Nationwide breast cancer screening programme fully implemented in The Netherlands. Breast 2001;10:6-11

16 Maes RM, Dronkers DJ, Hendriks JH, Thijssen MA, Nab HW. Do non-specific minimal signs in a biennial mammographic breast cancer screening programme need further diagnostic assessment? $\mathrm{Br} \mathrm{J}$ Radiol 1997;70:34-38

17 Sobin LH, Wittekind C. TNM Classification of Malignant Tumours. Wiley-Liss, New York. 2002.

18 Duijm LE, Groenewoud JH, Jansen FH, Fracheboud J, van Beek M, de Koning HJ. Mammography screening in the Netherlands: delay in the diagnosis of breast cancer after breast cancer screening. $\mathrm{Br} \mathrm{J}$ Cancer 2004;1:1795-1799

19 Warren R, Duffy S. Interval cancers as an indicator of performance in breast screening. Breast Cancer 2000;7:9-18

20 Liberman L, Menell JH. Breast imaging reporting and data system (BI-RADS). Radiol Clin North Am 2002;40:409-+

21 Sickles EA. Periodic mammographic follow-up of probably benign lesions: results in 3,184 consecutive cases. Radiology 179:463-468 
22 Varas X, Leborgne JH, Leborgne F, Mezzera J, Jaumandreu S, Leborgne F. Revisiting the mammographic follow-up of BI-RADS category 3 lesions. AJR Am J Roentgenol 2002;179:691-695

23 Beam CA, Conant EF, Sickles EA. Association of volume and volume-independent factors with accuracy in screening mammogram interpretation. J Natl Cancer Inst 2003;95:282-290

24 Duijm LE, Louwman MW, Groenewoud JH, van de Poll-Franse LV, Fracheboud J, Coebergh JW. Interobserver variability in mammography screening and effect of type and number of readers on screening outcome. Br J Cancer 2009;100:901-907

25 Elmore JG, Miglioretti DL, Reisch LM et al. Screening mammograms by community radiologists: variability in false-positive rates. J Natl Cancer Inst 2002;94:1373-1380

26 Buist DS, Anderson ML, Haneuse SJ et al. Influence of annual interpretive volume on screening mammography performance in the United States. Radiology 2011;259:72-84

27 Esserman L, Cowley $\mathrm{H}$, Eberle $\mathrm{C}$ et al. Improving the accuracy of mammography: volume and outcome relationships. J Natl Cancer Inst 2002;94:369-375

28 Gur D, Sumkin JH, Hardesty LA et al. Recall and detection rates in screening mammography. Cancer 2004;100:1590-1594

29 Elmore JG, Jackson SL, Abraham L et al. Variability in interpretive performance at screening mammography and radiologists' characteristics associated with accuracy. Radiology 2009;253:641-651

30 Carney PA, Sickles EA, Monsees BS et al. Identifying minimally acceptable interpretive performance criteria for screening mammography. Radiology 2010;255:354-361

31 Doyle GP, Onysko J, Pogany L et al. Limitations of minimally acceptable interpretive performance criteria for screening mammography. Radiology 22011;58:960-961

32 Screening NETfBC. National evaluation of breast cancer in the Netherlands 1990-2007. 12th evaluation report. Erasmus Medical Center, Rotterdam. 2009.

33 Smith-Bindman R, Chu PW, Miglioretti DL et al. Comparison of screening mammography in the United States and the United kingdom. JAMA 2003;290:2129-2137

34 Kerlikowske K, Hubbard RA, Miglioretti DL et al. Comparative effectiveness of digital versus film-screen mammography in community practice in the United States: a cohort study. Ann Intern Med 2011;155:493-502

35 Pisano ED, Gatsonis C, Hendrick E et al. Diagnostic performance of digital versus film mammography for breast-cancer screening. N Engl J Med 2005;353:1773-1783

36 Fracheboud J, de Koning HJ, Beemsterboer PM et al. Nation-wide breast cancer screening in The Netherlands: results of initial and subsequent screening 1990-1995. National Evaluation Team for Breast Cancer Screening. Int J Cancer 1998;75:694-698

37 Otten JD, Karssemeijer N, Hendriks JH et al. Effect of recall rate on earlier screen detection of breast cancers based on the Dutch performance indicators. J Natl Cancer Inst 2005;97:748-754

38 Nederend J, Duijm LE, Louwman MW et al. Impact of the transition from screen-film to digital screening mammography on interval cancer characteristics and treatment - a population based study from the Netherlands. Eur J Cancer 2014;50:31-39

39 Miglioretti DL, Gard CC, Carney PA et al. When Radiologists Perform Best: The Learning Curve in Screening Mammogram Interpretation. Radiology 2009;253:632-640 


\section{Chapter 4}

Blinded versus non-blinded double reading 


\section{Chapter 4.1}

Blinded double reading yields a higher program sensitivity than non-blinded double reading at digital screening mammography: a prospective populationbased study in the south of the Netherlands

Klompenhouwer EG, Voogd AC, den Heeten GJ, Strobbe LJA, de Haan AF, Wauters CA, Broeders MJM, Duijm LEM

Eur J Cancer. 2015;51(3):391-9 


\section{Abstract}

\section{Purpose}

To prospectively determine the screening mammography outcome at blinded and non-blinded double reading in a biennial population-based screening program in the south of the Netherlands.

\section{Methods}

We included a consecutive series of 87,487 digital screening mammograms, obtained between July 2009 and July 2011. Screening mammograms were double read in either a blinded $\left(2^{\text {nd }}\right.$ reader was not informed about the $1^{\text {st }}$ reader's decision) or non-blinded fashion $\left(2^{\text {nd }}\right.$ reader was informed about the $1^{\text {st }}$ reader's decision). This reading strategy was alternated on a monthly basis. Women with discrepant readings between the two radiologists were always referred for further analysis. During 2 years follow-up, we collected the radiology reports, surgical correspondence and pathology reports of all referred women and interval breast cancers.

\section{Results}

Respectively 44,491 and 42,996 screens had been read either in a blinded or non-blinded fashion. Recall rate $(3.3 \%$ versus $2.8 \%, P<0.001)$ and false positive rate $(2.6 \%$ versus $2.2 \%, P=0.002)$ were significantly higher at blinded double reading whereas the cancer detection rate per 1,000 screens ( 7.4 versus $6.5, P=0.14$ ) and positive predictive value of recall ( $22 \%$ versus $23 \%, P=0.51$ ) were comparable. Blinded double reading resulted in a significantly higher program sensitivity ( $83 \%$ versus $76 \%, P=0.01$ ). Per 1000 screened women, blinded double reading would yield 0.9 more screen detected cancers and 0.6 less interval cancers than non-blinded double reading, at the expense of 4.4 more recalls.

\section{Conclusion}

We advocate the use of blinded double reading in order to achieve a better program sensitivity, at the expense of an increased recall rate and false positive recall rate. 


\section{Introduction}

In the last two decades, regional and nation-wide screening mammography programs have been implemented in many Western countries. ${ }^{1}$ The reduction in breast cancer mortality is attributed to the combination of earlier breast cancer detection through mammography screening and substantial improvements in breast cancer treatment. ${ }^{2-5}$

Assessment of screening mammograms can be performed in several ways, e.g. by single radiologist reading, single reading with computer aided detection (CAD), or double reading. Double reading by two specialized breast screening radiologists is considered to be the standard of reference in Europe. ${ }^{6}$ This reading strategy significantly increases the cancer detection rate when compared to single reading. ${ }^{7-9}$ Assessment by CAD or screening mammography technologists, in addition to radiologist single reading, may be considered when radiologist double reading is not feasible due to a shortage of trained and experienced radiologists.

Since 1995, the Dutch nationwide breast cancer screening program provides biennial screening mammography for women aged $50-75$ years. The mammograms are always read by two certified screening radiologists, mostly in a non-blinded fashion. In case of a discrepant reading, the two radiologists may discuss the case together to reach consensus about recall, a third reader may be added for arbitration, or the woman may routinely be referred without consensus reading or arbitration. ${ }^{10}$

In our screening region in the Southern Netherlands, digital mammography was introduced in 2009 and the transition from screen film mammography (SFM) to fullfield digital mammography (FFDM) was completed at the beginning of 2010. Our screening region has a close collaboration with the National Expert and Training Centre for Breast Cancer Screening, the Comprehensive Cancer Centre South (IKZ)/Eindhoven Cancer Registry as well as the breast cancer departments in community hospitals. With the introduction of FFDM it became technically possible to perform blinded double reading instead of non-blinded double reading. The purpose of the current study was to prospectively determine screening mammography outcome at blinded versus nonblinded double reading.

\section{Methods}

\section{Study population}

We included a consecutive series of 87,487 full-field digital screening mammograms performed between July 1, 2009 and July 1, 2011. The screens were obtained at three specialized screening units in a southern biennial screening mammography region of the Netherlands (BOZ, Bevolkings Onderzoek Zuid). Women participating in the nationwide Dutch screening program are routinely asked to give permission for the use of their data for scientific purposes. Two women screened at our units did not give this 
permission and they were excluded. This study was performed within the national permit for breast cancer screening, which is issued by the secretary of health after advice of the national health counsel. The study did not require a special permit according to the Dutch Law on Population-based screening as both blinded and nonblinded double reading were considered "standard of care" at the time of the study.

\section{Screening procedure and recall}

Details of the nationwide breast cancer screening program have been described previously. ${ }^{10}$ Mammograms were acquired with a Lorad Selenia FFDM system (Hologic Inc, Danbury, CT), with a $70 \mu \mathrm{m}$ pixel size and a $232 \times 286 \mathrm{~mm}$ field of view. The mammographic examinations were double read by a team of 12 certified screening radiologists. Each radiologist evaluated at least 6,000 screening mammograms yearly. Prior screening mammograms were always available for comparison at the time of a subsequent screening round. To facilitate comparison of subsequent FFDM screens with prior SFM screens, the most recent screen-film mammograms were digitized by fusion equipments designed for mammography (DigitalNow;R2/Hologic).

Screening mammograms were double read in either a blinded $\left(2^{\text {nd }}\right.$ reader was not informed about the $1^{\text {st }}$ reader's decision) or non-blinded fashion $\left(2^{\text {nd }}\right.$ reader was informed about the $1^{\text {st }}$ reader's decision). This reading strategy was alternated on a monthly basis. Women with discrepant readings between the two screening radiologists, at blinded and non-blinded double reading, were always referred for further analysis.

Each screen was classified according to the Breast Imaging Reporting and Data System (BI-RADS) as BI-RADS category $0,1,2,4$ or $5 .{ }^{11}$ Women with a screening BI-RADS 0,4 or 5 were referred to a dedicated breast unit. At screening, mammographic abnormalities were classified according to the following categories: suspicious mass, suspicious microcalcifications, suspicious mass in combination with microcalcifications, architectural distortion, asymmetry, or other abnormality.

\section{Diagnostic work-up and follow-up of referred women}

A total of 15 regional and university hospitals were involved in the assessment of screen-positive women. After physical examination by the surgeon, additional mammographic views were obtained if necessary. At diagnostic workup, radiologists again classified the radiologic findings according to the BI-RADS. ${ }^{11}$ Dependent on the findings at physical examination and the diagnostic workup protocols and the various hospitals, further diagnostic evaluation could include breast ultrasonography, magnetic resonance mammography, percutaneous fine-needle aspiration biopsy (FNAB), core needle biopsy (CNB) or open excision biopsy. BI-RADS 4 and 5 lesions were routinely biopsied, whereas BI-RADS 3 lesions were either biopsied or followed-up.

During a follow-up period of at least two years (until the next biennial screening round), we collected data on diagnostic imaging procedures, clinical data, biopsy 
specimen and surgical procedures of all referred women. Breast cancers were divided into ductal carcinoma in situ (DCIS) and invasive cancer. Lobular carcinoma in situ was left out of consideration. Data on TNM (tumor-node-metastases) classification ${ }^{12}$ were collected. Sentinel nodes were classified negative if they harbored isolated tumor cells or sub-micrometastases $\left(<0.2 \mathrm{~mm}, \mathrm{NO}_{(\text {itc })}\right)$ and were considered positive if they contained micrometastases $\left(0.2-2 \mathrm{~mm}, \mathrm{~N} 1_{(\mathrm{mi})}\right)$ or macrometastases ( $>2 \mathrm{~mm}, \mathrm{N1}-3$ ).

\section{Detection of interval cancers}

Interval cancers are breast cancers diagnosed after a negative screening mammography (i.e., screening without a recommendation for recall), and before the next biennial screening examination. Calculation of the program sensitivity of the screening strategies requires identification of all interval cancers.

We traced interval cancers, by using all available information in addition to the usual follow-up procedure, including:

- All radiotherapy reports that were received from the regional radiotherapy institutes concerning women who underwent radiotherapy for breast malignancy and who had participated in the screening program;

- Inquiry about pathology specimens at the various regional pathology laboratories, some months after any hospital had requested the screening mammograms of a woman who had not been referred for further analysis;

- The pathology records that were obtained if a woman cancelled a call for subsequent screening because breast cancer had been diagnosed after a previous negative screen;

- The occasional reports on interval cancers provided by general practitioners or medical specialists to the screening centre.

\section{Statistical analysis}

The main outcome measures were recall rate (recalls per 100 screens), cancer detection rate (CDR, screen detected cancers per 1000 screens), false positive rate (FPR, false positive recalls per 1000 screens), interval cancer rate (ICR, interval cancers per 1000 screens), program sensitivity and positive predictive value of recall (PPV, screen detected cancers per 100 recalls). Screening program sensitivity was calculated by determination of the proportion of screen-detected cancers out of all breast cancers (screen-detected and interval cancers) diagnosed during the 2-year screening interval. The BI-RADS at recall (highest BI-RADS of two readers) of the two reading strategies were compared for all recalls. For this calculation BI-RADS at recall were divided in $\mathrm{BI}-$ RADS 0 and BI-RADS 4 or 5.

A logistic regression model was used to investigate the association between reading strategy and recall rate, cancer detection rate, false positive rate, interval cancer rate, positive predictive value, program sensitivity, and BI-RADS at recall. Because a pair of radiologists reads many screening examinations, the classifications 
within such a pair of radiologists will be correlated. To account for this dependency of the data, a generalized estimating equation approach was used to estimate the $p$ values and percentages for the comparison of the reading strategies using the logistic regression model. A Chi square and fisher exact test were used to test differences in the distribution and rate per 1000 screened women of tumor and mammographic characteristics of the two reading strategies.

Statistics were performed using Statistical Package for Social Science 17.0 (SPSS Inc. Chicago, IL) and SAS ${ }^{\circledR}$ 9.2. A $P$-value of less than 0.05 was considered to indicate statistical significance.

\section{Results}

\section{Overall screening results}

Respectively 44,491 and 42,996 screens had been read, either in a blinded or nonblinded fashion. The mean age of both study groups was similar (both 59 years, $95 \% \mathrm{Cl}$ 59-60). The model-estimated results are listed in Table 4.1.1. The recall rate (3.3\% versus $2.9 \%, P=0.002)$, as well as the proportion of discrepant readings among referred women ( $57 \%$ versus $30 \%, P<0.001$ ), was significantly higher at blinded double reading than at non-blinded reading. No differences were found between blinded and nonblinded reading in the BI-RADS classification at recall $(P=0.955)$. There were significantly more false positive recalls at blinded double reading $(2.6 \%$ versus $2.2 \%, P=0.002)$. The cancer detection rate per 1,000 screened women and the positive predictive value of recall were comparable for both reading strategies, respectively $7.4 \%$ o versus $6.5 \%$ o $(P=0.139)$ and $22 \%$ versus $23 \%(P=0.507)$.

We traced a total of 158 interval cancers, of which 66 (1.5 per 1,000 screens) had been diagnosed after blinded double reading and 92 (2.1 per 1,000 screens) after nonblinded double reading, and this difference was statistically significant $(P=0.017)$. The program sensitivity was significantly higher at blinded double reading $(83 \%$ versus $76 \%$, $P=0.003)$. 
Table 4.1.1 Overall results of blinded versus non-blinded double reading of screening mammograms

\begin{tabular}{l|ccc|} 
& $\begin{array}{c}\text { Blinded double reading } \\
(\mathrm{n}=44,4910)^{*}\end{array}$ & $\begin{array}{c}\text { Non-blinded double } \\
\text { reading } \\
(\mathrm{n}=42,996)^{*}\end{array}$ & $P$-value* \\
\hline Recall rate, $\mathrm{n}(\%)$ & $1474(3.3)$ & $1235(2.9)$ & 0.002 \\
\hline Proportion of BI-RADS 4 or 5, $\mathrm{n}(\%)$ & $997(66.7)$ & $841(66.9)$ & 0.955 \\
\hline Proportion of discrepant readings, $\mathrm{n}(\%)$ & $842(56.7)$ & $359(29.8)$ & $<0.001$ \\
\hline False positive recalls per 1000 screens, $\mathrm{n}(\%)$ & $1149(25.8)$ & $951(22.1)$ & 0.002 \\
Cancer detection rate per 1,000 screens, $\mathrm{n}(\%)$ & $325(7.4)$ & $284(6.5)$ & 0.139 \\
Positive predictive value of recall, \% & 22.1 & 23.1 & 0.507 \\
Interval cancer rate per 1,000 screens, $\mathrm{n}(\%)$ & $66(1.5)$ & $92(2.1)$ & 0.017 \\
Program sensitivity, \% & 83.1 & 75.5 & 0.003
\end{tabular}

Cl confidence interval; BI-RADS Breast Imaging Reporting And Data System. *Percentages and P-values base on logistic regression analysis

\section{Mammographic abnormalities, tumor characteristics of screen-detected cancers}

The mammographic abnormalities of screen-detected cancers were similar at the two reading strategies, a suspicious mass was the most frequently encountered reason of recall (about $50 \%$ of recalls), followed by suspicious microcalcifications (Table 4.1.2).

We observed no differences in the proportion and detection per 1000 screened women of DCIS ( $23 \%$ versus $26 \%$ ), smaller (T1a-c) tumors ( $81 \%$ versus $80 \%$ ), or invasive cancers with axillary lymph node metastasis $(17 \%$ versus $22 \%, P=0.177)$ between blinded and non-blinded double reading (Table 4.1.2). The histological subtypes and tumor grading of DCIS and invasive cancers were also comparable for both reading strategies. The majority of invasive cancers, about $80 \%$, were of the ductal type at both reading strategies, followed by invasive lobular cancer (10\%). Equal proportions of DCIS (about $1 / 3$ low grade, $1 / 3$ intermediate grade, and $1 / 3$ high grade) were found, and just over $50 \%$ of invasive cancers were Nottingham grade I, both at blinded and non-blinded double reading. Finally, the proportions and rates of estrogen, progesterone or Her2/Neu receptor positive invasive cancers were also comparable, as well as the proportion of triple negative invasive cancers (Table 4.1.2).

\section{Tumor characteristics of interval cancer}

The proportion of DCIS, invasive cancers and tumor grading of interval cancers were similar at the two reading strategies (Table 4.1.3). After non-blinded double reading, significantly more invasive interval cancers were detected per 1000 screened women, compared to blinded double reading $(+0.7)$. These invasive cancers were more often T1a-c cancers $(+0.5)$, lymph node positive cancers $(+0.5)$, Nottingham grade 1 cancers $(+0.4)$, estrogen- or progesterone-receptor positive cancer (both +0.6$)$, Her2/Neunegative cancers $(+0.7)$ and not triple-negative cancers (+0.6) (Table 4.1.3). 
Table 4.1.2: Tumor characteristics and mammographic abnormalities of screen-detected cancers

\begin{tabular}{|c|c|c|c|c|c|c|c|c|}
\hline & \multicolumn{5}{|c|}{ Proportion } & \multicolumn{3}{|c|}{$\begin{array}{l}\text { Rate (per } 1000 \text { screened } \\
\text { women) }\end{array}$} \\
\hline & \multicolumn{2}{|c|}{$\begin{array}{c}\text { BDR } \\
n=325\end{array}$} & \multicolumn{2}{|c|}{$\begin{array}{l}\text { NBDR } \\
n=284\end{array}$} & \multirow[t]{2}{*}{$P$-value } & \multirow[t]{2}{*}{ BDR } & \multirow[t]{2}{*}{ NBDR } & \multirow[t]{2}{*}{$P$-value } \\
\hline & $\mathrm{n}$ & $\%$ & $\mathrm{n}$ & $\%$ & & & & \\
\hline Mammographic abnormality & & & & & 0.825 & & & \\
\hline Mass & 173 & 53.2 & 146 & 52.5 & & 3.9 & 3.4 & 0.227 \\
\hline Microcalcifications & 98 & 30.2 & 80 & 28.2 & & 2.2 & 1.9 & 0.262 \\
\hline Mass with microcalcifications & 34 & 10.5 & 36 & 12.7 & & 0.8 & 0.8 & 0.702 \\
\hline Asymmetry & 1 & 0.3 & 1 & 0.4 & & 0.0 & 0.0 & 1.000 \\
\hline Architectural distortion & 19 & 5.8 & 21 & 7.4 & & 0.4 & 0.5 & 0.671 \\
\hline Type of screen-detected cancer & & & & & 0.507 & & & \\
\hline DCIS & 76 & 23.4 & 73 & 25.7 & & 1.7 & 1.7 & 0.970 \\
\hline Invasive & 249 & 76.6 & 211 & 74.3 & & 5.6 & 4.9 & 0.159 \\
\hline DCIS & & & & & 0.870 & & & \\
\hline Low grade & 24 & 31.6 & 24 & 32.9 & & 0.5 & 0.6 & 0.906 \\
\hline Intermediate grade & 27 & 35.5 & 23 & 31.5 & & 0.6 & 0.5 & 0.656 \\
\hline High grade & 25 & 32.9 & 26 & 35.6 & & 0.6 & 0.6 & 0.793 \\
\hline Histologic type of invasive cancers & & & & & 0.780 & & & \\
\hline Ductal & 197 & 79.1 & 172 & 81.5 & & 4.4 & 4.0 & 0.329 \\
\hline Lobular & 27 & 10.8 & 18 & 8.5 & & 0.6 & 0.4 & 0.220 \\
\hline Mixed ductal/lobular & 13 & 5.2 & 9 & 4.3 & & 0.3 & 0.2 & 0.440 \\
\hline Other & 12 & 4.8 & 12 & 5.7 & & 0.3 & 0.3 & 0.993 \\
\hline Size of invasive cancers & & & & & 0.982 & & & \\
\hline T1a-c (1-20 mm) & 202 & 81.1 & 171 & 81.0 & & 4.5 & 4.0 & 0.201 \\
\hline $\mathrm{T} 2(>20 \mathrm{~mm})$ & 47 & 18.9 & 40 & 19.0 & & 1.1 & 0.9 & 0.554 \\
\hline Lymph node status of invasive cancers & & & & & 0.177 & & & \\
\hline Positive & 43 & 17.3 & 47 & 22.3 & & 1.0 & 1.1 & 0.559 \\
\hline Negative & 206 & 82.7 & 164 & 77.7 & & 4.6 & 3.8 & 0.063 \\
\hline Nottingham grade of invasive cancers & & & & & 0.732 & & & \\
\hline Nottingham I & 125 & 50.2 & 110 & 52.1 & & 2.8 & 2.6 & 0.473 \\
\hline Nottingham II & 99 & 39.8 & 86 & 40.8 & & 2.2 & 2.3 & 0.469 \\
\hline Nottingham III & 21 & 8.4 & 13 & 6.2 & & 0.5 & 0.3 & 0.203 \\
\hline Unknown & 4 & 1.6 & 2 & 0.9 & & 0.1 & 0.0 & 0.668 \\
\hline Estrogen receptor of invasive cancers & & & & & 0.686 & & & \\
\hline Positive & 224 & 90.0 & 190 & 90.0 & & 5.0 & 4.4 & 0.185 \\
\hline Negative & 22 & 8.8 & 20 & 9.5 & & 0.5 & 0.5 & 0.843 \\
\hline Unknown & 3 & 1.2 & 1 & 0.5 & & 0.1 & 0.0 & 0.625 \\
\hline Progesterone receptor of invasive cancers & & & & & 0.302 & & & \\
\hline Positive & 180 & 72.3 & 142 & 67.3 & & 4.0 & 3.3 & 0.070 \\
\hline Negative & 66 & 26.5 & 68 & 32.2 & & 1.5 & 1.6 & 0.711 \\
\hline Unknown & 3 & 1.2 & 1 & 0.5 & & 0.1 & 0.0 & 0.625 \\
\hline Her2/Neu receptor of invasive cancers & & & & & 0.225 & & & \\
\hline Positive & 17 & 6.8 & 24 & 11.4 & & 0.4 & 0.6 & 0.229 \\
\hline Negative & 229 & 92.0 & 184 & 87.2 & & 5.1 & 4.3 & 0.061 \\
\hline Unknown & 3 & 1.2 & 3 & 1.4 & & 0.1 & 0.1 & 1.000 \\
\hline Triple-receptor negative & & & & & 0.978 & & & \\
\hline Yes & 18 & 7.2 & 15 & 6.7 & & 0.4 & 0.3 & 0.671 \\
\hline No & 228 & 91.6 & 193 & 91.9 & & 5.1 & 4.5 & 0.174 \\
\hline Unknown & 3 & 1.2 & 3 & 1.4 & & 0.1 & 0.1 & 1.000 \\
\hline
\end{tabular}

BDR blinded double reading; NBDR non-blinded double reading; DCIS ductal carcinoma in situ 
Table 4.1.3 Tumor characteristics of interval cancers

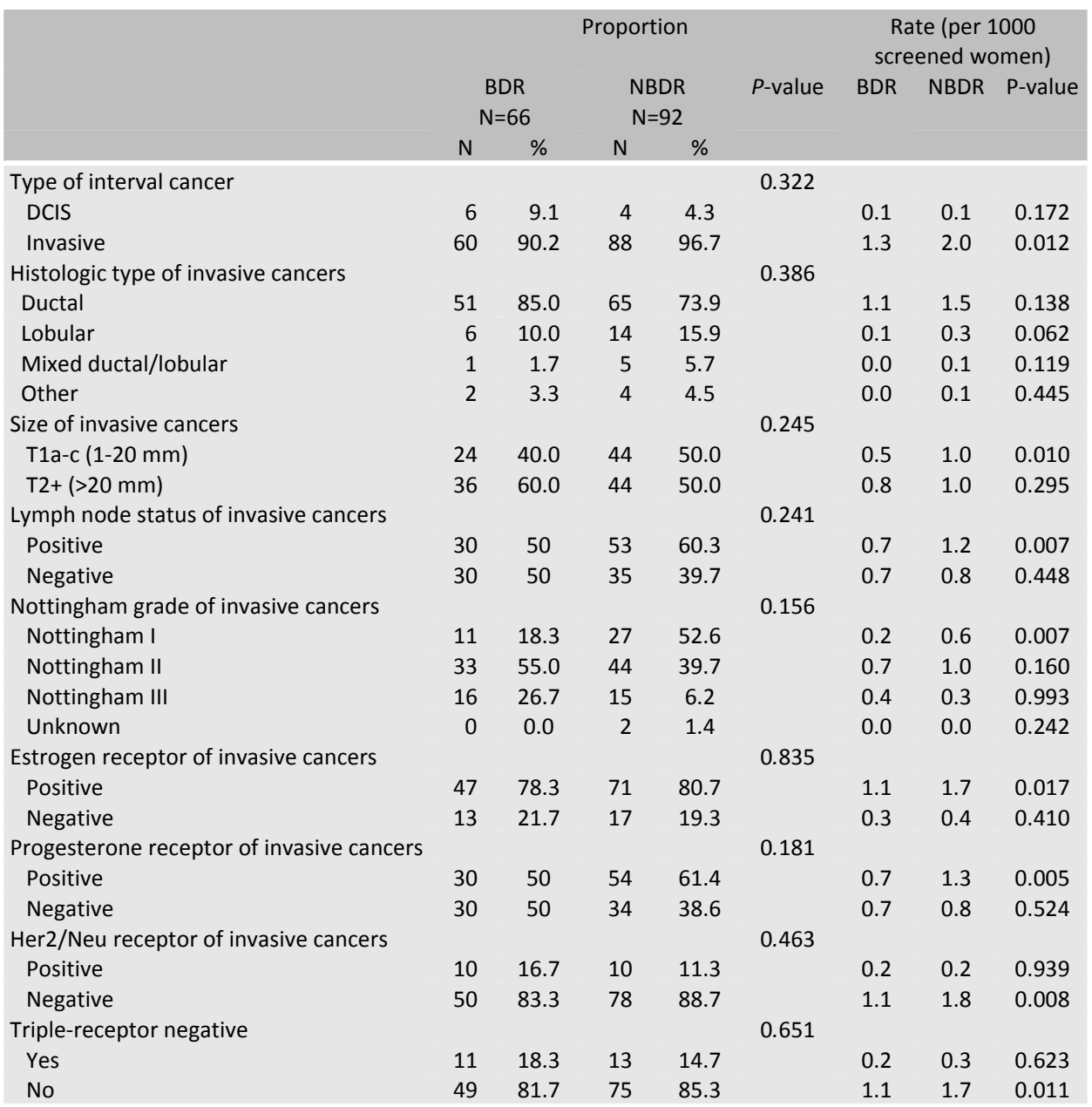

BDR blinded double reading; NBDR non-blinded double reading; DCIS ductal carcinoma in situ

\section{Discussion}

To our knowledge, this is the first study reporting on the outcome of blinded versus non-blinded double reading at screening mammography. Blinded double reading resulted in significantly higher program sensitivity, at the expense of an increased recall rate, a higher proportion of discrepant readings among referred women and an increased false positive recall rate. Per 1000 screened women, blinded double reading would yield 0.9 more screen detected cancers and 0.6 less interval cancers than nonblinded double reading, at the expense of 4.4 more recalls. 
Reading of screening mammograms can be performed in several ways. Many programs use non-blinded, or independent, double reading by two radiologists. We found that blinded double reading yielded a significantly better program sensitivity than non-blinded double reading, as well as a $14 \%$ higher (7.4 versus 6.5), although non-significant, cancer detection rate. The tumor and mammographic characteristics of screen-detected cancers were comparable for both reading strategies. After nonblinded double reading, significantly more invasive interval cancers were detected per 1000 screened women, compared to blinded double reading. These invasive cancers were more often, smaller T1a-c cancers, lymph node positive cancers, Nottingham grade 1 cancers, estrogen- or progesterone-receptor positive cancer, Her2/Neunegative cancers and not triple-negative cancers.

Several issues should be considered prior to adoption of blinded double reading. Blinded double reading almost doubled the proportion of discrepant readings among referred women in our study, which resulted in a significantly higher recall rate at blinded double reading. The Dutch breast screening program is characterized by low recall rates, and our observed recall rate of $3.3 \%$ at blinded double reading is still low compared to the recall rates of screening programs elsewhere. ${ }^{13}$ Otten et al. ${ }^{14}$ calculated that a recall rate of more than $4 \%$ has no additional positive effect on the cancer detection rate in the Netherlands. Although our recall rate at blinded double reading was still lower than this $4 \%$, an increased recall rate may be unfavorable for screening programs already characterized by a significantly higher baseline recall rate at non-blinded double reading. Furthermore, the higher false positive recall rate at blinded double reading increases work up costs and it is well known that a false positive recall may cause temporary anxiety and psychological distress. ${ }^{15-17}$ Although there are conflicting reports whether or not a false positive test changes a woman's future screening adherence, ${ }^{18-21}$ it is obvious that the amount of false positive recalls should be kept as low as possible.

Another potentially harmful effect of screening is over-diagnosis, i.e diagnosis of breast cancers that, if left undiscovered, would never become clinically evident and, thus, would never become lethal. Estimates of over-diagnosis vary greatly among studies, ranging from $1 \%$ to $50 \% .^{22-25}$ The incidence of DCIS increased markedly in southern Netherlands since the introduction of screening mammography in $1992^{26}$ and a recent Dutch study reported a more than doubling of the DCIS detection rate at FFDM compared to SFM. ${ }^{27}$ As in many other screening programs, ${ }^{28}$ we found that, one out of four screen detected cancers comprised DCIS, both at blinded and non-blinded double reading. Although DCIS can be a precursor of invasive breast cancer, not all DCIS lesions will progress to invasive carcinoma. ${ }^{29}$ Currently, it is not clear whether or not DCIS should be treated routinely by breast conserving surgery in combination with radiotherapy or, in the case of larger DCIS lesions, by mastectomy. In the Netherlands, a randomized trial on the management of low risk DCIS will start in the near future, comparing standard therapy with active surveillance. 
During the study period 391 cancers (325 SDC and 66 interval cancers) were detected among 44,491 screens at blinded double reading, resulting in 8.78 cancers per 1000 screened women. At non-blinded double reading 376 cancers (284 SDC and 92 interval cancers) were detected among 42,996 screens, resulting in 8.74 cancers per 1000 screened women. Further, tumor characteristics of SCD were comparable for both screening strategies. These findings suggest that the amount of over-diagnosis does not significantly differ at blinded and non-blinded double reading. $(P=0.945)$

In the current study, women with a discrepant reading between two screening radiologists were always referred for further analysis. Alternatively, the two radiologists may try to reach consensus in case of a discrepant reading, or arbitration by a third radiologist may be considered. In case of a discrepant reading, one of these alternative reading strategies may lower the amount of false positive recalls in screening programs characterized by a higher baseline recall rate. ${ }^{10,30}$ This is a subject of future research.

New Technologies accompanying digital mammography, like Computer-aideddetection (CAD) or tomosynthesis, could diminish the need for double blind reading. Digital mammography Computer-aided-detection (CAD) has been available for over ten years and has extensively been evaluated at screen-film screening mammography programs. However, its contribution to routine screening remains a question of debate. In a systematic review, Azavedo et al. concluded that there is insufficient evidence to determine whether CAD could replace a second reader. ${ }^{31}$ On the other hand, recent studies have shown that the addition of breast tomosynthesis to digital mammography improves the cancer detection rate in mammography screening and may reduce false positive recalls. ${ }^{32,33}$ These results are promising, however, a recent published paper of Houssami et al. ${ }^{34}$ showed that although double-reading had a relatively modest additional detection effect at 2D/3D mammography, its effect was not negated.

\section{Our study has certain limitations}

Every other month, screening mammograms were double read, either in a non-blinded or blinded fashion. The study design could have benefited from randomization of screened women among the two screening strategies. Unfortunately, this randomization was not feasible in our daily practice. However, we expect that our quasi-randomized model (screening strategies were altered on a monthly basis) will not result in different outcomes than a true randomization.

At the time of the study, a working and validated linkage between the Dutch National comprehensive Cancer Centre and the Screening Information System was not available yet. Therefore we traced interval cancers, by using all available information in addition to the usual follow-up procedure to detect interval cancers, as mentioned in the method section. We are quite confident that this method is reliable. The linkage of registries in the near future will provide even more opportunities for quality control.

Further, although all radiologists had more than five years of experience with working in a digital radiology environment, including digital mammography, they had little experience with FFDM screening at the start of the study. Nevertheless, it is 
unlikely that our results have been influenced by a learning effect, as the recall rate, cancer detection rate and PPV of recall did not change during the FFDM screening period. $^{35}$

Our study does not provide information on the cost-effectiveness of blinded double reading. For example, we did not record the time needed by radiologists for the assessment of screening mammograms, either in a blinded or non-blinded fashion. One may suggest that screening in a blinded fashion increases screening time, as the second reader 'lacks' the first reader's opinion. On the other hand, reading of screening mammograms can be speeded up if the second reader does not have to take the other reader's opinion into account. Moreover, the higher recall rate at blinded double reading increases the recall of lesions that turn out to be benign and thus increases work up costs. ${ }^{36}$ An additional cost effectiveness analysis will be necessary to determine whether blinded double reading, combined with recall on the authority of one radiologist without consensus reading, should become the nationwide standard in the Netherlands.

Finally, the purpose of this study was to prospectively determine screening mammography outcomes at blinded and non-blinded double reading. Our main outcome measures were recall rate, CDR, program sensitivity, false positive rate, interval cancer rate and positive predictive value of recall. The best way to determine the comparative effectiveness of screening procedures is by breast cancer mortality. This was not yet achievable, due to the short follow-up period (two years) and is therefore a subject of future research. Nevertheless, significant more invasive interval cancers were detected after non-blinded double reading compared to blinded double reading. Interval cancers have a worse survival than SDC. ${ }^{37}$ This assumption suggests that blinded double reading can potentially save more lives than non-blinded double reading.

In summary, we advocate the use of blinded double reading in order to achieve a better program sensitivity, at the expense of an increased recall rate and false positive recall rate. 


\section{References}

1 Dowling EC, Klabunde C, Patnick J, Ballard-Barbash R. Breast and cervical cancer screening programme implementation in 16 countries. J Med Screen 2010;17:139-146.

2 van Schoor G, Moss SM, Otten JD et al. Increasingly strong reduction in breast cancer mortality due to screening. Br J Cancer 2011;104:910-914.

3 Burton RC, Bell RJ, Thiagarajah G, Stevenson C. Adjuvant therapy, not mammographic screening, accounts for most of the observed breast cancer specific mortality reductions in Australian women since the national screening program began in 1991. Breast Cancer Res Treat 2012;131:949-955.

4 Mandelblatt J, van Ravesteyn N, Schechter C et al. Which strategies reduce breast cancer mortality most? Collaborative modeling of optimal screening, treatment, and obesity prevention. Cancer 2013;119:2541-2548.

5 Weedon-Fekjaer H, Romundstad PR, Vatten L. Modern mammography screening and breast cancer mortality: population study. BMJ 2014;348:g3701.

6 Perry N, Broeders M, de Wolf C, Tornberg S, Holland R, von Karsa L. European guidelines for quality assurance in breast cancer screening and diagnosis. Fourth edition--summary document. Ann Oncol 2008;19:614-622.

7 Harvey SC, Geller B, Oppenheimer RG, Pinet M, Riddell L, Garra B. Increase in cancer detection and recall rates with independent double interpretation of screening mammography. AJR Am J Roentgenol 2003;180:1461-1467.

8 Thurfjell EL, Lernevall KA, Taube AA. Benefit of independent double reading in a population-based mammography screening program. Radiology 1994;191:241-244.

9 Brown J, Bryan S, Warren R. Mammography screening: an incremental cost effectiveness analysis of double versus single reading of mammograms. BMJ 1996;312:809-812.

10 Duijm LE, Groenewoud JH, Hendriks JH, de Koning HJ. Independent double reading of screening mammograms in The Netherlands: effect of arbitration following reader disagreements. Radiology 2004;231:564-570.

11 Liberman L, Menell JH. Breast imaging reporting and data system (BI-RADS). Radiol Clin North Am 2002;40:409-430, v.

12 Sobin LH, Wittekind C. TNM Classification of Malignant Tumours. Wiley-Liss, New York. 2002.

13 Smith-Bindman R, Chu PW, Miglioretti DL et al. Comparison of screening mammography in the United States and the United kingdom. JAMA 2003;290:2129-2137.

14 Otten JD, Karssemeijer N, Hendriks JH et al. Effect of recall rate on earlier screen detection of breast cancers based on the Dutch performance indicators. J Natl Cancer Inst 2005;97:748-754.

15 Bond $\mathrm{M}$, Pavey $\mathrm{T}$, Welch $\mathrm{K}$ et al. Psychological consequences of false-positive screening mammograms in the UK. Evid Based Med 2013;18:54-61.

16 Brewer NT, Salz T, Lillie SE. Systematic review: the long-term effects of false-positive mammograms. Ann Intern Med 2007; 146:502-510.

17 van der Steeg AF, Keyzer-Dekker CM, De Vries J, Roukema JA. Effect of abnormal screening mammogram on quality of life. Br J Surg 2011;98:537-542.

18 Roman R, Sala M, De La Vega M et al. Effect of false-positives and women's characteristics on long-term adherence to breast cancer screening. Breast Cancer Res Treat 2011;130:543-552.

19 Pinckney RG, Geller BM, Burman M, Littenberg B. Effect of false-positive mammograms on return for subsequent screening mammography. Am J Med 2003;114:120-125.

20 McCann J, Stockton D, Godward S. Impact of false-positive mammography on subsequent screening attendance and risk of cancer. Breast Cancer Res 2002;4:R11.

21 Lampic C, Thurfjell E, Sjoden PO. The influence of a false-positive mammogram on a woman's subsequent behaviour for detecting breast cancer. Eur J Cancer 2003;39:1730-1737.

22 Puliti D, Duffy SW, Miccinesi G et al. Overdiagnosis in mammographic screening for breast cancer in Europe: a literature review. J Med Screen 2012;19 Suppl 1:42-56.

23 Miller AB, Wall C, Baines CJ, Sun P, To T, Narod SA. Twenty five year follow-up for breast cancer incidence and mortality of the Canadian National Breast Screening Study: randomised screening trial. BMJ 2014;348:g366.

24 Jorgensen KJ, Keen JD, Gotzsche PC. Is mammographic screening justifiable considering its substantial overdiagnosis rate and minor effect on mortality? Radiology 2011;260:621-627. 
25 Kopans DB, Smith RA, Duffy SW. Mammographic screening and "overdiagnosis". Radiology 2011;260:616-620.

26 van Steenbergen LN, Voogd AC, Roukema JA et al. Screening caused rising incidence rates of ductal carcinoma in situ of the breast. Breast Cancer Res Treat 2009;115:181-183.

27 de Gelder R, Heijnsdijk EA, van Ravesteyn NT, Fracheboud J, Draisma G, de Koning HJ. Interpreting overdiagnosis estimates in population-based mammography screening. Epidemiol Rev 2011;33:111121.

28 Bundred N, Dixon JM. Carcinoma in situ. BMJ 2013;347:f3289.

29 Bijker N, Donker M, Wesseling J, den Heeten GJ, Rutgers EJ. Is DCIS breast cancer, and how do I treat it? Curr Treat Options Oncol 2013;14:75-87.

30 Caumo F, Brunelli S, Tosi $\mathrm{E}$ et al. On the role of arbitration of discordant double readings of screening mammography: experience from two Italian programmes. Radiol Med 2011;116:84-91.

31 Azavedo E, Zackrisson S, Mejare I, Heibert Arnlind M. Is single reading with computer-aided detection (CAD) as good as double reading in mammography screening? A systematic review. BMC Med Imaging 2012;12:22.

32 Ciatto S, Houssami N, Bernardi D et al. Integration of 3D digital mammography with tomosynthesis for population breast-cancer screening (STORM): a prospective comparison study. Lancet Oncol 2013;14:583-589.

33 Friedewald SM, Rafferty EA, Rose SL et al. Breast cancer screening using tomosynthesis in combination with digital mammography. JAMA 2014;311:2499-2507.

34 Houssami N, Macaskill P, Bernardi D et al. Breast screening using 2D-mammography or integrating digital breast tomosynthesis (3D-mammography) for single-reading or double-reading--evidence to guide future screening strategies. Eur J Cancer 2014;50:1799-1807.

35 Nederend J, Duijm LE, Louwman MW, Groenewoud JH, Donkers-van Rossum AB, Voogd AC. Impact of transition from analog screening mammography to digital screening mammography on screening outcome in The Netherlands: a population-based study. Ann Oncol 2012;23:3098-3103.

36 Duijm LE, Groenewoud JH, Fracheboud J et al. Utilization and cost of diagnostic imaging and biopsies following positive screening mammography in the southern breast cancer screening region of the Netherlands, 2000-2005. Eur Radiol 2008;18:2390-2397.

37 Fong Y, Evans J, Brook D, Kenkre J, Jarvis P, Gower Thomas K. The incidence and 10-year survival of interval breast cancers in Wales. Clin Radiol 2014;69:e168-172. 


\section{Chapter 4.2}

Comparison of the diagnostic workup of women recalled at non-blinded or blinded double reading in a population-based screening mammography program in the South of The Netherlands

Weber RJP, Klompenhouwer EG, Voogd AC, Strobbe LJA, Broeders MJM, Duijm LEM Br J Cancer: accepted July 2015 


\section{Abstract}

\section{Background}

To determine whether recalled women experience differences in diagnostic workup at nonblinded or blinded double reading of screening mammograms.

\section{Methods}

We included a consecutive series of respectively 42.996 and 44.491 screens, double read either in a non-blinded or blinded fashion between 2009 and 2011. This reading strategy was alternated on a monthly basis.

\section{Results}

The overall ultrasound guided core needle biopsy (CNB) rate and stereotactic core needle biopsy $(\mathrm{SCNB})$ rate per 1000 screens were higher at blinded than at non-blinded reading (7.5 versus 6.0, $P=0.008$ and 8.1 versus 6.6, $P=0.009$ ). Among women with benign workup, these rates were higher at blinded reading ( 2.6 versus $1.4, P<0.001$ and 5.9 versus $4.7, P=0.013$ ). The benign biopsy rates were higher at blinded double reading $(P<0.001)$, whereas the positive predictive value of biopsy (PPV) did not differ $(P=0.103)$.

\section{Conclusion}

Blinded double reading results in higher overall CNB and SCNB rates than non-blinded double reading, as well as a higher benign biopsy rate. 


\section{Introduction}

Reduction in breast cancer mortality is attributed to the combination of earlier breast cancer detection through mammography screening and substantial improvements in breast cancer treatment. ${ }^{1-3}$

Screening mammograms can be assessed in several ways, e.g. by single reading or double reading by certified screening radiologists. European guidelines consider double reading by radiologists as the gold standard for the assessment of screening mammograms as this screening strategy significantly increases the cancer detection rate when compared to single reading. ${ }^{4,5}$ In most programs, including the Dutch nationwide breast cancer screening program, independent double reading rather than blinded double reading is used for the assessment of screening mammograms. We recently compared these two reading strategies and found that blinded double reading increased the sensitivity of breast cancer detection, at the expense of an increased referral rate and decreased positive predictive value of referral. ${ }^{6}$ However, the influence of replacement of non-blinded by blinded double reading on the use of diagnostic resources at the workup of screen-positive women is unknown. We therefore compared the utilization of breast imaging and biopsy techniques in women recalled at blinded or non-blinded double reading in a population-based screening mammography program in the South of the Netherlands.

\section{Materials and methods}

\section{Study population}

We used the information of respectively 42996 and 44491 full-field digital screens that had been double read, either in a non-blinded or blinded fashion between July 2009 and July $2011 .^{6}$

\section{Screening procedure and referral}

Details of the nationwide and local breast cancer screening program have been described previously. ${ }^{6-8}$ In brief, free biennial screening mammography is offered to women aged $50-75$ years. Before each screening examination, the woman completes a short questionnaire with questions about any previous breast surgery or breast malignancy and the use of hormone replacement therapy. Screening mammograms were double read in either a blinded $\left(2^{\text {nd }}\right.$ reader was not informed about the $1^{\text {st }}$ reader's decision) or non-blinded $\left(2^{\text {nd }}\right.$ reader was informed about the $1^{\text {st }}$ reader's decision). This reading strategy was alternated on a monthly basis. Mammograms of previous screening rounds were always available for comparison.

Women with a screening Breast Imaging Reporting and Data System (BI-RADS) category 0,4 or 5 were recalled to a dedicated breast unit. ${ }^{9}$ 


\section{Diagnostic work-up and follow-up of recalled women}

A total of 15 regional and university hospitals were involved in the assessment of screen-positive women. The type of further diagnostic evaluation depended upon the workup protocols of the various hospitals and could include breast tomosynthesis, (3D) breast ultrasonography, magnetic resonance imaging, ultrasound guided fine-needle aspiration biopsy (FNAB) or core needle biopsy (CNB), stereotactic core needle biopsy (SCNB), or surgical excision biopsy. BI-RADS 0 lesions received additional imaging and biopsy if necessary. BI-RADS 4 and 5 lesions were routinely biopsied, whereas BI-RADS 3 lesions were either biopsied or followed-up. Data on mammographic breast density and family history of breast cancer were extracted from the radiological and surgical records, respectively. The follow-up period of all recalled women was two years (until the next biennial screen).

\section{Statistical analysis}

Descriptive statistics were performed using Statistical Package for Social Science 17.0 (SPSS Inc. Chicago, IL). A Fisher's exact test or chi-squared test was used to test differences in biopsy rates and workup procedures at non-blinded and blinded double reading.

\section{Results}

\section{Overall workup procedures after referral}

A total of 1235/42 996 (2.9\%) and 1474/ 44491 (3.3\%) women had been recalled, respectively at non-blinded and blinded double reading. Baseline characteristics for screened and recalled women were comparable for the two reading strategies (Table 4.2.1). At both reading strategies, workup consisted of additional breast imaging procedures only in about half of referrals, whereas the other half underwent a combination of additional breast imaging plus biopsy $(P=0.164$, Table 4.2 .2$)$. The vast majority of biopsy procedures in both groups consisted of either CNB or SCNB, whereas less than $3 \%$ of recalled women underwent FNAB and surgical biopsy was hardly used for diagnostic purposes. The distribution of various combinations of assessment at workup was also comparable, but the ultrasound guided CNB rate and SCNB rate per 1000 screens were lower at non-blinded than at blinded double reading (respectively 6.0 versus $7.5, P=0.008$ and 6.6 versus $8.1, P=0.009$ ). 
Table 4.2.1 Baseline characteristics of women screened and recalled at non-blinded and blinded double reading

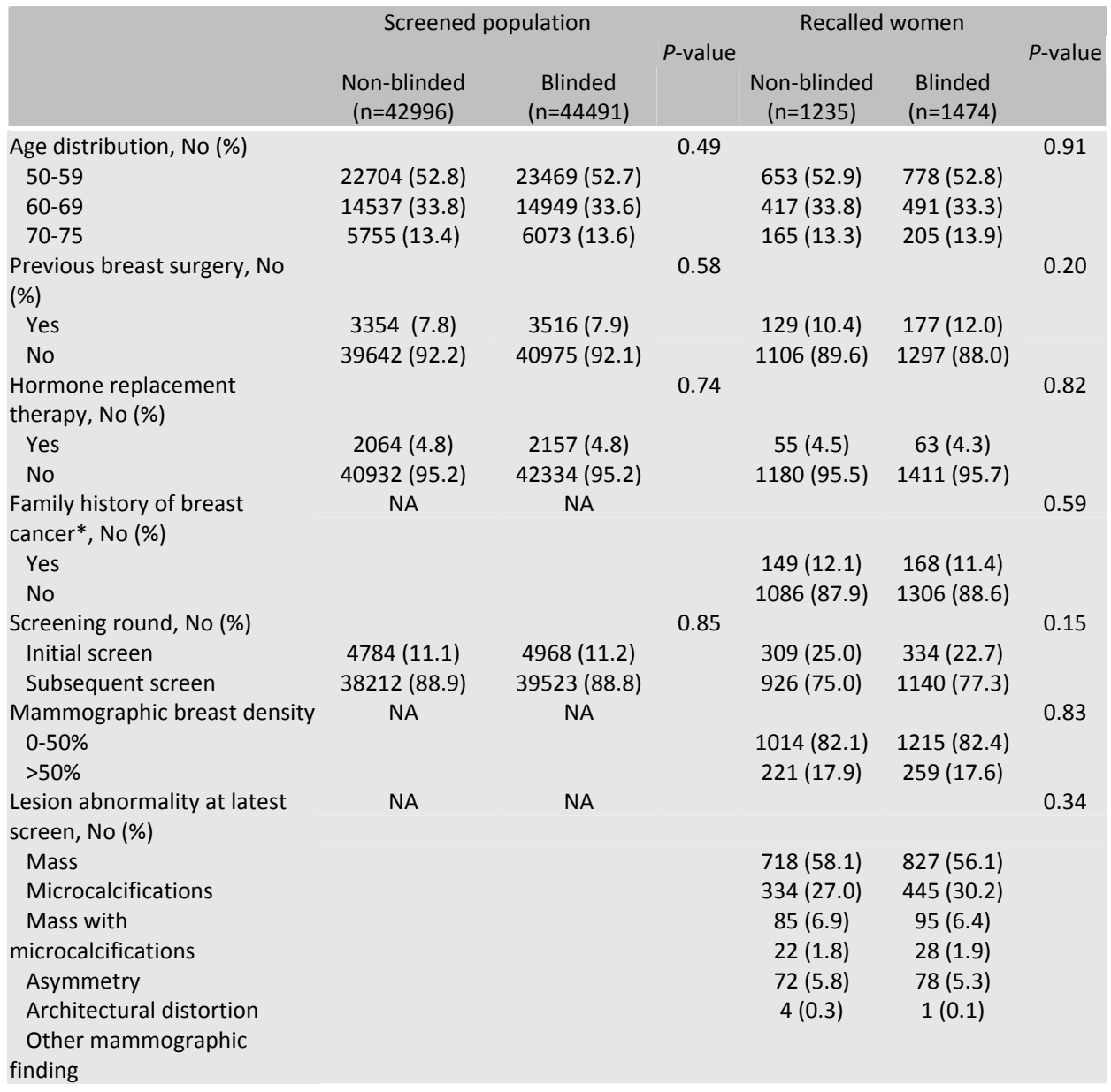

NA not available/not applicable. *At least one first-degree relative with a diagnosis of breast cancer before the age of 50 years or at least two second-degree relatives with breast cancer

\section{Workup up in false positive referrals}

A total of 951 (77.0\% of referrals) and 1149 (80.0\% of referrals) women experienced a false positive referral, respectively at non-blinded and blinded double reading (Table 4.2.3). The distribution of type of assessment differed between both groups, with a larger proportion of women evaluated by additional imaging only at non-blinded double reading $(65.0 \%$ versus $60.7 \%, P=0.046)$ and a larger proportion of CNB procedures at blinded double reading (9.9\% versus $6.5 \%, P=0.005)$. Almost all diagnostic surgical biopsies following referral had been performed in false positive cases, but the false positive surgical biopsy rate was still very low at both reading strategies (0.37 and 0.34 per 1000 screens at non-blinded and at blinded double 
reading, respectively). The CNB rate $(P<0.001)$ and SCNB rate $(P=0.013)$ per 1000 screens were higher at blinded than at non-blinded reading.

Table 4.2.2 Non-blinded versus blinded double reading at screening mammography: assessment following recall

\begin{tabular}{|c|c|c|c|c|c|c|c|c|}
\hline \multirow{3}{*}{$\begin{array}{l}\text { Assesment } \\
\text { recall }\end{array}$} & \multicolumn{5}{|c|}{ Proportion } & \multicolumn{3}{|c|}{ Rate (per 1000 screened women) } \\
\hline & \multicolumn{2}{|c|}{$\begin{array}{c}\text { Non-blinded } \\
(n=1235)\end{array}$} & \multicolumn{2}{|c|}{$\begin{array}{l}\text { Blinded } \\
(n=1474)\end{array}$} & \multirow[t]{2}{*}{$P$-value } & \multirow[t]{2}{*}{$\begin{array}{c}\text { Non-blinded } \\
(n=42996)\end{array}$} & \multirow[t]{2}{*}{$\begin{array}{c}\text { Blinded } \\
(n=44491)\end{array}$} & \multirow[t]{2}{*}{$P$-value } \\
\hline & $n$ & $\%$ & $n$ & $\%$ & & & & \\
\hline No further assessment & 3 & 0.2 & 0 & 0 & - & 0.1 & 0 & - \\
\hline Al & 618 & 50.0 & 698 & 47.4 & 0.164 & 14.4 & 15.7 & 0.110 \\
\hline$A l+F N A B$ & 29 & 2.3 & 36 & 2.4 & 0.873 & 0.7 & 0.8 & 0.465 \\
\hline $\mathrm{Al}+\mathrm{CNB}$ & 259 & 21.0 & 333 & 22.6 & 0.310 & 6.0 & 7.5 & 0.008 \\
\hline $\mathrm{Al}+\mathrm{SCNB}$ & 285 & 23.1 & 362 & 24.6 & 0.368 & 6.6 & 8.1 & 0.009 \\
\hline $\mathrm{Al}+\mathrm{FNAC}+\mathrm{CNB} / \mathrm{SCNB}$ & 16 & 1.3 & 18 & 1.2 & 0.862 & 0.4 & 0.4 & 0.808 \\
\hline $\mathrm{Al}+\mathrm{CNB} / \mathrm{FNAB} / \mathrm{SCNB}+\mathrm{SB}$ & 16 & 1.3 & 14 & 0.9 & 0.392 & 0.4 & 0.3 & 0.646 \\
\hline $\mathrm{Al}+\mathrm{CNB}+\mathrm{SCNB}$ & 8 & 0.6 & 12 & 0.8 & 0.614 & 0.2 & 0.3 & 0.413 \\
\hline $\mathrm{Al}+\mathrm{SB}$ & 1 & 0.1 & 1 & 0.1 & - & 0 & 0 & - \\
\hline
\end{tabular}

Al additional breast imaging; FNAB ultrasound guided fine-needle aspiration biopsy; CNB core needle biopsy; SCNB stereotactic core needle biopsy; SB surgical excision biopsy

Table 4.2.3 Non-blinded versus blinded double reading at screening mammography: assessment at false positive recall

\begin{tabular}{|c|c|c|c|c|c|c|c|c|}
\hline \multirow[t]{3}{*}{ Assesment following recall } & \multicolumn{5}{|c|}{ Proportion } & \multicolumn{3}{|c|}{ Rate (per 1000 screened women) } \\
\hline & \multicolumn{2}{|c|}{$\begin{array}{c}\text { Non-blinded } \\
(n=951)\end{array}$} & \multicolumn{2}{|c|}{$\begin{array}{l}\text { Blinded } \\
(n=1149)\end{array}$} & \multirow[t]{2}{*}{$P$-value } & \multirow[t]{2}{*}{$\begin{array}{c}\text { Non-blinded } \\
(n=42996)\end{array}$} & \multirow[t]{2}{*}{$\begin{array}{c}\text { Blinded } \\
(n=44491)\end{array}$} & \multirow[t]{2}{*}{$P$-value } \\
\hline & $\mathrm{n}$ & $\%$ & $\mathrm{n}$ & $\%$ & & & & \\
\hline No further assessment & 3 & 0.3 & 0 & 0 & - & 0.1 & 0 & - \\
\hline $\mathrm{Al}$ & 618 & 65.0 & 698 & 60.7 & 0.046 & 14.4 & 15.7 & 0.110 \\
\hline $\mathrm{Al}+\mathrm{FNAB}$ & 28 & 2.9 & 35 & 3.0 & 0.892 & 0.7 & 0.8 & 0.455 \\
\hline $\mathrm{Al}+\mathrm{CNB}$ & 62 & 6.5 & 114 & 9.9 & 0.005 & 1.4 & 2.6 & $<0.001$ \\
\hline $\mathrm{Al}+\mathrm{SCNB}$ & 200 & 21.0 & 261 & 22.7 & 0.353 & 4.7 & 5.9 & 0.013 \\
\hline $\mathrm{Al}+\mathrm{FNAC}+\mathrm{CNB} / \mathrm{SCNB}$ & 16 & 1.7 & 15 & 1.3 & 0.476 & 0.4 & 0.3 & 0.783 \\
\hline $\mathrm{Al}+\mathrm{CNB} / \mathrm{FNAB} / \mathrm{SCNB}+\mathrm{SB}$ & 16 & 1.7 & 14 & 1.2 & 0.372 & 0.4 & 0.3 & 0.646 \\
\hline $\mathrm{Al}+\mathrm{CNB}+\mathrm{SCNB}$ & 8 & 0.8 & 11 & 1.0 & 0.780 & 0.2 & 0.2 & 0.539 \\
\hline $\mathrm{Al}+\mathrm{SB}$ & 0 & 0 & 1 & 0.1 & - & 0 & 0 & - \\
\hline
\end{tabular}

Al additional breast imaging; FNAB ultrasound guided fine-needle aspiration biopsy; CNB core needle biopsy; SCNB stereotactic core needle biopsy; SB surgical excision biopsy

\section{Overall biopsy rates and positive predictive value of biopsy}

The two reading strategies showed comparable positive predictive value of biopsy (46.3\% versus $41.9 \%$ at respectively non-blinded and blinded double reading, $P=0.103$, Table 4.2.4). Both the overall biopsy rate and benign biopsy rate per 1000 screens were higher at blinded double reading (respectively 17.4 versus $14.3(P<0.001)$ and 10.1 versus $7.7, P<0.001$ ), as well as the proportion of women who underwent biopsy as part of a false positive referral $(39.3 \%$ versus $34.7 \%, P=0.032)$. 
Table 4.2.4 Biopsy rates and positive predictive values of biopsy at blinded and non-blinded double reading of screening mammograms

\begin{tabular}{lllc} 
& Non-blinded & \multicolumn{1}{c}{ Blinded } & $P$-value \\
\hline Assessment at recall & & & 0.129 \\
$\quad$ Biopsy, \% & $49.7(614 / 1235)$ & $52.6(776 / 1474)$ & \\
No biopsy, \% & $50.3(621 / 1235)$ & $47.4(698 / 1474)$ & \\
Biopsy rate per 1000 screens & $14.3(614 / 42996)$ & $17.4(776 / 44491)$ & $<0.001$ \\
PPV of biopsy, \% & $46.3(284 / 614)$ & $41.9(325 / 776)$ & 0.103 \\
False positive recall & & & 0.032 \\
$\quad$ Biopsy, \% & $34.7(330 / 951)$ & $39.3(451 / 1149)$ & \\
$\quad$ No biopsy, \% & $65.3(621 / 951)$ & $60.7(698 / 1149)$ & $<0.001$ \\
Benign biopsy rate per 1000 screens & $7.7(330 / 42996)$ & $10.1(451 / 44491)$ & $<$
\end{tabular}

\section{Discussion}

We found that blinded double reading resulted in significantly higher CNB and SCNB rates than non-blinded double reading, as well as a significantly higher benign biopsy rate. A larger proportion of women at benign work-up were evaluated merely by additional imaging at non-blinded double reading, whereas a larger fraction of referrals underwent CNB procedures at blinded double reading. The PPV of biopsy was comparable for both reading strategies.

The current study included screens during the first round of digital screening in the Netherlands. We found a biopsy rate of 14.3 per 1000 screens after non-blinded double reading and 17.4 per 1000 screens after blinded double reading. To our knowledge, data of biopsy rates after blinded double reading have not been reported previously. The biopsy rates we observed after non-blinded double reading are comparable to those of a Norwegian (61/4009) and Irish (470/35204) study during the first round of digital mammography. ${ }^{10,11}$

In a Dutch study, increased detection of lesions presenting as microcalcifications during the transition from screen-film to digital screening mammography resulted in an almost fourfold increase in SCNB per thousand screened women. ${ }^{8}$ The further intensified use of SCNB at blinded double reading may have a great impact on daily practice of regional breast clinics as it is much more time consuming than conventional breast imaging or ultrasound guided biopsy.

Among women with benign workup, a larger fraction was evaluated simply by additional imaging at non-blinded double reading, whereas a larger fraction at blinded double reading also underwent CNB procedures. The higher referral rate at blinded double reading results in higher costs of the diagnostic procedures in the hospital, which will rise even further as a larger fraction of referrals is evaluated by CNB in addition to breast imaging at blinded double reading.

The higher biopsy rates at blinded double readings was accompanied by an increased cancer detection rate, which resulted in a comparable PPV of biopsy of more 
than $40 \%$ at both reading strategies. This PPV is within the range of those found in other European programs and higher than those observed in the US. ${ }^{10,12,13}$

It remains a question of debate whether false-positive referrals, which received additional imaging or biopsy, have uniformly decreased odds of attending subsequent routine screening rounds. ${ }^{14-16}$ Many of the women with false positive screening results experience distress, ${ }^{17}$ which may last for up to three years after a false positive screen and is related to the invasiveness of the assessment. ${ }^{18}$ It is therefore important to keep the number of benign biopsies as low as possible.

Our study has certain limitations. The study population consisted of women who attended the screening program during the transition from screen-film to digital screening mammography. Therefore, the majority of subsequent screens were compared with previously obtained screen-film mammograms. Data on screening outcome after the transition to digital screening mammography are sparse and there are no studies available that compare the use of diagnostic workup modalities during and after the transition. All screening examinations in our study comprised two-view mammography of both breasts, including a medio-lateral-oblique view and a craniocaudal view. Recent studies have shown that the addition of breast tomosynthesis to digital mammography improves the cancer detection rate in mammography screening and may reduce false positive referrals. ${ }^{19}$ Breast tomosynthesis, however, has not yet been implemented in the Dutch mammography screening program.

In summary, we conclude that blinded double reading resulted in significantly higher overall and benign biopsy rate than non-blinded double reading. Although an increased screening sensitivity at blinded double reading has been reported, the negative side effect of higher biopsy rates should be taken into account when adopting this reading strategy. 


\section{References}

1 Kalager M, Zelen M, Langmark F, Adami HO. Effect of screening mammography on breast-cancer mortality in Norway. N Engl J Med 2010;363:1203-1210.

2 Mandelblatt J, van Ravesteyn N, Schechter C et al. Which strategies reduce breast cancer mortality most? Collaborative modeling of optimal screening, treatment, and obesity prevention. Cancer 2013;119:2541-2548.

3 van Schoor G, Moss SM, Otten JD et al. Increasingly strong reduction in breast cancer mortality due to screening. Br J Cancer 2011;104:910-914.

4 Duijm LE, Louwman MW, Groenewoud JH, van de Poll-Franse LV, Fracheboud J, Coebergh JW. Interobserver variability in mammography screening and effect of type and number of readers on screening outcome. Br J Cancer 2009;100:901-907.

5 Gur D, Sumkin JH, Hardesty LA et al. Recall and detection rates in screening mammography. Cancer 2004;100:1590-1594.

6 Klompenhouwer EG, Voogd AC, den Heeten GJ et al. Blinded double reading yields a higher programme sensitivity than non-blinded double reading at digital screening mammography: A prospected population based study in the south of The Netherlands. Eur J Cancer 2015;51:391-399.

7 Fracheboud J, de Koning HJ, Boer R et al. Nationwide breast cancer screening programme fully implemented in The Netherlands. Breast 2001;10:6-11.

8 Nederend J, Duijm LE, Louwman MW, Groenewoud JH, Donkers-van Rossum AB, Voogd AC. Impact of transition from analog screening mammography to digital screening mammography on screening outcome in The Netherlands: a population-based study. Ann Oncol 2012;23:3098-3103.

9 D'Orsi CJ, Mendelson EB, Ikeda DM, et al. Breast Imaging Reporting and Data System: ACR BI-RADS Breast Imaging Atlas. American College of Radiology, Reston, VA. 2003.

10 Skaane $P$, Hofvind S, Skjennald A. Randomized trial of screen-film versus full-field digital mammography with soft-copy reading in population-based screening program: follow-up and final results of Oslo II study. Radiology 2007;244:708-717.

11 Hambly NM, McNicholas MM, Phelan N, Hargaden GC, O'Doherty A, Flanagan FL. Comparison of digital mammography and screen-film mammography in breast cancer screening: a review in the Irish breast screening program. AJR Am J Roentgenol 2009;193:1010-1018.

12 Glynn CG, Farria DM, Monsees BS, Salcman JT, Wiele KN, Hildebolt CF. Effect of transition to digital mammography on clinical outcomes. Radiology 2011;260:664-670.

13 Vinnicombe S, Pinto Pereira SM, McCormack VA, Shiel S, Perry N, Silva IMD. Full-Field Digital versus Screen-Film Mammography: Comparison within the UK Breast Screening Program and Systematic Review of Published Data. Radiology 2009;251:347-358.

14 Fitzpatrick P, Fleming P, O'Neill S, Kiernan D, Mooney T. False-positive mammographic screening: factors influencing re-attendance over a decade of screening. J Med Screen 2011;18:30-33.

15 Maxwell AJ, Beattie C, Lavelle J et al. The effect of false positive breast screening examinations on subsequent attendance: retrospective cohort study. J Med Screen 2013;20:91-98.

16 Seigneurin A, Exbrayat C, Labarere J, Delafosse P, Poncet F, Colonna M. Association of diagnostic workup with subsequent attendance in a breast cancer screening program for false-positive cases. Breast Cancer Res Treat 2011;127:221-228.

17 van der Steeg AF, Keyzer-Dekker CM, De Vries J, Roukema JA. Effect of abnormal screening mammogram on quality of life. Br J Surg 2011;98:537-542.

18 Bond M, Pavey T, Welch K et al. Psychological consequences of false-positive screening mammograms in the UK. Evid Based Med 2013;18:54-61.

19 Friedewald SM, Rafferty EA, Conant EF. Breast cancer screening with tomosynthesis and digital mammography-reply. JAMA 2014;312:1695-1696. 


\section{Chapter 4.3}

Discrepant screening mammography assessments at blinded and non-blinded double reading: impact of arbitration by a third reader on screening outcome

Klompenhouwer EG, Voogd AC, den Heeten GJ, Strobbe LA, Tjan-Heijnen VCG, Broeders MJM, Duijm LEM

Eur Radiol. 2015 Apr 18. (epub ahead of print) 


\section{Abstract}

Purpose

To determine the value of adding a third reader for arbitration of discrepant screening mammography assessments.

\section{Methods}

We included a consecutive series of 84,927 digital screening mammograms, double read in a blinded or non-blinded fashion. Arbitration was retrospectively performed by a third screening radiologist. Two years follow-up was performed.

\section{Results}

Discrepant readings comprised $57.2 \%$ (830/1452) and 29.1\% (346/1188) of the recalls at blinded and non-blinded double readings, respectively. At blinded double reading, arbitration would have decreased the recall rate $(3.4 \%$ to $2.2 \%, P<0.001)$ and program sensitivity $(83.2 \%$ to $76.0 \%$, $P=0.013$ ), would not have influenced the cancer detection rate (CDR, 7.5 to 6.8 per 1000 screens, $P=0.258$ ) and would have increased the positive predictive value of recall (PPV, $22.3 \%$ to $31.2 \%$, $P<0.001)$. At non-blinded double reading, arbitration would have decreased the recall rate $(2.8 \%$ to $2.3 \%, P<0.001)$ and increased the PPV $(23.2 \%$ to $27.5 \%, P=0.021)$, but would not have affected the CDR ( 6.6 to 6.3 per 1,000 screens, $P=0.604$ ) and program sensitivity ( 76.0 to $72.7 \%, P=0.308$ ).

\section{Conclusion}

We conclude that arbitration of discrepant screening mammography assessments is a good tool to improve recall rate and PPV, but is not desirable as it reduces the program sensitivity at blinded double reading. 


\section{Introduction}

Reading strategies of organized screening programs can influence the main performance parameters in a substantial way and for that reason can influence what often is considered the possible harms and benefits of screening. Analysis and comparisons of these strategies can help decision making and may be useful for screening programs in general.

Reading of screening mammograms can be performed in several ways, e.g. by single radiologist reading, single reading with computer aided detection (CAD) or double reading. Double reading by two specialized breast screening radiologists is considered to be the standard of reference ${ }^{1}$ and this reading strategy significantly increases the cancer detection rate when compared to single reading. ${ }^{2-6}$

Double reading can be performed either in a blinded fashion $\left(2^{\text {nd }}\right.$ reader is not informed about the $1^{\text {st }}$ reader's decision) or non-blinded fashion $\left(2^{\text {nd }}\right.$ reader is informed about the $1^{\text {st }}$ reader's decision). In the Netherlands, mammograms were usually read in a non-blinded fashion in the era of screen-film mammography. With the introduction of full-field digital mammography (FFDM) it became technically feasible to perform blinded double reading as an alternative to non-blinded double reading. We recently showed that blinded double reading at FFDM resulted in a significantly better program sensitivity of screening mammography compared to non-blinded double reading, at the expense of a higher overall recall rate and false positive recall rate. ${ }^{7}$ In this study, women with a discrepant reading between two screening radiologists were always recalled for further analysis. Alternatively, the two radiologists may try to reach consensus in case of a discrepant reading, or arbitration by a third radiologist may be considered. In case of a discrepant reading, one of these alternative reading strategies may lower the recall rate and amount of false positive recalls, but possibly at the expense of a lower detection rate.

A few studies have evaluated the effect of consensus meetings ${ }^{8}$ and arbitration ${ }^{9-11}$ on screening outcome at screen film mammography (SFM) at independent or nonblinded double reading. These studies showed that arbitration and consensus meetings resulted in reduced recall rates, at the expense of a small decrease in cancer detection rate.

The purpose of the current study is to determine the potential effect of arbitration by a third reader of discrepant readings in the specific setting of blinded and nonblinded double reading at digital screening mammography and to determine the mammographic abnormalities and tumor characteristics of cancers missed after arbitration. 


\section{Methods}

\section{Study population}

We included a consecutive series of 84,927 full-field digital screening mammograms performed between July 1, 2009 and July 1, 2011. The screens were obtained at three specialized screening units in a southern biennial screening mammography region of the Netherlands (BOZ, Bevolkings Onderzoek Zuid).

Women participating in the nationwide Dutch screening program are routinely asked to give permission for the use of their data for scientific purposes. Two women screened at our units did not give this permission and they were excluded. This study was performed within the national permit for breast cancer screening, which is issued by the Ministry of Health, Welfare and Sports after advice of the Dutch Health Council. This study did not require a special permit according to the Dutch Law on Populationbased screening as both blinded and non-blinded double reading were considered "standard of care" at the time of the study.

\section{Screening procedure and recall}

Details of the nationwide breast cancer screening program have been described previously., ${ }^{9,12}$ In brief, this program provides free biennial screening mammography for women aged 50-75 years and is organized by a system of personal invitations with a program compliance rate of $80 \%$.

Mammograms were acquired with a Lorad Selenia FFDM system (Hologic Inc, Danbury, CT), with a $70 \mu \mathrm{m}$ pixel size and a $232 \times 286 \mathrm{~mm}$ field of view. The mammographic examinations were obtained by specialized screening mammography technologists and double read in changing duo's of a team of 12 certified screening radiologists. Each radiologist evaluated at least 6,000 screening mammograms yearly. Prior screening mammograms were always available for comparison the time of a subsequent screening round. To facilitate comparison of subsequent FFDM screens with prior SFM screens, the most recent screen-film mammograms were digitized by fusion equipments designed for mammography (DigitalNow;R2/Hologic).

Screening mammograms were double read in either a blinded $\left(2^{\text {nd }}\right.$ reader was not informed about the $1^{\text {st }}$ reader's decision) or non-blinded $\left(2^{\text {nd }}\right.$ reader was informed about the $1^{\text {st }}$ reader's decision). This reading strategy was alternated on a monthly basis for the purpose of the current study. During the study period, women with discrepant readings between the two screening radiologists, at blinded and non-blinded double reading, were always recalled for further analysis. Fur the purpose of the study, each discrepant reading was then randomly assigned to a third screening radiologist, who retrospectively decided whether or not he/she would have recalled the woman. This third reader was blinded to the screening outcome.

Each screen was classified according to the Breast Imaging Reporting and Data System (BI-RADS). ${ }^{13}$ Women with a screening BI-RADS 0, 4 or 5 were recalled to a 
dedicated breast unit of a hospital. At screening, mammographic abnormalities were classified according to the following categories: suspicious mass, suspicious microcalcifications, suspicious mass in combination with microcalcifications, architectural distortion, asymmetry or other abnormality.

\section{Diagnostic work-up and follow-up of recalled women}

A total of 15 regional and university hospitals were involved in the assessment of screen-positive women. After physical examination by the surgeon, additional mammographic views were obtained if necessary. At diagnostic workup, radiologists again classified the radiologic findings according to the BI-RADS. ${ }^{13}$ Dependent on the findings at physical examination and the diagnostic workup protocols at the various hospitals, further diagnostic evaluation could include breast ultrasonography, magnetic resonance mammography, percutaneous fine-needle aspiration biopsy (FNAB), core needle biopsy (CNB) or open excision biopsy. BI-RADS 4 and 5 lesions were routinely biopsied, whereas BI-RADS 3 lesions were either biopsied or followed-up.

During a follow-up period of at least two years (until the next biennial screening round), we collected data on diagnostic imaging procedures, clinical data, biopsy specimen and surgical procedures of all recalled women. Breast cancers were divided into ductal carcinoma in situ (DCIS) and invasive cancer. Lobular carcinoma in situ was left out of consideration. Data on TNM (tumor-node-metastases) classification [14] were collected. Sentinel nodes were classified negative if they harbored isolated tumor cells or sub-micrometastases $\left(<0.2 \mathrm{~mm}, \mathrm{NO}_{(\text {itc })}\right)$ and were considered positive if they contained micrometastases $\left(0.2-2 \mathrm{~mm}, \mathrm{N1}{ }_{(\mathrm{mi})}\right)$ or macrometastases (>2 mm, N1-3).

\section{Detection of interval cancers}

Interval cancers are breast cancers diagnosed after a negative screening mammography (i.e., screening without a recommendation for referral), and before the next biennial screening examination. Calculation of the program sensitivity of the screening strategies requires identification of all interval cancers.

We traced interval cancers, by using all available information in addition to the usual follow-up procedure, including:

- All radiotherapy reports that were received from the regional radiotherapy institutes concerning women who underwent radiotherapy for breast malignancy and who had participated in the screening program;

- Inquiry about pathology specimens at the various regional pathology laboratories, some months after any hospital had requested the screening mammograms of a woman who had not been referred for further analysis;

- The pathology records that were obtained if a woman cancelled a call for subsequent screening because breast cancer had been diagnosed after a previous negative screen; 
- The occasional reports on interval cancers provided by general practitioners or medical specialists to the screening centre.

\section{Statistical analysis}

The main outcome measures of the reading strategies were recall rate (recalls per 100 screens), cancer detection rate (CDR, screen detected cancers per 1000 screens), proportion of BI-RADS 0 among all recalls, positive predictive value (PPV, screen detected cancers per 100 recalls) and program sensitivity. Screening program sensitivity was calculated by determination of the proportion of screen-detected cancers out of all breast cancers (screen-detected and interval cancers) diagnosed during the 2-year screening interval. For the purpose of this study, cancers not recalled after arbitration by a third reader were calculated as interval cancers.

The results were analyzed using an independent-sample t-test. A one-sample t-test was used to determine the $95 \%$ confidence interval $(95 \% \mathrm{Cl})$. A Chi square and Fisher exact test were used to test differences in tumor and mammographic characteristics of the reading strategies and to test differences in surgical treatment. A p-value of less than 0.05 was considered to indicate statistical significance.

Statistics were performed using Statistical Package for Social Science 17.0 (SPSS Inc. Chicago, IL) and SAS ${ }^{\circledR} 9$.

\section{Results}

\section{Overall screening results}

Respectively 43,184 and 41,743 screens had been double read, either in a blinded or non-blinded fashion. The mean age of both study groups was similar (both 59 years, $95 \% \mathrm{Cl} 59-60)$. The screening results of blinded and non-blinded reading are published elsewhere. ${ }^{7}$ The recall rate $(3.3 \%$ versus $2.8 \%, P<0.001)$, as well as the proportion of discrepant readings among recalled women (57.2\% versus $29.1 \%, P<0.001$ ), was significantly higher at blinded double reading than at non-blinded reading (Figure 4.3.1).

\section{Blinded double reading}

Of the 830 discrepant readings at blinded double reading, 323 (38.9\%) would have been recalled after arbitration, of which 65 (20.1\%) proved to be malignant. Of the 507 screens, not recalled after arbitration, $28(5.5 \%)$ proved to be malignant, and calculated as interval cancers for the purpose of this study (Figure 4.3.1). 


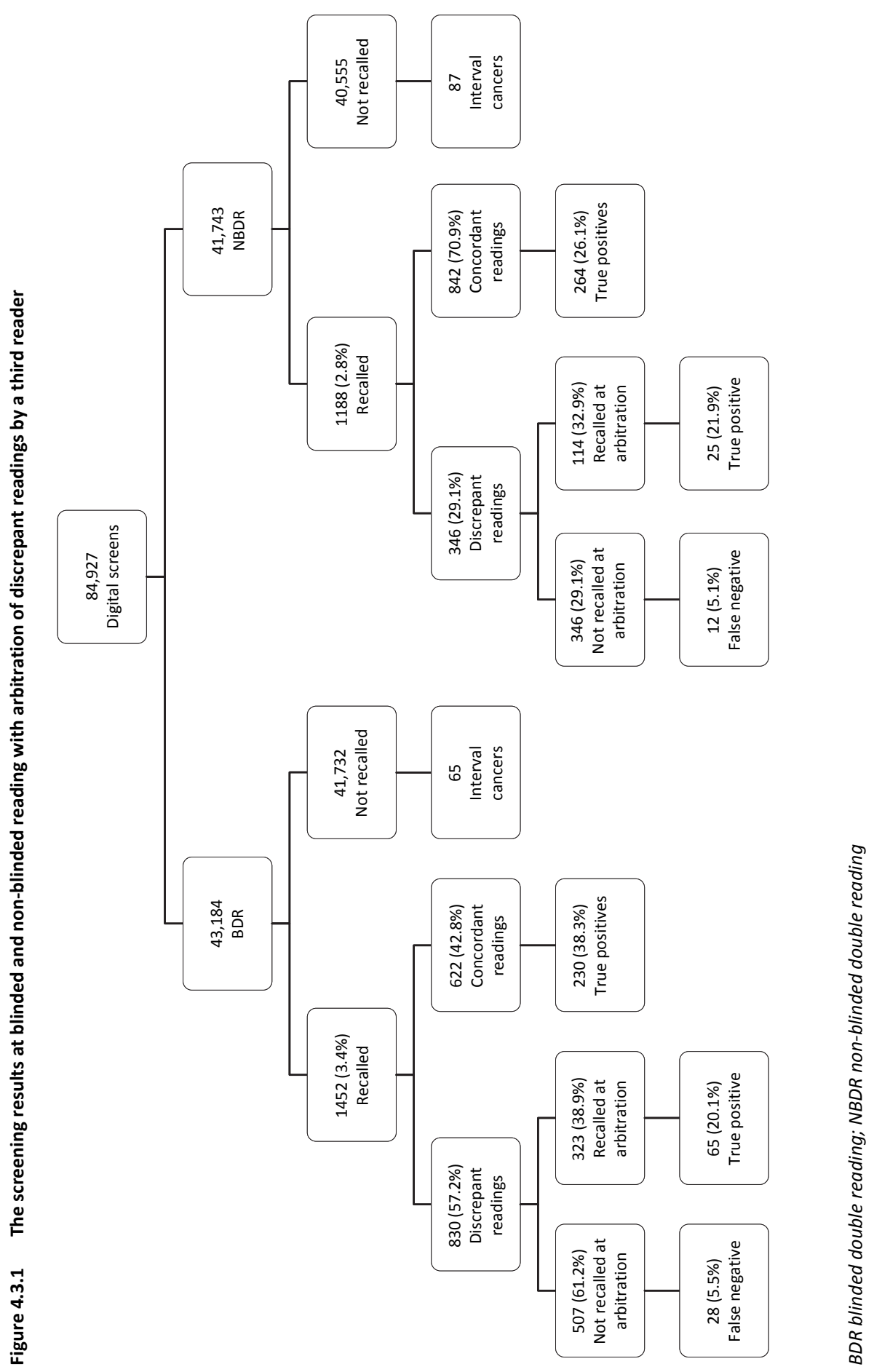


The screening results at blinded double reading without and with arbitration of discrepant readings by a third reader are shown in Table 4.3.1. At blinded double reading, arbitration of discrepant readings would have decreased the recall rate from $3.4 \%$ to $2.2 \%(P<0.001)$ and the proportion of BI-RADS 0 among recalls from $32.5 \%$ to $23.0 \%(P<0.001)$ and would have increased the PPV from $22.3 \%$ to $31.2 \%(P<0.001)$. Arbitration would also have resulted in a significantly lower program sensitivity (from $83.2 \%$ to $76.0 \%, P=0.013$ ), but not influenced the CDR (from 7.5 to 6.8 per 1000 screens, $P=0.258)$.

Table 4.3.1 Screening results of 43,184 blinded double readings without and with arbitration of discrepant readings by a third reader

\begin{tabular}{|c|c|c|c|}
\hline & $\begin{array}{c}\text { Recall of all } \\
\text { discrepant readings }\end{array}$ & $\begin{array}{c}\text { Recall after } \\
\text { arbitration of } \\
\text { discrepant readings }\end{array}$ & $P$-value \\
\hline Recalls, No $(95 \% \mathrm{Cl})$ & 1452 & 945 & \\
\hline Recall rate, $\%(95 \% \mathrm{CI})$ & $3.4(3.2-3.5)$ & $2.2(2.1-2.3)$ & $<0.001$ \\
\hline Proportion of BI-RADS 0 among recalls*, $\%(95 \% \mathrm{CI})$ & $32.5(30.1-34.9)$ & $23.0(20.3-25.7)$ & $<0.001$ \\
\hline Screen detected cancers, No & 323 & 295 & \\
\hline Interval cancers, No & 65 & $93 * *$ & \\
\hline Cancer detection rate, $\%$ ( $95 \% \mathrm{Cl})$ & $7.5(6.7-8.3)$ & $6.8(6.1-7.6)$ & 0.258 \\
\hline Positive predictive value of recall, $\%(95 \% \mathrm{Cl})$ & $22.3(20.1-24.4)$ & $31.2(28.3-34.2)$ & $<0.001$ \\
\hline Program sensitivity, $\%(95 \% \mathrm{Cl})$ & $83.2(79.5-87.0)$ & $76.0(71.8-80.3)$ & 0.013 \\
\hline
\end{tabular}

* Highest BI-RADS classification among the two readers; ** 65 true interval cancers and 28 cancers not recalled after arbitration

\section{Non-blinded double reading}

Of the 346 discrepant readings at non-blinded double reading, 114 (32.9\%) would have been recalled after arbitration, of which 25 (21.9\%) proved to be malignant. Of the 232 screens not recalled after arbitration, $12(5.1 \%)$ proved to be malignant, and calculated as interval cancers for the purpose of this study (Figure 4.3.1).

The screening results at blinded double reading, without and with arbitration of discrepant readings by a third reader, are shown in Table 4.3.2. At non-blinded double reading, arbitration of discrepant readings would have decreased the recall rate from $2.8 \%$ to $2.3 \%(P<0.001)$ and the proportion of BI-RADS 0 among recalls from $32.0 \%$ to $26.7 \%(P=0.007)$ and would have increased the PPV of recall from $23.2 \%$ to $27.5 \%$ $(P=0.021$ ). Arbitration did not influence the CDR (from 6.6 to 6.3 per 1,000 screens, $P=0.604$ ) and program sensitivity (from 76.0 to $72.7 \%, P=0.308$ ). 
Table 4.3.2 Screening results of 41,743 non-blinded double readings without and with arbitration of discrepant readings by a third reader

\begin{tabular}{|c|c|c|c|}
\hline & $\begin{array}{c}\text { Recall of all } \\
\text { discrepant readings }\end{array}$ & $\begin{array}{c}\text { Recall after } \\
\text { arbitration of } \\
\text { discrepant readings }\end{array}$ & P-value \\
\hline Recalls, No (95\% Cl) & 1188 & 956 & \\
\hline Recall rate, $\%(95 \% \mathrm{Cl})$ & $2.8(2.7-3.0)$ & $2.3(2.1-2.4)$ & $<0.001$ \\
\hline Proportion of BI-RADS 0 among recalls*, $\%(95 \% \mathrm{CI})$ & $32.0(29.3-34.6)$ & $26.7(23.9-29.5)$ & 0.007 \\
\hline Screen detected cancers & 275 & 263 & \\
\hline Interval cancers & 87 & $99 * *$ & \\
\hline Cancer detection rate, \%о $(95 \% \mathrm{Cl})$ & $6.6(5.8-7.4)$ & $6.3(5.5-7.1)$ & 0.604 \\
\hline Positive predictive value of recall, $\%(95 \% \mathrm{Cl})$ & $23.2(20.8-25.6)$ & $27.5(24.7-30.3)$ & 0.021 \\
\hline Program sensitivity, \% $(95 \% \mathrm{Cl})$ & $76.0(71.5-80.4)$ & $72.7(68.0-77.3)$ & 0.308 \\
\hline
\end{tabular}

* Highest BI-RADS classification among the two readers; ** 87 true interval cancers and 12 cancers not recalled after arbitration

\section{Mammographic abnormalities, tumor characteristics and type of breast surgery of screen detected cancers and cancers missed after arbitration}

The mammographic abnormalities of screen-detected cancers and cancers that would have been missed after arbitration of discrepant readings by a third reader were largely comparable $(P=0.061)$. A suspicious mass was the most frequently encountered reason of recall (about $50 \%$ of recalls), followed by suspicious microcalcifications (Table 4.3.3).

Cancers missed after arbitration of discrepant readings had more frequently been recalled as a BI-RADS 0 lesion, whereas screen detected cancers (SDCs) had more frequently been recalled as BI-RADS 5 lesions $(P<0.001)$. Cancers in dense breasts were not more likely to be missed at arbitration ( $P=0.712$, Table 4.3.3).

We observed no differences in the proportion of DCIS $(24.2 \%$ versus $32.5 \%$, $P=0.240)$, the proportion of smaller (T1a-c) tumors $(80.6 \%$ versus $81.5 \%, P=0.936)$, lymph node involvement $(20.3 \%$ versus $11.1 \%, P=0.323)$ and the proportion of advanced tumors among SDCs and cancers missed at arbitration. However, among DCIS, missed tumors at arbitration more often had a low grade than screen detected DCIS (28.9\% versus $69.2 \%, P=0.009)$ and invasive cancers with axillary lymph node metastasis were less often seen among cancers missed at arbitration (20.3\% versus $11.1 \%, P<0.001$,Table 4.3.3).

The histologic subtypes and tumor grading of invasive cancers were comparable for both SDCs and cancers missed at arbitration. The majority of invasive cancers, about $80 \%$, were of the ductal type, followed by invasive lobular cancer (10-15\%). Just over $50 \%$ of invasive cancers were Nottingham grade I, both among SDCs and cancers missed at arbitration $(P=0.794)$. The proportions of estrogen, progesterone or Her2/Neu receptor positive invasive cancers were also comparable, as well as the proportion of triple negative invasive cancers. Finally, there was no difference regarding the type of surgery, i.e. breast conserving surgery or mastectomy, for SDCs and cancers missed at arbitration $(P=0.191$, Table 4.3 .3$)$. Over $80 \%$ of the screen- 
detected cancers and cancers missed at arbitration were treated by breast conserving surgery.

Table 4.3.3: Mammographic abnormalities, tumor characteristics and type of breast surgery of screen detected cancers and cancers missed at arbitration of discrepant readings by a third reader, at blinded and non-blinded double reading

\begin{tabular}{|c|c|c|c|}
\hline & $\begin{array}{c}\text { SDCS } \\
(n=558)\end{array}$ & $\begin{array}{l}\text { Cancers missed } \\
\text { at arbitration } \\
\quad(n=40)\end{array}$ & $P$-value \\
\hline Mammographic abnormality, $\mathrm{n}(\%)$ & & & 0.061 \\
\hline Mass & $292(52.3)$ & $19(47.5)$ & \\
\hline Microcalcifications & $161(28.9)$ & $14(35.0)$ & \\
\hline Mass with microcalcifications & $68(12.2)$ & $2(5.0)$ & \\
\hline Asymmetry & $1(0.2)$ & $1(2.5)$ & \\
\hline Architectural distortion & $36(6.5)$ & $4(10.0)$ & \\
\hline Breast density of screening mammogram, $\mathrm{n}(\%)$ & & & 0.712 \\
\hline$\leq 50 \%$ & 446 (79.9) & $31(77.5)$ & \\
\hline$>50 \%$ & $112(20.1)$ & $9(22.5)$ & \\
\hline BI-RADS at recall*, n (\%) & & & $<0.001$ \\
\hline 0 & $46(8.2)$ & $13(32.5)$ & \\
\hline 4 & 407 (72.9) & $27(67.5)$ & \\
\hline 5 & $105(18.8)$ & $0(0.0)$ & \\
\hline Type of screen detected cancer, n (\%) & & & 0.240 \\
\hline DCIS & $135(24.2)$ & $13(32.5)$ & \\
\hline Invasive cancers & $423(75.8)$ & $27(67.5)$ & \\
\hline DCIS & $\mathrm{N}=135$ & $\mathrm{~N}=13$ & \\
\hline Grade & & & 0.009 \\
\hline Low & 39 (28.9) & $9(69.2)$ & \\
\hline Intermediate & $46(34.1)$ & $3(23.1)$ & \\
\hline High & $50(37.0)$ & $1(7.7)$ & \\
\hline Invasive cancers & $\mathrm{N}=423$ & $\mathrm{~N}=27$ & \\
\hline Histology type & & & 0.467 \\
\hline Ductal & $341(80.6)$ & $21(77.8)$ & \\
\hline Lobular & $40(9.5)$ & $4(14.8)$ & \\
\hline Mixed ductal/lobular & $22(5.2)$ & $0(0.0)$ & \\
\hline Other & $20(4.7)$ & $2(7.4)$ & \\
\hline Size of invasive cancer & & & 0.936 \\
\hline T1a-c & $342(80.6)$ & $22(81.5)$ & \\
\hline $\mathrm{T} 2+$ & $81(19.4)$ & $5(18.5)$ & \\
\hline Lymph node status & & & 0.323 \\
\hline Positive & $86(20.3)$ & $3(11.1)$ & \\
\hline Negative & 337 (79.7) & $24(88.9)$ & \\
\hline Tumor stage** & & & 1.000 \\
\hline Advanced & $129(23.1)$ & $6(22.2)$ & \\
\hline Not-advanced & 429 (76.9) & $21(77.8)$ & \\
\hline Nottingham grade & & & 0.794 \\
\hline Nottingham I & $215(50.8)$ & $14(51.9)$ & \\
\hline Nottingham II & $172(40.7)$ & $10(37.0)$ & \\
\hline Nottingham III & $30(7.1)$ & $3(11.1)$ & \\
\hline Unknown & $6(1.4)$ & $0(0.0)$ & \\
\hline
\end{tabular}


Table 4.3.3 (continued)

\begin{tabular}{|c|c|c|c|}
\hline & $\begin{array}{c}\text { SDCs } \\
(n=558)\end{array}$ & $\begin{array}{l}\text { Cancers missed } \\
\text { at arbitration } \\
\quad(n=40)\end{array}$ & $P$-value \\
\hline Estrogen receptor & & & 0.832 \\
\hline Positive & $380(89.8)$ & $25(92.6)$ & \\
\hline Negative & $39(9.2)$ & $2(7.4)$ & \\
\hline Unknown & $4(1.0)$ & $0(0.0)$ & \\
\hline Progesteron receptor & & & 0.641 \\
\hline Positive & $296(70.0)$ & $21(77.8)$ & \\
\hline Negative & $123(29.0)$ & $6(12.2)$ & \\
\hline Unknown & $4(1.0)$ & $0(0.0)$ & \\
\hline Her2/Neu receptor & & & 0.796 \\
\hline Positive & $37(8.7)$ & $2(7.4)$ & \\
\hline Negative & $380(90.8)$ & $25(92.6)$ & \\
\hline Unknown & $6(1.4)$ & $0(0.0)$ & \\
\hline Triple- receptor negative & & & 0.823 \\
\hline Yes & $30(7.1)$ & $2(7.4)$ & \\
\hline No & $387(91.5)$ & $25(92.6)$ & \\
\hline Unknown & $6(1.4)$ & $0(0.0)$ & \\
\hline \multicolumn{4}{|l|}{ DCIS/Invasive cancers } \\
\hline Type of surgery & & & 0.191 \\
\hline Breast conserving & $455(81.5)$ & $34(85.0)$ & \\
\hline Mastectomy & $94(16.8)$ & $4(10.0)$ & \\
\hline No surgery & $7(1.3)$ & $2(5.0$ & \\
\hline Unknown & $2(0.4)$ & $0(0.0)$ & \\
\hline
\end{tabular}

\section{Discussion}

To our knowledge, this is the first study reporting on the result of arbitration of discrepant readings by a third reader in the specific setting of blinded and non-blinded double reading of digital screening mammography.

Our study showed that discrepant readings occurred significantly more often at blinded double reading. Both at blinded and non-blinded double reading, arbitration by a third reader would have resulted in a significantly lower recall rate and significantly higher PPV, without a significant change of the CDR. However, a reduced program sensitivity would have been obtained after arbitration, this effect was statistically significant at blinded double reading. Expressed in numbers, arbitration at blinded double reading would result in $\mathbf{1 8 . 1}$ less recalls per missed cancers and at non-blinded double reading arbitration would result in 19.3 less recalls per missed cancers.

We recently showed that blinded double reading, when compared to non-blinded double reading, resulted in a significantly higher program sensitivity, at the expense of an increased recall rate, a higher proportion of discrepant readings among recalled 
women and an increased false positive recall rate. ${ }^{7}$ An increased false positive recall rate is not desirable as a false positive screen may cause anxiety and psychological distress $^{15-19}$ and possibly changes future screening adherence. ${ }^{20-27}$ Although the recall rates of our study populations at blinded and non-blinded double reading $(3.4 \%$ and $2.8 \%$, respectively) are within the ranges of the European guidelines for quality assurance ${ }^{1}$ and the Dutch optimization study of Otten et al., ${ }^{28}$ it is important to keep the amount of false positives as low as possible. The current study was performed to determine whether the false positive recall could be further reduced through arbitration of discrepant readings by a third reader, without an effect on the CDR and sensitivity. However, our results proved otherwise. Arbitration of discrepant readings by a third reader effectively improves the recall rate and PPV of recall, however at the expense of a reduced the program sensitivity at blinded double reading. Although the effect of arbitration on the program sensitivity at non-blinded double reading is not significant, the program sensitivity following arbitration at non-blinded double reading is more than 10 percent lower compared to blinded double reading without arbitration. Arbitration of discrepant readings is therefore not suitable in our screening population with a recall rate which is well within the ranges of the European guidelines for quality assurance.

A few studies have evaluated the effect of consensus meetings and arbitration on screening outcome at screen film mammography (SFM). Duijm et al. ${ }^{9}$ found that arbitration by a panel of three screening radiologists, performed after persisting reader disagreements between two screening radiologists at consensus meeting, substantially reduced recall rates at discordant reading, at the expense of a small decrease in cancer detection rate. Two Italian studies concluded that arbitration of all discrepant readings at screening mammography by a third reader also substantially reduced recall rates, yields a limited reduction in cancer detection rate and is a cost-effective method to reduce recall rates. ${ }^{10,11}$ Shaw et al. ${ }^{8}$ found that consensus review substantially reduced the number of cases recalled and it was associated with a low false-negative rate. We found a significant reduction in recall rate without a significant influence on the CDR. However, exact comparison of our results is not possible, as none of these studies included the program sensitivity as an outcome measure and all these studies were performed in the era of SFM instead of FFDM.

In our study population, the proportion of BI-RADS 0 among recalls was about $32 \%$, both at blinded and non-blinded double reading. This percentage would have been reduced significantly through arbitration. A Dutch study showed that BI-RADS 0 recalls had a much lower PPV (14.1\%) compared to BI-RADS 4 (39.1\%) and BI-RADS 5 (92.9\%) recalls. ${ }^{29}$ In an attempt to decrease the recall rate, without a detrimental effect on the program sensitivity, it may be worth to determine the effect of arbitration of discrepant BI-RADS 0 rather than arbitration of all discrepant readings. This is a subject of further research.

Our study has certain limitations. We do not provide information on the costeffectiveness of arbitration at blinded versus non-blinded double reading. The lower 
recall rate after arbitration decreases the recall of lesions that turn out to be benign and thus lowers work up costs. ${ }^{30}$ On the other hand, arbitration by a third reader will increase reading-costs of mammography screening and may not be feasible in countries with a shortage of screening radiologists. Although not as efficient as arbitration by a blinded third reader to reduce recall rates, computer-aided detection (CAD) as an arbitrator may be an option to deal with discrepant double reading in the latter countries. ${ }^{31}$ Caumo et al. ${ }^{11}$ showed that arbitration by a third reader is cost-effective in the Italian screening program by counterbalancing excess recall rate at blinded double reading. An additional cost effectiveness analysis will be necessary to determine whether blinded or non-blinded double reading, with or without arbitration of discrepant readings, should become the nationwide standard in the Netherlands.

Our study showed the mammographic abnormalities, tumor characteristics and type of breast surgery of screen detected cancers and cancers missed after arbitration. Unfortunately, the numbers of cancers missed at arbitration were too small to perform further comparisons between blinded and non-blinded double reading.

In conclusion, arbitration of discrepant readings by a third reader is a good tool to improve recall rate and the PPV of recall, but would reduce the program sensitivity at blinded double reading. Arbitration of discrepant readings is therefore not suitable in our screening population with a recall rate, which is well within the ranges of the European guidelines for quality assurance. 


\section{References}

1 Perry N, Broeders M, de Wolf C, Tornberg S, Holland R, von Karsa L. European guidelines for quality assurance in breast cancer screening and diagnosis. Fourth edition--summary document. Ann Oncol 2008;19:614-622.

2 Harvey SC, Geller B, Oppenheimer RG, Pinet M, Riddell L, Garra B. Increase in cancer detection and recall rates with independent double interpretation of screening mammography. AJR Am J Roentgenol 2003;180:1461-1467.

3 Thurfjell EL, Lernevall KA, Taube AA. Benefit of independent double reading in a population-based mammography screening program. Radiology 1994;191:241-244.

4 Brown J, Bryan S, Warren R. Mammography screening: an incremental cost effectiveness analysis of double versus single reading of mammograms. BMJ 1996;312:809-812.

5 Leivo T, Salminen T, Sintonen $\mathrm{H}$ et al. Incremental cost-effectiveness of double-reading mammograms. Breast Cancer Res Treat 1999;54:261-267.

6 Ciatto S, Ambrogetti D, Bonardi R et al. Second reading of screening mammograms increases cancer detection and recall rates. Results in the Florence screening programme. J Med Screen 2005;12:103106.

7 Klompenhouwer EG, Voogd AC, den Heeten GJ et al. Blinded double reading yields a higher programme sensitivity than non-blinded double reading at digital screening mammography: A prospected population based study in the south of The Netherlands. Eur J Cancer 2015;51:391-399.

8 Shaw CM, Flanagan FL, Fenlon HM, McNicholas MM. Consensus review of discordant findings maximizes cancer detection rate in double-reader screening mammography: Irish National Breast Screening Program experience. Radiology 2009;250:354-362.

9 Duijm LE, Groenewoud JH, Hendriks JH, de Koning HJ. Independent double reading of screening mammograms in The Netherlands: effect of arbitration following reader disagreements. Radiology 2004;231:564-570.

10 Ciatto S, Ambrogetti D, Risso $\mathrm{G}$ et al. The role of arbitration of discordant reports at double reading of screening mammograms. J Med Screen 2005;12:125-127.

11 Caumo F, Brunelli S, Tosi $\mathrm{E}$ et al. On the role of arbitration of discordant double readings of screening mammography: experience from two Italian programmes. Radiol Med 2011;116:84-91.

12 Fracheboud J, de Koning $\mathrm{HJ}$, Boer R et al. Nationwide breast cancer screening programme fully implemented in The Netherlands. Breast 2001;10:6-11.

13 Liberman L, Menell JH. Breast imaging reporting and data system (BI-RADS). Radiol Clin North Am 2002;40:409.

14 Sobin LH, Wittekind C. TNM Classification of Malignant Tumours. Wiley-Liss, New York. 2002.

15 Brewer NT, Salz T, Lillie SE. Systematic review: the long-term effects of false-positive mammograms. Ann Intern Med 2007;146:502-510.

16 van der Steeg AF, Keyzer-Dekker CM, De Vries J, Roukema JA. Effect of abnormal screening mammogram on quality of life. Br J Surg 2011;98:537-542.

17 Keyzer-Dekker CM, De Vries J, van Esch L et al. Anxiety after an abnormal screening mammogram is a serious problem. Breast 2012;21:83-88.

18 Bond $\mathrm{M}$, Pavey $\mathrm{T}$, Welch $\mathrm{K}$ et al. Systematic review of the psychological consequences of false-positive screening mammograms. Health Technol Assess 2013;17:1-170, v-vi.

19 Brett J, Austoker J. Women who are recalled for further investigation for breast screening: psychological consequences 3 years after recall and factors affecting re-attendance. J Public Health Med 2001;23:292-300.

20 Klompenhouwer EG, Duijm LE, Voogd AC et al. Re-attendance at biennial screening mammography following a repeated false positive recall. Breast Cancer Res Treat 2014;145:429-437.

21 Lampic C, Thurfjell E, Sjoden PO. The influence of a false-positive mammogram on a woman's subsequent behaviour for detecting breast cancer. Eur J Cancer 2003;39:1730-1737.

22 Alamo-Junquera D, Murta-Nascimento C, Macia F et al. Effect of false-positive results on reattendance at breast cancer screening programmes in Spain. Eur J Public Health 2012;22:404-408.

23 Seigneurin A, Exbrayat C, Labarere J, Delafosse P, Poncet F, Colonna M. Association of diagnostic workup with subsequent attendance in a breast cancer screening program for false-positive cases. Breast Cancer Res Treat 2011;127:221-228. 
24 McCann J, Stockton D, Godward S. Impact of false-positive mammography on subsequent screening attendance and risk of cancer. Breast Cancer Res 2002;4:R11.

25 Drossaert $\mathrm{CH}$, Boer $\mathrm{H}$, Seydel ER. Does mammographic screening and a negative result affect attitudes towards future breast screening? J Med Screen 2001;8:204-212.

26 Pinckney RG, Geller BM, Burman M, Littenberg B. Effect of false-positive mammograms on return for subsequent screening mammography. Am J Med 2003;114:120-125.

27 Roman R, Sala M, De La Vega M et al. Effect of false-positives and women's characteristics on long-term adherence to breast cancer screening. Breast Cancer Res Treat 2011;130:543-552.

28 Otten JD, Karssemeijer N, Hendriks JH et al. Effect of recall rate on earlier screen detection of breast cancers based on the Dutch performance indicators. J Natl Cancer Inst 2005;97:748-754.

29 Timmers JM, van Doorne-Nagtegaal HJ, Zonderland HM et al. The Breast Imaging Reporting and Data System (BI-RADS) in the Dutch breast cancer screening programme: its role as an assessment and stratification tool. Eur Radiol 2012;22:1717-1723.

30 Duijm LE, Groenewoud JH, Fracheboud J et al. Utilization and cost of diagnostic imaging and biopsies following positive screening mammography in the southern breast cancer screening region of the Netherlands, 2000-2005. Eur Radiol 2008;18:2390-2397.

31 James JJ, Cornford EJ. Does computer-aided detection have a role in the arbitration of discordant double-reading opinions in a breast-screening programme? Clin Radiol 2009;64:46-51. 


\section{Chapter 4.4}

Arbitration of discrepant BI-RADS 0 recalls by a third reader at screening mammography lowers recall rate but not the cancer detection rate and sensitivity at blinded and non-blinded double reading

Klompenhouwer EG, Weber RJP, Voogd AC, den Heeten GJ, Strobbe LJA, Broeders MJM, Tjan-Heijnen VCG, Duijm LEM

Breast: 2015 June 24 (epub ahead of print) 


\section{Abstract}

Purpose

To evaluate the characteristics of low suspicion lesions (BI-RADS 0) at blinded and non-blinded double reading of screening mammograms and to determine the potential effect of arbitration of discrepant BI-RADS 0 recalls by a third reader on screening outcome.

\section{Methods}

We included a series of 84,927 consecutive digital screening mammograms, double read in a blinded (43,184 screens) or non-blinded (41,743 screens) fashion, between July 2009 and July 2011. Discrepant readings were routinely recalled for further evaluation. During 2 years of followup, radiology, surgical and pathology reports were collected of all recalled women. Arbitration of discrepant BI-RADS 0 recalls (only one radiologist assigning a BI-RADS 0 score) was retrospectively performed by a third screening radiologist.

\section{Results}

At blinded and non-blinded double reading, $32.0 \%$ and $32.5 \%$ of recalls were assigned BI-RADS 0 with a positive predictive value (PPV) of $7.2 \%$ and $6.8 \%$, respectively. Compared to non-blinded double reading, BI-RADS 0 recalls at blinded double reading showed a higher discrepancy rate (9.0 versus 4.3 per 1,000 screens, $P<0.001)$ and false positive recall rate $(10.1$ versus 8.4 per 1,000 screens, $P=0.012$ ).

Arbitration of discrepant BI-RADS 0 recalls would have significantly lowered recall rate (from $3.4 \%$ to $2.8 \%$ at blinded double reading, $P<0.001$, and from $2.8 \%$ to $2.5 \%$ at non-blinded double reading, $P=0.008$ ), without a decrease in cancer detection rate (from $7.5 \%$ o to $7.3 \%, P=0.751$, and from $6.6 \%$ to $6.5 \%, P=0.832$, respectively) and program sensitivity (from $83.2 \%$ to $81.2 \%$, $P=0.453$, and from $76.0 \%$ to $74.6 \%, P=0.667$, respectively). Arbitration would have significantly increased the PPV at blinded double reading (from $22.3 \%$ to $26.3 \%, P=0.015$ ).

\section{Conclusion}

We advise arbitration of discrepant BI-RADS 0 recalls, at (non-)blinded double reading of screening mammograms, to reduce recall rates and improve the PPV of recall at blinded double reading. 


\section{Introduction}

Screening mammography programs seek to find an optimal balance between recall rate and cancer detection rate. Recall of lesions that turn out to be benign should be avoided as much as possible in order to minimize anxiety among the screened population, to minimize unnecessary work up costs and to maintain the willingness among women to attend future screening rounds. ${ }^{1-4}$

European guidelines consider double reading by screening radiologists as standard of reference for the assessment of screening mammograms as this reading strategy significantly increases the cancer detection rate when compared to single reading., Double reading can be performed either in a blinded fashion $\left(2^{\text {nd }}\right.$ reader is not informed about the $1^{\text {st }}$ reader's decision) or non-blinded fashion $\left(2^{\text {nd }}\right.$ reader is informed about the $1^{\text {st }}$ reader's decision). We recently showed that blinded double reading at full field digital mammography (FFDM) resulted in a significantly better program sensitivity of screening mammography compared to non-blinded double reading, but at the expense of a higher overall recall rate and false positive recall rate. ${ }^{7}$. In that study, the women with a discrepant reading between two screening radiologists (i.e., when only one radiologist considers recall necessary) were always recalled for further analysis.

The Breast imaging Reporting and Data System (BI-RADS) atlas is developed by the American College of Radiology (ACR) to improve communication between physicians, and provides standardized mammographic reporting, breast imaging terminology and a classification system. ${ }^{8}$ BI-RADS category 0 represents a possible finding needing additional work-up, BI-RADS 1 represents a negative finding, BI-RADS 2 represents a benign finding, BI-RADS 4 represents a suspicious abnormality with a reasonable possibility of being malignant but not the classic appearance of malignancy and BI-RADS 5 represents an abnormality highly suggestive for malignancy. ${ }^{9}$ BI-RADS category 3 represents a probably benign finding, needing short interval follow-up. Logistic limitations hamper the incorporation of this latter follow-up strategy in the screening program and therefore the BI-RADS 3 category is not available in the Dutch breast cancer screening program setting.

BI-RADS was introduced nationwide in the Dutch screening program in 2009-2010. A pilot study in the Amsterdam screening region ${ }^{9}$ in the period 2005-2006 showed that BI-RADS was a suitable tool for stratifying the women according to the radiologists' suspicion for malignancy. In the Netherlands, women with a screening BI-RADS 0,4 or 5 are recalled and further evaluated at a dedicated hospital breast unit. BI-RADS category 0 represents an abnormality with low suspicion requiring additional work-up. ${ }^{10}$ These women have a lower positive predictive value (PPV) of screening mammography, (14.1\%) than BI-RADS 4 (39.1\%) and BI-RADS 5 (92.9\%). ${ }^{9}$ Little is known about the mammographic and tumor characteristics of BI-RADS 0 recalls.

The purpose of the current study is twofold: first, to evaluate the PPV, discrepancy rate, and characteristics of BI-RADS 0 recalls in the specific setting of a populationbased digital screening program with blinded and non-blinded double reading; and 
second, to determine the potential effect of arbitration by a third reader of discrepant BI-RADS 0 readings at both reading strategies.

\section{Patients and methods}

\section{Study population}

We included a consecutive series of 84,927 women with a full-field digital screening mammogram, obtained between July 1, 2009 and July 1, 2011. The screens were acquired at three specialized screening units in a southern screening mammography region of the Netherlands (BOZ, BevolkingsOnderzoekZuid).

The women participating in the nationwide Dutch screening program are routinely asked to give permission for the use of their data for scientific purposes. Two women screened at our units did not give this permission and they were excluded. This study was performed under the national permit for breast cancer screening, which is issued by the Ministry of Health, Welfare and Sports after advice of the Dutch Health Council. This study did not require a special permit according to the Dutch Law on Population based screening as both blinded and non-blinded double reading were considered "standard of care" at the time of the study.

\section{Screening procedure and recall}

The nationwide breast cancer screening program of the Netherlands provides free biennial screening mammography for women aged $50-75$ years and is organized by a system of personal invitations with a program compliance rate of $80 \%$.

Details of the screening procedures have been described previously. ${ }^{7}$ In brief, the mammographic examinations were read by a team of 12 certified screening radiologists with 1 to 15 years of screening mammography experience. Each radiologist evaluated at least 6,000 screening mammograms yearly.

Screening mammograms were double read, either in a blinded fashion $\left(2^{\text {nd }}\right.$ reader was not informed about the $1^{\text {st }}$ reader's decision) or non-blinded fashion $\left(2^{\text {nd }}\right.$ reader was informed about the $1^{\text {st }}$ reader's decision). This reading strategy was alternated on a monthly basis. During the study period, the women with discrepant readings between the two screening radiologists at blinded or non-blinded double reading (i.e., only one of the radiologists considered recall necessary) were always recalled for further analysis.

Each screen was classified according to the Breast Imaging Reporting and Data System (BI-RADS). ${ }^{8}$ The most suspicious BI-RADS of two readers was considered the BIRADS at recall. The women with a screening BI-RADS 0,4 or 5 were recalled and further evaluated at a dedicated hospital breast unit. BI-RADS 3 is not available in the Dutch screening setting. At screening, mammographic abnormalities were further classified according to one of the following categories: suspicious mass, suspicious calcifications, 
suspicious mass in combination with calcifications, architectural distortion, asymmetry or other abnormality.

\section{Diagnostic work-up and follow-up of recalled women}

A total of 15 regional and university hospitals were involved in the assessment of recalled women. During a follow-up period of at least two years (until the next biennial screening round), we collected data on diagnostic imaging procedures, clinical data, biopsy specimen and surgical procedures of all recalled women at these hospitals, as well as data on TNM (tumor-node-metastases) classification whenever applicable. ${ }^{11}$ Breast cancers were divided into ductal carcinoma in situ (DCIS) and invasive cancers; lobular carcinoma in situ was considered as non-malignant.

\section{Arbitration of discrepant BI-RADS 0 recalls}

Fur the purpose of the current study, each discrepant BI-RADS 0 reading (i.e., only one screening radiologist had assigned BI-RADS 0 , whereas the other radiologist considered recall not necessary) was re-assessed retrospectively by a third screening radiologist to decide whether or not he/she would have recalled the woman (arbitration). These third readings were randomly assigned to the 12 screening radiologists, so every radiologist performed about 50 third readings. The third reader was aware of the discrepancy but was blinded to the screening outcome of the BI-RADS 0 recall.

\section{Detection of interval cancers}

Interval cancers are primary breast cancers in women with a negative screening test, diagnosed before the next biennial screening invitation or within a time period equal to the biennial screening interval for women who have reached the upper age limit for screening. ${ }^{12}$ Calculation of the program sensitivity of screening strategies requires identification of all interval cancers. As linkage of the screening records to the regional cancer registry was not fully operational yet, we used the following available information to trace interval cancers.

- All radiotherapy reports that were received from the regional radiotherapy institutes concerning women who underwent radiotherapy for breast malignancy and who had participated in the screening program;

- Inquiry about pathology specimens at the various regional pathology laboratories, some months after any hospital had requested the screening mammograms of a woman who had not been referred for further analysis;

- The pathology records that were obtained if a woman cancelled a call for subsequent screening because breast cancer had been diagnosed after a previous negative screen;

- The occasional reports on interval cancers provided by general practitioners or medical specialists to the screening centre.' 


\section{Statistical analysis}

All data were stored in a database and statistics were performed using Statistical Package for Social Science 17.0 (SPSS Inc. Chicago, IL).

A Chi square or Fisher exact test were used to test differences in categorical variables (Table 4.4.1 and 4.4.2). We used the number of true positives per 100 recalls to calculate the PPV of recall of BI-RADS categories.

The overall screening outcome measures of the reading strategies (with and without theoretically arbitration of discrepant BI-RADS 0 recalls) were recall rate (number of recalls per 100 screens), cancer detection rate (number of screen detected cancers per 1,000 screens), sensitivity and PPV of recall (number of screen detected cancers per 100 recalls). The calculation of the sensitivity was based on all interval cancers diagnosed within two years after a negative screening examination, the socalled program sensitivity. This program sensitivity, expressed as a percentage, was calculated by dividing the number of women with screen-detected breast cancer, by the total number of women diagnosed with breast cancer (both interval cancers and screen-detected cancers). For the purpose of this study, cancers not recalled after arbitration by a third reader were calculated as interval cancers. These continuous variables (Table 4.4.3 and 4.4.4) were compared using a double sided t-test for independent samples. A p-value of less than 0.05 was considered to indicate statistical significance.

\section{Results}

\section{BI-RADS categories at recall at blinded and non-blinded double reading without arbitration}

Respectively 43,184 and 41,743 screens had been double read, either in a blinded or non-blinded fashion. The distributions and rates of the BI-RADS categories at recall at blinded and non-blinded double reading are shown in Table 4.4.1.

Of the 1,452 recalls at blinded double reading, 472 were assigned BI-RADS 0 (32.5\%), 921 BI-RADS 4 (63.4\%) and 59 BI-RADS 5 (4.1\%). The PPV of a BI-RADS 0, 4 and 5 recall were respectively $7.2 \%$ (34/472), 25.6\% (235/921) and 91.5\% (54/59).

The cohort of non-blinded double reading comprised 1,188 recalls, of which 380 were assigned BI-RADS 0 (32.0\%), 751 BI-RADS 4 (63.2\%) and 57 BI-RADS 5 (4.8\%). The PPV of a BI-RADS 0, 4 and 5 recall were respectively 6.8\% (26/380), 26.4\% (198/751) and $89.5 \%(51 / 57)$.

Per 1000 screened women, blinded double reading resulted in significantly more BI-RADS category 0 and 4 recalls (10.9 versus 9.1, $P=0.008$ and 21.3 and 18.0, $P<0.001$,Table 4.4.1). 
Table 4.4.1 BI-RADS recall categories at blinded and non-blinded double reading of screening mammograms

\begin{tabular}{|c|c|c|c|c|c|c|c|c|}
\hline & \multicolumn{5}{|c|}{ Proportion } & \multicolumn{3}{|c|}{ Rate (per 1,000 screens) } \\
\hline & \multicolumn{2}{|c|}{ BDR } & \multicolumn{2}{|c|}{ NBDR } & \multirow[t]{2}{*}{$P$-value } & \multirow[t]{2}{*}{ BDR } & \multirow[t]{2}{*}{ NBDR } & \multirow[t]{2}{*}{$P$-value } \\
\hline & $N$ & $\%$ & $N$ & $\%$ & & & & \\
\hline BI-RADS category* & & & & & 0.650 & & & \\
\hline 0 & 472 & 32.5 & 380 & 32.0 & & 10.9 & 9.1 & 0.008 \\
\hline 4 & 921 & 63.4 & 751 & 63.2 & & 21.3 & 18.0 & $<0.001$ \\
\hline 5 & 59 & 4.1 & 57 & 4.8 & & 1.4 & 1.4 & 0.998 \\
\hline
\end{tabular}

* Most suspicious BI-RADS of two readers. BDR blinded double reading; NBDR non-blinded double reading

\section{Characteristics of BI-RADS 0 recalls at blinded and non-blinded without arbitration}

Compared to non-blinded double reading, BI-RADS 0 recalls at blinded double reading were characterized by a higher discrepancy rate at recall (9.0 versus 4.3 per 1,000 screens, $P<0.001)$ and a higher false positive recall rate $(10.1$ versus 8.4 per 1,000 screens, $P=0.012$ ). Both at blinded and non-blinded double reading, about $85 \%$ of the BI-RADS 0 recalls consisted of masses, followed by architectural distortions (about 7\%) and asymmetry (3-4\%). We observed no differences in mammographic and tumor characteristics of screen detected cancers diagnosed after a BI-RADS 0 recall at blinded and non-blinded double reading (Table 4.4.2).

Table 4.4.2 Characteristics of BI-RADS 0 recalls at blinded and non-blinded double reading of screening mammograms

\begin{tabular}{|c|c|c|c|c|c|c|c|c|}
\hline & \multicolumn{5}{|c|}{ Proportion } & \multicolumn{3}{|c|}{$\begin{array}{c}\text { Rate (per } 1000 \text { screened } \\
\text { women) }\end{array}$} \\
\hline & \multicolumn{2}{|c|}{ BDR } & \multicolumn{2}{|c|}{ NBDR } & \multirow[t]{2}{*}{$P$-value } & \multirow[t]{2}{*}{ BDR } & \multirow[t]{2}{*}{ NBDR } & \multirow[t]{2}{*}{$P$-value } \\
\hline & $N$ & $\%$ & $N$ & $\%$ & & & & \\
\hline \multicolumn{9}{|l|}{ BI-RADS category 0} \\
\hline Mammographic abnormality & & & & & 0.509 & & & \\
\hline Mass & 403 & 85.4 & 326 & 85.8 & & 9.3 & 7.8 & 0.016 \\
\hline Suspicious calcifications & 9 & 1.9 & 8 & 2.1 & & 0.2 & 0.2 & 0.863 \\
\hline Mass with calcifications & 3 & 0.6 & 7 & 1.8 & & 0.1 & 0.2 & 0.219 \\
\hline Asymmetry & 20 & 4.2 & 13 & 3.4 & & 0.5 & 0.3 & 0.262 \\
\hline Architectural distortion & 37 & 7.8 & 26 & 6.8 & & 0.9 & 0.6 & 0.211 \\
\hline Discrepant reading* & & & & & $<0.001$ & & & \\
\hline Yes & 388 & 82.2 & 178 & 46.8 & & 9.0 & 4.3 & $<0.001$ \\
\hline No & 84 & 17.8 & 202 & 53.2 & & 1.9 & 4.6 & $<0.001$ \\
\hline Breast cancer & & & & & 0.893 & & & \\
\hline Yes & 34 & 7.2 & 26 & 6.8 & & 0.8 & 0.6 & 0.367 \\
\hline No & 438 & 92.8 & 354 & 93.2 & & 10.1 & 8.4 & 0.012 \\
\hline \multicolumn{9}{|l|}{ Screen detected cancers } \\
\hline Type of screen detected cancer & & & & & 0.076 & & & \\
\hline Ductal carcinoma in-situ & 1 & 2.9 & 5 & 19.2 & & 0.0 & 0.1 & 0.119 \\
\hline Invasive cancer & 33 & 97.1 & 21 & 80.8 & & 0.8 & 0.5 & 0.131 \\
\hline
\end{tabular}


Table 4.4.2 (continued)

\begin{tabular}{|c|c|c|c|c|c|c|c|c|}
\hline & \multicolumn{5}{|c|}{ Proportion } & \multicolumn{3}{|c|}{$\begin{array}{l}\text { Rate (per } 1000 \text { screened } \\
\text { women) }\end{array}$} \\
\hline & \multicolumn{2}{|c|}{ BDR } & \multicolumn{2}{|c|}{ NBDR } & \multirow[t]{2}{*}{$P$-value } & \multirow[t]{2}{*}{ BDR } & \multirow[t]{2}{*}{ NBDR } & \multirow[t]{2}{*}{$P$-value } \\
\hline & $N$ & $\%$ & $N$ & $\%$ & & & & \\
\hline Mammographic abnormality & & & & & 0.497 & & & \\
\hline Mass & 26 & 76.5 & 20 & 76.9 & & 0.6 & 0.5 & 0.441 \\
\hline Suspicious calcifications & 1 & 2.9 & 1 & 3.8 & & 0.0 & 0.0 & 1.000 \\
\hline Mass with calcifications & 0 & 0.0 & 1 & 3.8 & & 0.0 & 0.0 & 0.492 \\
\hline Asymmetry & 0 & 0.0 & 1 & 3.8 & & 0.0 & 0.0 & 0.492 \\
\hline Architectural distortion & 7 & 20.6 & 3 & 11.5 & & 0.2 & 0.1 & 0.344 \\
\hline Invasive cancers & & & & & & & & \\
\hline Size of invasive cancer & & & & & 1.000 & & & \\
\hline $\mathrm{T} 1 \mathrm{a}-\mathrm{c}$ & 27 & 81.8 & 18 & 85.7 & & 0.6 & 0.4 & 0.219 \\
\hline $\mathrm{T} 2+$ & 6 & 18.2 & 3 & 14.3 & & 0.1 & 0.1 & 0.508 \\
\hline Lymph node status & & & & & 0.287 & & & \\
\hline Positive & 4 & 12.1 & 5 & 23.8 & & 0.1 & 0.1 & 0.750 \\
\hline Negative & 29 & 87.9 & 16 & 76.2 & & 0.7 & 0.4 & 0.068 \\
\hline Tumor stage & & & & & 1.000 & & & \\
\hline Advanced** & 8 & 24.2 & 5 & 23.8 & & 0.2 & 0.1 & 0.245 \\
\hline Not-advanced & 25 & 75.8 & 16 & 76.2 & & 0.6 & 0.4 & 0.194 \\
\hline Histology type & & & & & 0.399 & & & \\
\hline Ductal & 27 & 81.8 & 17 & 81.1 & & 0.6 & 0.4 & 0.163 \\
\hline Lobular & 3 & 9.1 & 0 & 0.0 & & 0.1 & 0.0 & 0.250 \\
\hline Mixed ductal/lobular & 2 & 6.1 & 3 & 14.3 & & 0.0 & 0.1 & 0.682 \\
\hline Other & 1 & 3.0 & 1 & 4.8 & & 0.0 & 0.0 & 1.000 \\
\hline Nottingham grade & & & & & 0.481 & & & \\
\hline Nottingham I & 14 & 42.4 & 12 & 57.1 & & 0.3 & 0.3 & 0.760 \\
\hline Nottingham II & 13 & 39.4 & 8 & 38.1 & & 0.3 & 0.2 & 0.311 \\
\hline Nottingham III & 5 & 15.2 & 1 & 4.8 & & 0.1 & 0.0 & 0.219 \\
\hline Unknown & 1 & 3.0 & 0 & 0.0 & & 0.0 & 0.0 & 1.000 \\
\hline Estrogen receptor & & & & & 0.595 & & & \\
\hline Positive & 29 & 87.9 & 20 & 95.2 & & 0.7 & 0.5 & 0.243 \\
\hline Negative & 3 & 9.1 & 1 & 4.8 & & 0.1 & 0.0 & 0.625 \\
\hline Unknown & 1 & 3.0 & 0 & 0.0 & & 0.0 & 0.0 & 1.000 \\
\hline Progesterone receptor & & & & & 0.713 & & & \\
\hline Positive & 25 & 75.8 & 16 & 76.2 & & 0.6 & 0.4 & 0.194 \\
\hline Negative & 7 & 21.2 & 5 & 23.8 & & 0.2 & 0.1 & 0.604 \\
\hline Unknown & 1 & 3.0 & 0 & 0.0 & & 0.0 & 0.0 & 1.000 \\
\hline Her2/Neu receptor & & & & & 0.063 & & & \\
\hline Positive & 0 & 0.0 & 3 & 14.3 & & 0.0 & 0.1 & 0.119 \\
\hline Negative & 32 & 97.0 & 18 & 85.7 & & 0.7 & 0.4 & 0.063 \\
\hline Unknown & 1 & 3.0 & 0 & 0.0 & & 0.0 & 0.0 & 1.000 \\
\hline Triple- receptor negative & & & & & 0.254 & & & \\
\hline Yes & 3 & 9.1 & 0 & 0.0 & & 0.1 & 0.0 & 0.250 \\
\hline No & 29 & 87.9 & 21 & 100 & & 0.7 & 0.5 & 0.312 \\
\hline Unknown & 1 & 3.0 & 0 & 0.0 & & 0.0 & 0.0 & 1.000 \\
\hline
\end{tabular}

$B D R$ blinded double reading; NBDR non-blinded double reading. * Only one screening radiologist had assigned $B I-R A D S 0$, whereas the other radiologist considered recall not necessary. ${ }^{* *}$ Invasive tumor size $>20 \mathrm{~mm}(\mathrm{T2+})$ and/or lymph node positive invasive cancer 


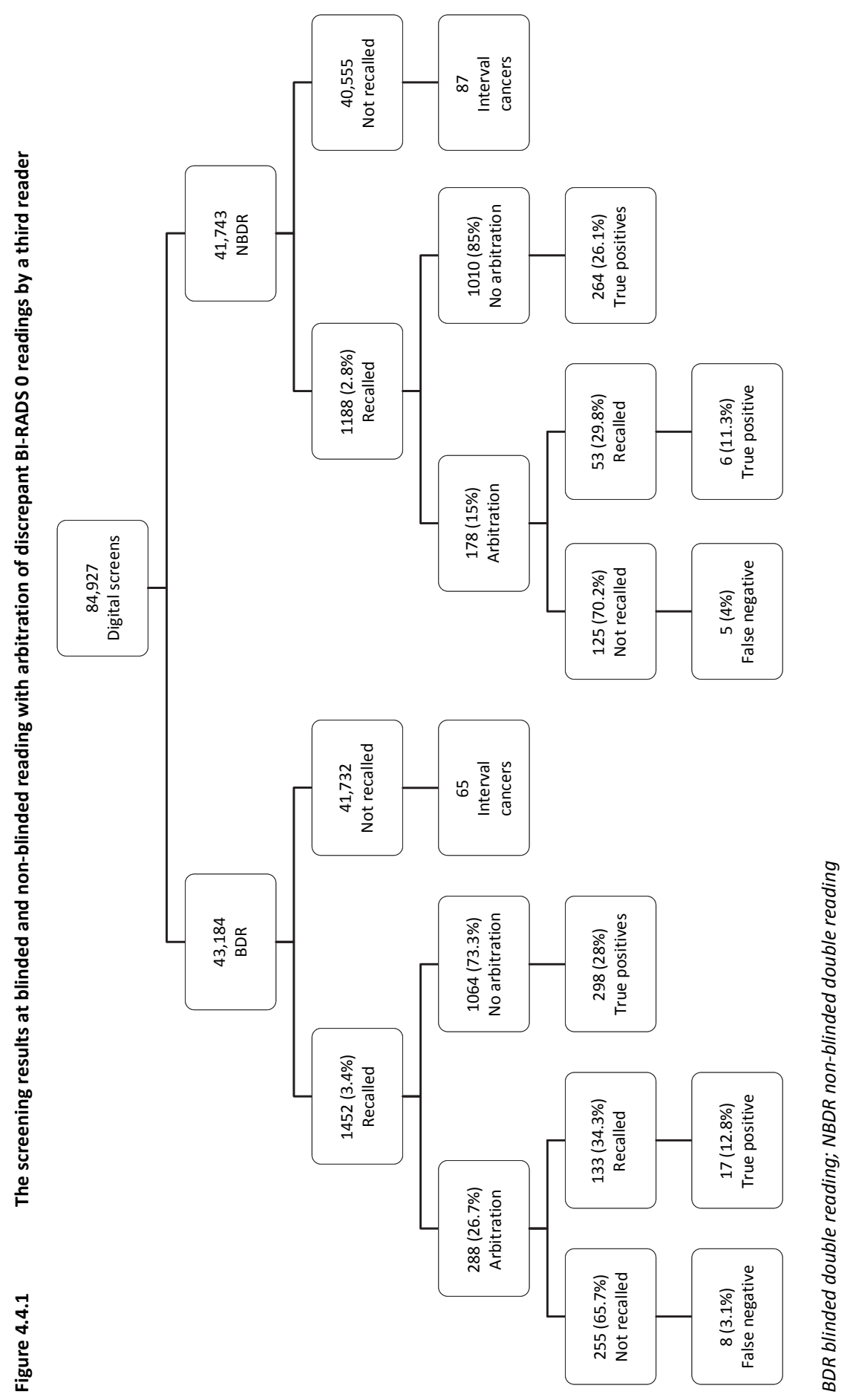




\section{Arbitration of discrepant BI-RADS 0 readings at blinded double reading}

Of the 388 discrepant BI-RADS 0 readings at blinded double reading, 133 (34.3\%) would have been recalled after arbitration, of which 17 (12.8\%) proved to be malignant. Of the remaining 255 screens that would not have been recalled after arbitration, 8 (3\%) proved to be malignant, and these cancers were considered interval cancers for the purpose of this study (Figure 4.4.1). The 8 cancers comprised 6 smaller (T1a-c) and 2 larger (T2) invasive cancers, of which one was lymph node positive at the time of recall.

The overall screening results at blinded double reading, without and with arbitration of discrepant BI-RADS 0 readings by a third reader, are shown in Table 4.4.3. Arbitration would have decreased the recall rate from $3.4 \%$ to $2.8 \%(P<0.001)$ and would have increased the PPV of recall from $22.3 \%$ to $26.3 \%(p=0.015)$. Arbitration would neither have altered the program sensitivity (from $83.2 \%$ to $81.2 \%, P=0.453$ ), nor the cancer detection rate (from 7.5 to 7.3 per 1000 screens, $P=0.751$ ).

Table 4.4.3 Overall screening results of 43,184 blinded double readings at screening mammography, without and with arbitration of discrepant BI-RADS 0 readings by a third reader

\begin{tabular}{lccc} 
& $\begin{array}{c}\text { Recall of all } \\
\text { discrepant readings* }\end{array}$ & $\begin{array}{c}\text { Recall following } \\
\text { arbitration of discrepant } \\
\text { BI-RADS 0 readings }\end{array}$ & $P$-value \\
\hline Recalls, $N$ & 1452 & 1197 & $<0.001$ \\
Recall rate, \% (95\% Cl) & $3.4(3.2-3.5)$ & $2.8(2.6-2.9)$ & 315 \\
Screen detected cancers, $N$ & 323 & $73 * *$ & 0.751 \\
Interval cancers, $N$ & 65 & $7.3(6.5-8.1)$ & 0.015 \\
Cancer detection rate, \%o $(95 \% \mathrm{Cl})$ & $7.5(6.7-8.3)$ & $26.3(23.8-28.8)$ & 0.453 \\
Positive predictive value of recall, \% $(95 \% \mathrm{Cl})$ & $22.3(20.1-24.4)$ & $81.2(77.3-85.1)$ & \\
Program sensitivity, $\%(95 \% \mathrm{Cl})$ & $83.2(79.5-87.0)$ & &
\end{tabular}

* Only one screening radiologist had assigned BI-RADS 0 , whereas the other radiologist considered recall not necessary. ${ }^{* *} 65$ true interval cancers and 8 cancers that would not have been recalled at arbitration

\section{Arbitration of discrepant BI-RADS 0 readings at non-blinded double reading}

Of the 178 discrepant BI-RADS 0 readings at non-blinded double reading, $53(29.8 \%)$ would have been recalled after arbitration, including $6(11.3 \%)$ breast cancers. Of the $125(70.2 \%)$ screens not recalled after arbitration, 5 (4\%) proved to be malignant (1 ductal carcinoma in-situ and 4 invasive cancers (3 T1a-c and 1 T2, of which 1 was lymph node positive).

Table 4.4.4 shows the overall screening results at non-blinded double reading, without and with arbitration of discrepant BI-RADS 0 readings by a third reader. Arbitration would have decreased the recall rate from $2.8 \%$ to $2.5 \%(P=0.008)$, but without an effect on the cancer detection rate (from 6.6 to 6.5 per 1,000 screens, $P=0.832$ ), PPV of recall (from $23.2 \%$ to $25.4 \%(P=0.213$ ) or program sensitivity (from $76.0 \%$ to $74.6 \%, P=0.667)$. 
Table 4.4.4 Screening results of 41,743 non-blinded double readings at screening mammography, without and with arbitration of discrepant BI-RADS 0 readings by a third reader

\begin{tabular}{lccc} 
& $\begin{array}{c}\text { Recall of all discrepant } \\
\text { readings* }\end{array}$ & $\begin{array}{c}\text { Recall following } \\
\text { arbitration of discrepant } \\
\text { BI-RADS 0 readings }\end{array}$ & $P$-value \\
\hline Recalls, $N$ & 1188 & 1063 & 0.008 \\
Recall rate, \% $(95 \% \mathrm{Cl})$ & $2.8(2.7-3.0)$ & $2.5(2.4-2.7)$ & 270 \\
Screen detected cancers, $N$ & 275 & $92 * *$ & 0.832 \\
Interval cancers, $N$ & 87 & $6.5(5.7-7.2)$ & 0.213 \\
Cancer detection rate, \%o $(95 \% \mathrm{Cl})$ & $6.6(5.8-7.4)$ & $25.4(22.8-28.0)$ & 0.667 \\
Positive predictive value of recall, \% $(95 \% \mathrm{Cl})$ & $23.2(20.8-25.6)$ & $74.6(70.1-79.1)$ & 0.67 )
\end{tabular}

* Only one screening radiologist had assigned BI-RADS 0 , whereas the other radiologist considered recall not necessary. ${ }^{* *} 87$ true interval cancers and 5 cancers that would not have been recalled at arbitration

\section{Discussion}

The current study reports the characteristics of BI-RADS 0 recalls and is showing the results of arbitration of discrepant BI-RADS 0 recalls by a third reader, in the specific setting of both blinded and non-blinded double reading of digital screening mammograms.

We found that about $32 \%$ of all recalls were assigned the BI-RADS category 0 both at blinded and non-blinded double reading, whereas the PPV of these recalls was about 7\%. Significantly more BI-RADS 0 recalls consisted of discrepant readings at blinded than at non-blinded double reading. We observed no differences in mammographic and tumor characteristics of screen detected cancers diagnosed after a BI-RADS 0 recall at blinded or non-blinded double reading. Arbitration of discrepant BI-RADS 0 recalls would have significantly lowered the overall recall rate of the screening program without a decrease in overall cancer detection rate and program sensitivity. Arbitration would have significantly increased the PPV at blinded double reading.

We previously reported that blinded double reading increased program sensitivity compared to non-blinded double reading, at the expense of an increased recall rate. ${ }^{7}$. The current study shows that this increased recall rate is due to a higher number of $\mathrm{BI}-$ RADS category 0 and 4 recalls.

Arbitration of discrepant BI-RADS 0 recalls by a third reader would have resulted in two-third less discrepant BI-RADS 0 recalls at the expense of eight missed cancers at blinded double reading and five missed cancers at non-blinded double reading. These 13 cancers represent $36 \%$ (13/36) of all cancers among discrepant BI-RADS 0 recalls. Due to the low PPV of discrepant BI-RADS 0 recalls of $6.4 \%(25 / 388)$ at blinded double and $6.2 \%(11 / 178)$ at non-blinded double reading, the effect of omitting these 13 cancers on the overall decrease in cancer detection rate is very small, namely $0.1-0.2 \%$ at both reading strategies. Further, at blinded double reading, a significantly increased PPV would have been obtained after arbitration by a third reviewer. We 
therefore advocate the use of arbitration of discrepant BI-RADS category 0 recalls at both reading strategies.

A few studies have evaluated the effect of consensus meetings and arbitration on screening outcome at screen film mammography. Duijm et al. ${ }^{13}$ found that arbitration by a panel of three screening radiologists, performed after persisting reader disagreements between two screening radiologists at consensus meeting, substantially reduced recall rates at discordant reading, at the expense of a small decrease in cancer detection rate. Two Italian studies concluded that arbitration of all discrepant readings at screening mammography by a third reader cost-effectively reduced recall rates, and yielded a limited reduction in cancer detection rate. ${ }^{14,15}$ Shaw et al. ${ }^{16}$ concluded that consensus review substantially reduced the number of cases recalled and it was associated with a low false-negative rate. Finally, Hofvind et al. ${ }^{17}$ found that independent (non-blinded) double reading with consensus at mammography screening has the potential to increase the cancer detection rate compared with single reading. To our knowledge, this is the first study reporting on the role of arbitration of discrepant BI-RADS category 0 recalls at digital mammography on overall screening outcome in the specific setting of blinded and non-blinded double reading.

In the Netherlands, women with a positive screening examination are routinely assessed by a multidisciplinary team at a hospital of their choice, regardless of their BI RADS category at recall. Ideally, women with a low suspicion for malignancy, the BI RADS 0 recalls, promptly receive additional imaging at the screening center to minimize extra workload and psychological distress of a positive recall. However, this former strategy is not feasible in the daily practice of the current breast cancer screening organization of the Netherlands. Another way to speed up the workup of BI RADS 0 recalls is assessment of these mammographic abnormalities merely by a radiologist and thus bypassing the use of a multidisciplinary breast care team. This latter recall strategy is currently being explored in a large national multicentre trial, in which not only overall screening outcome is evaluated, but also quality of life, anxiety, psychological consequences of false positive recalls and costs of health care use. ${ }^{18}$ Arbitration of discrepant BI-RADS 0 recalls may prove to be a valid alternative to this recall strategy as arbitration reduces the recall rates at blinded and non-blinded double reading, without influencing the program sensitivity and cancer detection rate, and increases the PPV of recall at blinded double reading.

About $32 \%$ of all recalls were assigned the BI-RADS 0 category. This proportion is substantially lower than the $53 \%$ reported in the Amsterdam screening region in 20052006. ${ }^{9}$ This difference is probably due to the way the final BI-RADS category at recall was determined. In the current study the final BI-RADS at recall was determined by the most suspicious BI-RADS of two readers (both at blinded and non-blinded double reading), without consensus meeting of discrepant readings. In the study of Timmers et al. ${ }^{9}$ screening was performed by non-blinded double reading and the final BI-RADS of discrepant readings was determined at a consensus meeting or by a third reader. 
Our results confirm that the women with BI-RADS 0 recalls have a relatively low risk of having malignancy, with a PPV of screening mammography of approximately $7 \%$. This is lower than the PPV of 14\%, reported in the pilot study in the Amsterdam screening region in the period 2005-2006, ${ }^{9}$ and the PPV of $9 \%$ presented in another Dutch study performed in 2006-2008. ${ }^{19}$ Our lower PPV is probably due to the higher overall recall rate in our study population $(3.4 \%$ at blinded and $2.8 \%$ at non-blinded double reading, compared to $1.7 \%$ in the Amsterdam pilot study). Several factors caused the recall rate to increase in the Dutch screening program in the past decade. First, the Dutch Reference Centre for Screening (LRCB), responsible for ensuring and improving the quality of the Dutch screening program, actively stimulated screening radiologists to increase recall rates of women with subtle abnormalities in order to detect more breast cancers at an earlier stage. ${ }^{20}$ Second, the introduction of digital screening between 2004 and 2010 resulted in a further increase of the recall rates, ${ }^{21,22}$

We found that the vast majority of BI-RADS 0 recalls consisted of masses, with just a small proportion of mammographic abnormalities characterized by architectural distortions or asymmetries. BI-RADS category 0 recalls almost never contained calcifications and were mostly invasive cancers. This observation is easily explained by the fact that ductal carcinoma in-situ is most commonly characterized by suspicious calcifications at mammography and such lesions are classified as BI-RADS 4 or 5 lesions. $^{8}$

A discrepant reading among BI-RADS 0 recalls occurred more often at blinded than at non-blinded double reading, due to the fact that the second reader lacked the first reader's opinion at blinded double reading. Several studies have shown that a substantial inter- and intra-observer variability may exist among radiologists using the BI-RADS lexicon. ${ }^{23-25}$ Training programs in BI-RADS lexicon may probably reduce these variations in the clinical setting, ${ }^{26}$ as well as among screening radiologists. ${ }^{10}$

Our study has certain limitations. For the purpose of the current study, each discrepant BI-RADS 0 reading was re-assessed retrospectively by a third screening radiologist to decide whether or not he/she would have recalled the woman (arbitration). Therefore, the arbiter knew that his or her decision did not have clinical implications for the screening. This lack of clinical implications may have influenced the third reader's decision. Prospective arbitration of discrepant BI-RADS 0 recalls has recently been implemented in our screening routine. These screening results will be available in the near future.

We provide no information on the cost-effectiveness of arbitration of discrepant BI-RADS 0 recalls at blinded versus non-blinded double reading. The lower recall rate after arbitration decreases the recall of lesions that turn out to be benign and thus lowers work up costs. $0^{1}$ On the other hand, arbitration by a third reader may increase reading-costs of mammography screening and may not be feasible in countries with a shortage of screening radiologists.

Our study showed the characteristics of BI-RADS 0 recalls at blinded and nonblinded double reading of screening mammograms. Due to the low PPV of a BI-RADS 0 
recall, the amount of true positives are relatively low, 34 and 26 at blinded and nonblinded reading, respectively. The comparison of the mammographic and tumor characteristics of screen-detected cancers following a BI-RADS 0 recall at blinded or non-blinded double reading is limited by small number of cancers and the low PPV of recall.

In conclusion, we advise arbitration of discrepant BI-RADS 0 recalls, both at blinded and non-blinded double reading of screening mammograms, to reduce recall rates and improve the PPV of recall at blinded double reading. 


\section{References}

1 Duijm LE, Groenewoud JH, Fracheboud J et al. Utilization and cost of diagnostic imaging and biopsies following positive screening mammography in the southern breast cancer screening region of the Netherlands, 2000-2005. Eur Radiol 2008;18:2390-2397.

2 van der Steeg AF, Keyzer-Dekker CM, De Vries J, Roukema JA. Effect of abnormal screening mammogram on quality of life. Br J Surg 2011;98:537-542.

3 Brett J, Austoker J. Women who are recalled for further investigation for breast screening: psychological consequences 3 years after recall and factors affecting re-attendance. J Public Health Med 2001;23:292-300.

4 Klompenhouwer EG, Duijm LE, Voogd AC et al. Re-attendance at biennial screening mammography following a repeated false positive recall. Breast Cancer Res Treat 2014;145:429-437.

5 Duijm LE, Louwman MW, Groenewoud JH, van de Poll-Franse LV, Fracheboud J, Coebergh JW. Interobserver variability in mammography screening and effect of type and number of readers on screening outcome. Br J Cancer 2009;100:901-907.

6 Gur D, Sumkin JH, Hardesty LA et al. Recall and detection rates in screening mammography. Cancer 2004;100:1590-1594.

7 Klompenhouwer EG, Voogd AC, den Heeten GJ et al. Blinded double reading yields a higher programme sensitivity than non-blinded double reading at digital screening mammography: A prospected population based study in the south of The Netherlands. Eur J Cancer 2015;51:391-399.

8 Radiology ACo. ACR BI-RADS ${ }^{\circledR}$ Breast Imaging Reporting and Data System, Breast Imaging Atlas, Mammography. 4th edition. Preston (Va). 2003.

9 Timmers JM, van Doorne-Nagtegaal HJ, Zonderland HM et al. The Breast Imaging Reporting and Data System (BI-RADS) in the Dutch breast cancer screening programme: its role as an assessment and stratification tool. Eur Radiol 2012;22:1717-1723.

10 Timmers JM, van Doorne-Nagtegaal HJ, Verbeek AL, den Heeten GJ, Broeders MJ. A dedicated BI-RADS training programme: effect on the inter-observer variation among screening radiologists. European Journal of Radiology 2012;81:2184-2188.

11 Sobin LH, Wittekind C. TNM Classification of Malignant Tumours. Wiley-Liss, New York. 2002.

12 Perry N, Broeders M, de Wolf C, Tornberg S, Holland R, von Karsa L. European guidelines for quality assurance in breast cancer screening and diagnosis. Fourth edition--summary document. Ann Oncol 2008;19:614-622.

13 Duijm LE, Groenewoud JH, Hendriks JH, de Koning HJ. Independent double reading of screening mammograms in The Netherlands: effect of arbitration following reader disagreements. Radiology 2004;231:564-570.

14 Ciatto S, Ambrogetti D, Risso $\mathrm{G}$ et al. The role of arbitration of discordant reports at double reading of screening mammograms. J Med Screen 2005;12:125-127.

15 Caumo F, Brunelli S, Tosi E et al. On the role of arbitration of discordant double readings of screening mammography: experience from two Italian programmes. Radiol Med 2011;116:84-91.

16 Shaw CM, Flanagan FL, Fenlon HM, McNicholas MM. Consensus review of discordant findings maximizes cancer detection rate in double-reader screening mammography: Irish National Breast Screening Program experience. Radiology 2009;250:354-362.

17 Hofvind S, Geller BM, Rosenberg RD, Skaane P. Screening-detected breast cancers: discordant independent double reading in a population-based screening program. Radiology 2009;253:652-660.

18 Timmers JM, Damen JA, Pijnappel RM et al. Cost-effectiveness of non-invasive assessment in the Dutch breast cancer screening program versus usual care: A randomized controlled trial. Can J Public Health 2014;105:e342-347.

19 Timmers JM, Verbeek AL, IntHout J, Pijnappel RM, Broeders MJ, den Heeten GJ. Breast cancer risk prediction model: a nomogram based on common mammographic screening findings. Eur Radiol 2013;23:2413-2419.

20 Otten JD, Karssemeijer N, Hendriks JH et al. Effect of recall rate on earlier screen detection of breast cancers based on the Dutch performance indicators. J Natl Cancer Inst 2005;97:748-754. 
21 van Luijt PA, Fracheboud J, Heijnsdijk EA, den Heeten GJ, de Koning HJ, National Evaluation Team for Breast Cancer Screening in Netherlands Study G. Nation-wide data on screening performance during the transition to digital mammography: observations in 6 million screens. Eur J Cancer 2013;49: 3517-3525.

22 Nederend J, Duijm LE, Louwman MW, Groenewoud JH, Donkers-van Rossum AB, Voogd AC. Impact of transition from analog screening mammography to digital screening mammography on screening outcome in The Netherlands: a population-based study. Ann Oncol 2012;23:3098-3103.

23 Ciatto S, Houssami N, Apruzzese A et al. Reader variability in reporting breast imaging according to $\mathrm{BI}-$ RADS assessment categories (the Florence experience). Breast 2006;15:44-51.

24 Cosar ZS, Cetin M, Tepe TK, Cetin R, Zarali AC. Concordance of mammographic classifications of microcalcifications in breast cancer diagnosis: Utility of the Breast Imaging Reporting and Data System (fourth edition). Clin Imaging 2005;29:389-395.

25 Berg WA, Campassi C, Langenberg P, Sexton MJ. Breast Imaging Reporting and Data System: inter- and intraobserver variability in feature analysis and final assessment. AJR Am J Roentgenol 2000;174: 1769-1777.

26 Berg WA, D'Orsi CJ, Jackson VP et al. Does training in the Breast Imaging Reporting and Data System (BIRADS) improve biopsy recommendations or feature analysis agreement with experienced breast imagers at mammography? Radiology 2002;224:871-880. 


\section{Chapter 5}

Discussion and future perspectives: strategies for improvement of population based breast cancer

screening 
There is an ongoing debate on the effectiveness and efficacy of breast cancer screening. In 2001 the Cochrane Collaboration published a meta-analysis in which it was concluded that mammography screening does not improve survival. ${ }^{1}$ In 2006, the same Cochrane Collaboration nuanced their conclusions, stating that breast cancer screening is likely to reduce breast cancer mortality, with a relative risk reduction of $15 \%{ }^{2}$ In an attempt to end the discussion, an independent panel was convened in the United Kingdom to reach conclusions about the benefits and harms of breast cancer screening. The panel estimated that screening reduces breast cancer mortality by about $20 \%$, but that overdiagnosis occurs in approximately $11-19 \%$. They concluded that breast cancer screening should be continued in the United Kingdom, but that this information on the benefits as well as the drawbacks of screening should be made available to women invited to screening so that they can make informed decisions. ${ }^{3}$ The debate about the effects of screening was stirred up again after the publication of a Canadian trial, which showed no reduction in breast cancer mortality, after 25 years of follow-up, in the screened population when compared to the control group. ${ }^{4}$ These results were heavily debated because of the questionable mammographic technology used in this trial.

Recently, a committee of the Health Council of the Netherlands concluded that, in the Netherlands, population screening for breast cancer continues to be as effective as was originally expected, even though the therapeutic landscape has changed significantly. ${ }^{5}$ To continue to keep the current balance in favor of screening one should try to increase the effectiveness of the breast screening program in the Netherlands and minimize the harms.

Screening can only be effective if certain criteria are met, including a sufficiently high attendance, referral- and detection rate, a sufficiently high sensitivity and specificity of the screening test and a relatively low rate of interval cancers and false positive screening results. Based on the results of the studies presented in this thesis and the future perspectives, certain strategies are proposed that may improve the effectiveness of the population based breast cancer program.

\section{Availability of prior hard copy screen films for comparison}

The introduction of digital mammography in the Netherlands was accompanied by higher cancer detection rates at the expense of higher recall rates and a decreased positive predictive value of recall. ${ }^{6-9}$ In chapter $2^{10}$ we showed that, during the transition to digital screening, the availability of prior hard copy screen films for comparison with the findings at digital screening would have reduced the amount of repeated false positives for the same mammographic abnormality by $40 \%$. Further, we found that patients with a repeated false positive recall for the same mammographic lesion were significantly less likely to re-attend the screening program (44.3\%) than 
patients with a negative screen examination (93.2\%) or a single false positive recall (65.4\%).

A previous study from our screening region showed that almost one third of the women who experienced a false positive screen underwent surveillance mammography outside the screening program at the time they were scheduled for subsequent screening mammography. ${ }^{11}$ The decreased screening participation in our study population may be due in part to the same tendency to keep women under outpatient supervision after a repeated false positive recall. Prolonged clinical surveillance of women with a previous false positive screen results in additional diagnostic and followup costs, compared to mammography performed within the national screening program, and is thus not desirable.

For women attending digital mammography screening for the first time after previous screen-film mammography screening and for whom a repeated recall is considered, we therefore recommend that the screening radiologist has the opportunity to compare the digital mammogram with the previous hard copy screen film examination. Failure to do so will increase the amount of false positive screens and eventually lower the re-attendance rate and the effectiveness of screening mammography and will increase the costs related to prolonged clinical surveillance. Although FFDM screening has replaced SFM screening in most breast cancer screening programs, the availability of the previous hard copy screen films remains of value for women who only have SFM screening examinations for comparison. For example, comparison with previous screen films may be of value in screening programs currently converting to digital mammography in foreign countries or where a woman has skipped the digital screening rounds following their latest SFM screen.

\section{Creating specific pairs of screening radiologists}

Substantial inter-observer variability in screening mammography interpretation among screening radiologists has been well documented. ${ }^{12-14}$ In chapter $3^{15}$ we determined the variation in screening performance among unique pairs of screening radiologists at non-blinded double reading of mammograms. We expected that the addition of a second reader would decrease this variation in screening outcome, especially among a group of screening radiologists who attend quality assurance sessions at regular intervals. Nevertheless, our results proved otherwise. Screening performance significantly varied among 26 pairs of screening radiologists with respect to recall rate, program and mammographic sensitivity and the PPV of recall.

The causes for variability among radiologists in screening outcome are not completely clear yet. Several studies have shown that performance of radiologists may improve with screening volumes up to approximately 3000 mammograms interpreted annually, ${ }^{16,17}$ while others found no evidence that greater volumes improves performance. ${ }^{18,19}$ In the Netherlands, all radiologists perform at least 3000 screens per 
year. Therefore, the minimum number of interpreted mammograms may not be an important parameter to explain screening performance. One study even showed that a greater screening focus (more screening/less diagnostic work-up) lowers the cancer detection rates. ${ }^{16}$ It is unknown if extremely high annual volumes (e.g. over 6000 mammograms interpreted annually) will influence screening outcome. This is a subject of current research performed by the Dutch reference centre for screening.

In chapter $3^{15}$ we found significantly higher sensitivities in three screening pairs, and each of these pairs consisted of at least one dedicated breast radiologist. Furthermore, significantly lower sensitivities were observed in two screening pairs, both consisting of one radiologist not involved in diagnostic breast imaging and another radiologist admittedly involved in diagnostic breast imaging, but not being a dedicated breast radiologist. Literature shows that radiologist's performance may be related to the participation in diagnostic work-up ${ }^{16}$ or fellowship training in breast imaging. ${ }^{20}$

To optimize screening outcome it may be desirable to create specific pairs of radiologists in which radiologists with good to superior screening results are combined with radiologists with less optimal screening results or in which radiologists specialized in breast imaging are combined with radiologists who are not (yet) specialized.

\section{Monitoring of the screening results on a local and individual level}

The Dutch reference centre for screening is responsible for ensuring and improving the quality of the breast cancer screening program. Strict rules apply for screening radiologists with respect to additional training and $\mathrm{CME}$-credits in breast radiology. Currently, teams of Dutch screening radiologists receive feedback through medical audits that are performed every 3 years. At these audits, various screening outcome parameters are evaluated, including the cancer detection rate and interval cancer rate. During these meetings a set of 40 interval cancers are re-assessed by breast cancer screening experts to determine whether these interval cancers had been missed, showed a minimal sign or were occult at the last screen. In addition, 40 advanced screen detected cancers (invasive cancers with TNM stage T2a or higher, or the presence of axillary metastasis ${ }^{21}$ ) are evaluated to determine if they could have been recalled at the previous screen. Finally, 40 consecutively recalled women are discussed to determine if the expert panel would have recalled them as well and assigned the same BI-RADS category as had been done by the two screening radiologists.

Despite all these efforts, it appeared that in the period 1997-2011 12.4\% (range $3.4 \%-28 \%$ ) of all cancers were missed at screening (chapter $3^{15}$ ). A missed interval cancer may be related to a failure in the recognition or interpretation of mammographic findings. Therefore, continuous efforts should be made to improve the performance of individuals and pairs of screening radiologists. The analysis of missed cancers and false positive recalls at screening mammography is an important 
component of this quality assurance program. A radiologist who interprets screening examinations without the opportunity to get feedback on the outcome of recalled women may be hampered to improve his/her recall behavior.

Currently, screening results are measured on a regional scale for teams of Dutch screening radiologists by the Dutch reference centre for screening. To detect suboptimal results of screening radiologists or pairs of screening radiologists, it is important to monitor screening results on an individual basis. The digitization of the screening program and the recently established linkage with the Dutch Cancer Registry database provides more opportunities to monitor screening results at an individual basis. The LRCB recently developed a self-test which enables Dutch screening radiologists to evaluate their performance at an individual level. ${ }^{22}$ This test might further improve the quality of breast cancer screening in the Netherlands.

\section{Blinded double reading with arbitration of discrepant BI-RADS 0 recalls}

Double reading can be performed in a blinded or non-blinded fashion and generates discrepant readings among the two reading radiologists. The Dutch Health Council of the Netherlands recommends the use of blinded double reading in screening. ${ }^{5}$ However, very few data report on the advantages and disadvantages of blinded double reading compared to non-blinded double reading ${ }^{23}$ and these two reading strategies have never been compared in a screening setting. In the Netherlands, mammograms were usually read in a non-blinded fashion in the era of screen-film mammography. With the introduction of full-field digital mammography (FFDM) it became technically feasible to perform blinded double reading as an alternative to non-blinded double reading.

In this thesis we evaluated the screening outcomes of both blinded and nonblinded double reading and we explored which of the following three strategies is the best to cope with discrepant readings: recall of all discrepant readings, arbitration of all discrepant readings by a third reader, or arbitration of discrepant BI-RADS 0 recalls only by a third reader. The main screening results of the three reading strategies are presented in table 5.1 .

In chapter $4.1^{24}$ we determined screening mammography outcomes at blinded versus non-blinded double reading. In this study, discrepant readings among the two screening radiologists were always recalled for further evaluation. Blinded double reading yielded a significantly better program sensitivity than non-blinded double reading, as well as a $14 \%$ higher (7.4 versus 6.5 ), although non-significant, cancer detection rate (CDR). However, blinded double reading almost doubled the proportion of discrepant readings among recalled women in our study, which resulted in a significantly higher recall rate and a lower positive predictive value of recall (PPV). 
Although the recall rates at both blinded and non-blinded double reading (3.4\% and $2.8 \%$, respectively) were within the ranges of the European guidelines for quality assurance $^{25}$ and the Dutch optimization study of Otten et al., ${ }^{26}$ it is important to keep the number of false positive test results as low as possible to avoid anxiety and psychological distress among recalled women. ${ }^{27-30}$ The degree of distress following a false positive recall is related to the invasiveness of the tests used in the diagnostic work-up $^{29}$ and in chapter $\mathbf{4 . 2}$ we showed that blinded double reading resulted in a significantly higher benign biopsy rate.

The increased recall rate at blinded double reading compared to non-blinded double reading was due to increased numbers of BI-RADS category 0 and 4 recalls and an increased number of discrepant readings (chapter 4.3). In order to decrease the recall rate and false positive rate, we further determined the potential effect of arbitration of all discrepant readings by a third reader in chapter 4.3 and only discrepant BI-RADS 0 recalls in chapter 4.4. The screening results of these different reading strategies at blinded and non-blinded double reading are shown in Table 5.1.

Table 5.1 Screening results of different reading strategies at blinded and non-blinded double reading

\begin{tabular}{|c|c|c|c|c|c|c|}
\hline Reading strategy & $\begin{array}{c}\text { BDR: } \\
\text { Recall of all } \\
\text { discrepant } \\
\text { readings }\end{array}$ & $\begin{array}{l}\text { BDR: } \\
\text { Arbitration } \\
\text { of all } \\
\text { discrepant } \\
\text { readings }\end{array}$ & $\begin{array}{c}\text { BDR: } \\
\text { Arbitration } \\
\text { of } \\
\text { discrepant } \\
\text { BI-RADS O } \\
\text { readings }\end{array}$ & $\begin{array}{l}\text { NBDR: } \\
\text { Recall of all } \\
\text { discrepant } \\
\text { readings }\end{array}$ & $\begin{array}{l}\text { NBDR: } \\
\text { Arbitration } \\
\text { of all } \\
\text { discrepant } \\
\text { readings }\end{array}$ & $\begin{array}{c}\text { NBDR: } \\
\text { Arbitration } \\
\text { of } \\
\text { discrepant } \\
\text { BI-RADS O } \\
\text { readings }\end{array}$ \\
\hline Recall rate (\%) & 3.4 & 2.2 & 2.8 & 2.8 & 2.3 & 2.5 \\
\hline Cancer detection rate (\%o) & 7.5 & 6.8 & 7.3 & 6.6 & 6.3 & 6.5 \\
\hline Interval cancer rate (\%) & 1.5 & 2.2 & 1.7 & 2.1 & 2.4 & 2.2 \\
\hline Positive predictive value (\%) & 22.3 & 31.2 & 26.3 & 23.2 & 27.5 & 25.4 \\
\hline Program sensitivity (\%) & 83.2 & 76.0 & 81.2 & 76.0 & 72.7 & 74.6 \\
\hline
\end{tabular}

BDR blinded double reading; NBDR non-blinded double reading. * Screening outcomes as mentioned in chapter 4.2 and 4.3

Arbitration of all discrepant readings effectively reduced the recall rate and improved the PPV at both reading strategies. Nevertheless, this reading strategy is not desirable as it also reduced the program sensitivity, and this effect was statistically significant at blinded double reading. Arbitration of all discrepant readings at blinded double reading would have resulted in a program sensitivity that is comparable to that obtained by non-blinded double reading with recall of all discrepant readings $(76 \%$, table 5.1), and thereby neutralizes the positive effect on the program sensitivity of blinded double reading.

On the contrary, arbitration of only discrepant BI-RADS category 0 recalls, would have resulted in a decreased recall rate with both reading strategies, an increased PPV at blinded double reading, without a significant effect on the program sensitivity and cancer detection rate. 
Based on our findings we advocate the use of blinded double reading with arbitration of discrepant BI-RADS 0 recalls in the Netherlands. This reading strategy was shown to provide a high program sensitivity of $81.2 \%$, a cancer detection rate of 7.3 per 1000 screens, a recall rate of $2.8 \%$ and a PPV of recall of $26.3 \%$. Before the introduction of digital screening, we used non-blinded double reading with recall of all discrepant readings as the preferred reading strategy in our screening region. Per 1000 screened women, blinded double reading with arbitration of discrepant BI-RADS category 0 recalls, would result in 0.7 more screen detected cancers and 0.4 less interval cancers compared to non-blinded double reading, without a noticeable effect on the overall recall rate (table 5.1 ).

\section{Alternative to blinded double reading with arbitration of discrepant $\mathrm{BI}$ - RADS 0 recalls}

In the Netherlands, women with a positive screening examination are routinely assessed by a multidisciplinary team at a hospital of their choice, regardless of their BIRADS category at recall (see Figure 1.3, chapter 1 ). Ideally, women with a low suspicion for malignancy, the BI-RADS 0 recalls, promptly receive additional imaging at the screening center to minimize extra workload and psychological distress of a positive recall. However, this strategy is not feasible in the daily practice of the Dutch breast cancer screening program because further diagnostic assessment is not included in the Dutch screening setting. Another possibility to speed up the workup of BI-RADS 0 recalls is the assessment of these mammographic abnormalities merely by a radiologist and thus bypassing the use of a multidisciplinary breast cancer team. This recall strategy has been explored in a large national multicentre trial. The researchers found that it was efficient and cost-saving, without a significant effect on the quality of life. ${ }^{31}$ In this thesis we have shown that blinded double reading with arbitration of discrepant BI-RADS 0 readings could be a valid alternative to the assessment of BI-RADS 0 recalls by merely a radiologist instead of a multidisciplinary team.

\section{Applicability of blinded double reading with arbitration of discrepant BI- RADS 0 recalls}

BI-RADS category 0 assessments in the Netherlands are assigned to findings for which additional mammography views, ultrasound, or previous diagnostics are necessary to assign a final assessment category. The definition of BI-RADS 0 differs worldwide. The latest guidelines of the American College of Radiology (ACR 2008, amended in 2014) recommend that all patients with screening abnormalities should be given a BI-RADS category 0 and that the use of BI-RADS category 4 and 5 is not recommended in the breast cancer screening setting. ${ }^{32}$ This ACR-guideline is designed for use in the United States (US) breast cancer screening service, where most screening facilities have the capacity to instantly perform the recommended examination, for example an additional ultrasound. ${ }^{32}$ The US screening service differs significantly of the Dutch screening 
service, where women with a positive screening examination are routinely assessed by a multidisciplinary team in a hospital. Therefore, the Dutch screening program was not able to adopt the latest ACR-guideline and the BI-RADS 2003 lexicon is used for screening in the Netherlands instead if suspect for malignancy. ${ }^{33}$.

Due to the differences in the use of the term 'BI-RADS category 0 recall', the recommended reading strategy, blinded double reading with arbitration of discrepant BI-RADS 0 recalls, is not automatically applicable in countries where the breast cancer screening organization is different from the situation in the Netherlands. However, in other terms this strategy might also be described as: 'blinded double reading with arbitration of low suspicion lesions by a third reader' and this strategy could be adopted worldwide.

\section{Future perspectives}

\section{Cost-effectiveness analysis}

Our study does not provide information on the cost-effectiveness of the evaluated reading strategies. One may suggest that screening in a blinded fashion increases screening time, as the second reader 'lacks' the first reader's opinion. On the other hand, reading of screening mammograms can be speeded up if the second reader does not have to take the other reader's opinion into account. Further, arbitration by a third reader may increase reading-costs of mammography screening and may also not be feasible in countries with a shortage of screening radiologists. A cost-effectiveness analysis is recommended to determine whether blinded double reading, combined with arbitration of discrepant BI-RADS 0 recalls, should become the nationwide reading strategy in the Netherlands.

\section{A personalized risk-based approach}

All the studies included in this thesis are performed with a 'one-size fits-all' strategy in breast cancer screening. This implies that all women aged 50-75 years are offered the same screening procedure, regardless of their individual risk of developing breast cancer. A more personalized risk-based approach could optimize the efficacy of breast cancer screening, and this issue is currently being investigated in the PRISMA study. ${ }^{34}$

A personalized risk-based approach may be of particular value in patients with dense breast tissue. In Chapter $\mathbf{3}$ we confirmed that the breast density of patients with a missed (interval) cancer was significantly higher compared to patients with a screen detected cancer. The type of supplemental screening, if any, that should be recommended for women with dense breasts, is unclear yet. Supplemental screening by ultrasonography (US) or magnetic resonance (MR) may increase cancer detection in women with dense breasts in combination with at least one other risk factor, but often at the expense of more false-positive findings and benign biopsy results. ${ }^{35,36}$ The added 
value of $M R I$ is undisputed in high risk groups, such as BRCA $1 / 2$ mutation carriers or women with a very strong family history of breast cancer up to the aged of $60 . .^{37,38}$ Currently, a Dutch study group is investigating the role of screening MRI in patients with dense breast tissue. ${ }^{39}$ Breast tomosynthesis combined with digital mammography has shown improvements in false-positive and false-negative screening results ${ }^{40}$ and increases cancer detection rates while decreasing recall rates. ${ }^{41,42}$ This technique may be of particular value in women with dense breasts, as tomosynthesis partially overcomes the masking effect observed at mammography. ${ }^{43}$ However, all types of supplemental screening methods cost time and money. Proper research is therefore necessary prior to adopting one of these additional screening methods.

\section{Conclusion}

Breast cancer remains an important public health threat. Like many other countries, the Netherlands has introduced a population based screening program with the aim to reduce breast cancer mortality by detecting the disease at an early stage. To maintain the effectiveness of breast cancer screening in the rapidly changing therapeutic landscape, it is relevant to continuously evaluate the screening program and try to maximize its benefits and minimize the drawbacks of mass screening. Based on the studies presented in this thesis, the following strategies may improve the effectiveness of the population based breast cancer screening program:

1. If a woman attends digital screening for the first time after previous screen film screening (i.e. screening programs currently converting to digital mammography and women who have skipped the digital screening rounds following their latest SFM screen) the screening radiologist should have the opportunity to compare with the previous hard copy SFM examination if repeated recall is considered.

2. Create specific pairs of radiologists in which radiologists with good to superior screening results are combined with radiologists with less optimal screening results or in which radiologists specialized in breast imaging are combined with radiologists who are not.

3. To detect suboptimal individual screening results or suboptimal results of pairs of screening radiologists, it is important to monitor screening results at a local and individual level.

4. Although a cost effectiveness analysis is still required, blinded double reading with arbitration of discrepant BI-RADS 0 recalls seems the optimal reading strategy to improve the effectiveness of the breast cancer screening program in the Netherlands.

Additionally, a personalized risk-based approach, especially for patients with dense breast tissue, may further improve the efficacy of breast cancer screening in the near future. 


\section{References}

1 Olsen O, Gotzsche PC. Screening for breast cancer with mammography. Cochrane Database Syst Rev: 2001;CD001877.

2 Gotzsche PC, Nielsen M. Screening for breast cancer with mammography. Cochrane Database Syst Rev: 2006;CD001877.

3 Independent UKPoBCS. The benefits and harms of breast cancer screening: an independent review. Lancet 2012;380:1778-1786.

4 Miller AB, Wall C, Baines CJ, Sun P, To T, Narod SA. Twenty five year follow-up for breast cancer incidence and mortality of the Canadian National Breast Screening Study: randomised screening trial. BMJ 2014;348:g366.

5 Population screening for breast cancer: expectations and developments. Heath Council of the Netherlands. 2014.

6 Nederend J, Duijm LE, Louwman MW, Groenewoud JH, Donkers-van Rossum AB, Voogd AC. Impact of transition from analog screening mammography to digital screening mammography on screening outcome in The Netherlands: a population-based study. Ann Oncol 2012;23:3098-3103

7 Bluekens AM, Karssemeijer N, Beijerinck D et al. Consequences of digital mammography in populationbased breast cancer screening: initial changes and long-term impact on referral rates. Eur Radiol 2010;20:2067-2073

8 van Luijt PA, Fracheboud J, Heijnsdijk EA, den Heeten GJ, de Koning HJ. Nation-wide data on screening performance during the transition to digital mammography: Observations in 6 million screens. Eur J Cancer 2013;49:3517-3525

9 National Evaluation Team for Breast Cancer Screening in Netherlands Study G. National evaluation of breast cancer screening the Netherlands 1990-2011/2012. In: Erasmus MC R, (ed). NETB. 2014.

10 Klompenhouwer EG, Duijm LE, Voogd AC et al. Re-attendance at biennial screening mammography following a repeated false positive recall. Breast Cancer Res Treat 2014;145:429-437

11 Setz-Pels W, Duijm LE, Coebergh JW, Rutten M, Nederend J, Voogd AC. Re-attendance after falsepositive screening mammography: a population-based study in the Netherlands. Br J Cancer 2013;109: 2044-2050

12 Elmore JG, Wells CK, Lee $\mathrm{CH}$, Howard DH, Feinstein AR. Variability in radiologists' interpretations of mammograms. N Engl J Med 1994;331:1493-1499

13 Beam C, Sullivan D. Variability in mammogram interpretation. Adm Radiol J 1996;15:47, 49-50, 52

14 Duijm LE, Louwman MW, Groenewoud JH, van de Poll-Franse LV, Fracheboud J, Coebergh JW. Interobserver variability in mammography screening and effect of type and number of readers on screening outcome. Br J Cancer 2009;100:901-907

15 Klompenhouwer EG, Duijm LE, Voogd AC et al. Variations in screening outcome among pairs of screening radiologists at non-blinded double reading of screening mammograms: a population-based study. Eur Radiol 2014;24:1097-1104

16 Buist DS, Anderson ML, Haneuse SJ et al. Influence of annual interpretive volume on screening mammography performance in the United States. Radiology 2011;259:72-84

17 Theberge I, Chang SL, Vandal N et al. Radiologist interpretive volume and breast cancer screening accuracy in a Canadian organized screening program. J Natl Cancer Inst 2014;106:djt461

18 Barlow WE, Chi C, Carney PA et al. Accuracy of screening mammography interpretation by characteristics of radiologists. J Natl Cancer Inst 2004;96:1840-1850

19 Haneuse S, Buist DS, Miglioretti DL et al. Mammographic interpretive volume and diagnostic mammogram interpretation performance in community practice. Radiology 2012;262:69-79

20 Elmore JG, Jackson SL, Abraham L et al. Variability in interpretive performance at screening mammography and radiologists' characteristics associated with accuracy. Radiology 2009;253:641-651

21 AJCC Cancer Staging Manual, Seventh Edition. Springer, New York. 2010.

22 Timmers JM, Verbeek AL, Pijnappel RM, Broeders MJ, den Heeten GJ. Experiences with a self-test for Dutch breast screening radiologists: lessons learnt. Eur Radiol 2014;24:294-304

23 Groenewoud JH, Otten JD, Fracheboud J et al. Cost-effectiveness of different reading and referral strategies in mammography screening in the Netherlands. Breast Cancer Res Treat 2007;102:211-218 
24 Klompenhouwer EG, Voogd AC, den Heeten GJ et al. Blinded double reading yields a higher programme sensitivity than non-blinded double reading at digital screening mammography: A prospected population based study in the south of The Netherlands. Eur J Cancer 2015;51:391-399

25 Perry N, Broeders M, de Wolf C, Tornberg S, Holland R, von Karsa L. European guidelines for quality assurance in breast cancer screening and diagnosis. Fourth edition--summary document. Ann Oncol 2008;19:614-622

26 Otten JD, Karssemeijer N, Hendriks JH et al. Effect of recall rate on earlier screen detection of breast cancers based on the Dutch performance indicators. J Natl Cancer Inst 2005;97:748-754

27 van der Steeg AF, Keyzer-Dekker CM, De Vries J, Roukema JA. Effect of abnormal screening mammogram on quality of life. Br J Surg 2011;98:537-542

28 Brewer NT, Salz T, Lillie SE. Systematic review: the long-term effects of false-positive mammograms. Ann Intern Med 2007;146:502-510

29 Bond M, Pavey T, Welch K et al. Psychological consequences of false-positive screening mammograms in the UK. Evid Based Med 2013;18:54-61

30 Brodersen J, Siersma VD. Long-term psychosocial consequences of false-positive screening mammography. Ann Fam Med 2013;11:106-115

31 Timmers JM, Damen JA, Pijnappel RM et al. Cost-effectiveness of non-invasive assessment in the Dutch breast cancer screening program versus usual care: A randomized controlled trial. Can J Public Health 2014;105:e342-347

32 American American College of R (2008, Amended 2014) ACR practice guideline for the performance of screening and diagnostic mammography. Preston (Va)

33 Radiology ACo. ACR BI-RADS ${ }^{\circledR}$ Breast Imaging Reporting and Data System, Breast Imaging Atlas, Mammography. 4th edition. Preston (Va). 2003.

34 Broeders JM. Breast cancer screening-from one-size-fits-all to a personalized risk-based approach. Available via http://www.zonmw.nl/nl/projecten/project-detail/breast-cancer-screening-from-one-sizefits-all-to-a-personalised-risk-based-approach/samenvatting/. 2013

35 Berg WA, Blume JD, Cormack JB et al. Combined screening with ultrasound and mammography vs mammography alone in women at elevated risk of breast cancer. JAMA 2008;299:2151-2163

36 Berg WA, Zhang Z, Lehrer D et al. Detection of breast cancer with addition of annual screening ultrasound or a single screening MRI to mammography in women with elevated breast cancer risk. JAMA 2012;307:1394-1404

37 Warner E, Messersmith H, Causer P, Eisen A, Shumak R, Plewes D. Systematic review: using magnetic resonance imaging to screen women at high risk for breast cancer. Ann Intern Med 2008;148:671-679

38 Rutgers EJ, Nortier JW, Tuut MK et al. [Dutch Institute for Healthcare Improvement guideline, "Treatment of breast cancer"]. Ned Tijdschr Geneeskd 2002;146:2144-2151

39 van Gils $\mathrm{CH}$, Veldhuis W, Peeters P. [Tailored breast cancer screening with ultrasound and MRI?]. Ned Tijdschr Geneeskd 2012;156:A5313

40 McCarthy AM, Kontos D, Synnestvedt $M$ et al. Screening outcomes following implementation of digital breast tomosynthesis in a general-population screening program. J Natl Cancer Inst 2014;106

41 Ciatto S, Houssami N, Bernardi D et al. Integration of 3D digital mammography with tomosynthesis for population breast-cancer screening (STORM): a prospective comparison study. Lancet Oncol 2013;14:583-589

42 Destounis SV, Morgan R, Arieno A. Screening for dense breasts: digital breast tomosynthesis. AJR Am J Roentgenol 2015;204:261-264

43 Baker JA, Lo JY. Breast tomosynthesis: state-of-the-art and review of the literature. Academic Radiology 2011;18:1298-1310 


\section{Chapter 6.1}

Summary 


\section{General introduction}

Breast cancer is the most frequently diagnosed cancer in women worldwide. Also in the Netherlands, breast cancer is an important threat for public health as the incidence is among the highest in Europe, and still rising. As in many other countries, the Netherlands has introduced a population based screening program, with the aim to reduce breast cancer mortality by detecting the disease at an early stage.

Screening can only be effective if certain criteria are met, such as a sufficiently high attendance, referral- and detection rate, a sufficiently high sensitivity and specificity and a relatively low rate of interval cancers and false positive screening results. Screening programs are evaluated based on these parameters, and these parameters have changed over time. The most important changed since the introduction of screening mammography was the increase in recall rate, and thereby an increase in cancer detection rate, screening sensitivity and false positive rate.

Screening outcome can be influenced by a number of parameters including patient (e.g. breast density, previous breast surgery, breast cancer prevalence), imaging (i.e. screen film or digital mammography) and screening situation related parameters (i.e. reading strategy, radiologist performance).

This thesis will explore several factors of the breast cancer screening program in a Southern screening region of the Netherlands with the aim to improve the effectiveness of screening. The main focus of this thesis will be the evaluation of patients with a repeated false positive recall at the transition to digital screening, radiologist's performance at non-blinded double reading and to determine the best reading strategy at double reading.

\section{Re-attendance at biennial screening mammography following a repeated false positive recall}

The first objective of this thesis was to determine the re-attendance rate at screening mammography after a single or a repeated false positive recall (chapter 2). We found that the re-attendance rate for women who had experienced a single false positive recall is significantly worse compared to patients with a negative screen at prior screening mammography (65.4\% versus $93.2 \%$ ). Re-attendance after a repeated false positive recall is even worse $(52.1 \%)$, especially if both recalls comprised the same mammographic lesion (44.3\%).

The second purpose of chapter $\mathbf{2}$ was to determine the effect of the transition from screen film tot digital mammography on the proportion and screening outcome of women recalled twice for the same lesion. Moreover, we assessed the possible influence of comparison with scanned in priors instead of hard copy priors at digital screening on the proportion of women recalled twice for the same mammographic abnormality. We found that, during the first screening round at digital screening, a significantly larger proportion of recalls included women who had been recalled twice for the same lesion and breast cancer was significantly less often diagnosed in these 
women than at screen film mammography, with a concomitant lower positive predictive value (PPV) of recall. In this population, subsequent digital screens were compared with previous digitized analogue screens. Blinded review showed that the availability of the screen film hard copies would have reduced the number of repeatedly recalled women at digital mammography by almost $40 \%$. None of these women were ultimately diagnosed with breast cancer.

We therefore suggest that, in case a woman attends digital screening for the first time after previous screen film mammography (e.g., screening programs currently converting to digital mammography and women who have skipped the digital screening rounds following their latest SFM screen), the screening radiologist should have the opportunity to compare with the previous hard copy screen film examination if repeated recall is considered.

\section{Variations in screening outcome among pairs of screening radiologists at non-blinded double reading}

Substantial inter-observer variability in screening mammography interpretation among screening radiologists has well been documented. However, screening results of pairs of screening radiologists have not yet been published. In chapter 3 we determined the variations in screening performance among unique pairs of screening radiologists at non-blinded double reading. We expected that the addition of a second reader would decrease these variations in screening outcome, especially among a group of screening radiologists who attend quality assurance sessions at the same regular intervals. Nevertheless, our results proved otherwise. Variations in screening performance significantly varied among 26 pairs of screening radiologists with respect to recall rate, program and mammographic sensitivity and PPV of recall. This stresses the importance of monitoring screening results on a local scale.

\section{Blinded versus non-blinded double reading}

European guidelines consider double reading by screening radiologists as standard of reference for the assessment of screening mammograms as this reading strategy significantly increases the cancer detection rate when compared to single reading. Double reading can be performed in a blinded $\left(2^{\text {nd }}\right.$ reader is not informed about the $1^{\text {st }}$ reader's decision) or non-blinded fashion $\left(2^{\text {nd }}\right.$ reader is informed about the $1^{\text {st }}$ reader's decision). In chapter 4.1 we determined the screening mammography outcome at blinded and non-blinded double reading in a prospective population based study. In this study women with discrepant readings between the two radiologists were always recalled for further analysis.

We found that blinded double reading almost doubled the proportion of discrepant readings among recalled women in our study, which resulted in a significantly higher recall rate and false positive rate. However, we found that blinded double reading yielded a significantly better program sensitivity than non-blinded double reading, as 
well as a $14 \%$ higher (7.4 versus 6.5 ), although non-significant, cancer detection rate. We therefore advocate the use of blinded double reading instead of non-blinded double reading.

\section{Diagnostic work-up after blinded and non-blinded double reading}

In the previous chapter we advised the use of blinded double reading over non-blinded double reading. However, the disadvantage of blinded double reading is that it resulted in a higher false positive rate. It is known that the degree of distress after a false positive recall is related to the invasiveness of the assessment. Therefore we compared the diagnostic work-up after blinded and non-blinded double reading in chapter 4.2.

We found that blinded double reading resulted in significantly higher overall (17.4 versus 14.3$)$ and benign biopsy rate (10.1 versus 7.7$)$ than non-blinded double reading. This difference was caused by higher core needle biopsy (CNB) and stereotactic core needle biopsy (SCNB) rates. After non-blinded double reading, a larger proportion of women at benign work-up were evaluated merely by additional imaging. This negative side effect of blinded double reading should be taken into account when adopting this reading strategy.

\section{Arbitration of all discrepant readings at blinded and non-blinded double reading}

Blinded double readings results in discrepant readings. In the previous chapters all discrepant readings were recalled for further evaluation. The objective of chapter 4.3 was to determine the value of adding a third reader for arbitration of discrepant screening mammography assessments.

Discrepant screening mammography occurred more often at blinded (57.2\%) than at non-blinded double reading (29.1\%). Arbitration of all discrepant readings by a third reader would have improved the recall rate and positive predictive value (PPV) of recall at both reading strategies. However, it would have significantly decreased the program sensitivity at blinded double reading. There was no significant effect on the cancer detection rate at both reading strategies and the program sensitivity at non-blinded double reading. This study therefore showed that arbitration of all discrepant screening mammography assessments is a good tool to improve recall rate and PPV, but is not desirable as it reduces the program sensitivity at blinded double reading.

\section{Characteristics of BI-RADS category 0 readings and the effect of arbitration of discrepant BI-RADS 0 readings on screening outcome}

BI-RADS category 0 recalls represent lesions with a low suspicion for malignancy, requiring additional work-up. In chapter 4.4 we evaluated the characteristics of $\mathrm{BI}-$ RADS 0 lesions at blinded and non-blinded double reading and determined the 
potential effect of arbitration of discrepant BI-RADS 0 recalls by a third reader on screening outcome.

We found that both at blinded and non-blinded double reading, 32.0\% and $32.5 \%$ of recalls were assigned BI-RADS 0 with a positive predictive value of $7.2 \%$ and $6.8 \%$, respectively. Compared to non-blinded double reading, BI-RADS 0 recalls at blinded double reading showed a higher discrepancy rate and false positive recall rate. Arbitration of discrepant BI-RADS 0 recalls would have significantly lowered the recall rate, without a decrease in cancer detection rate and program sensitivity. Arbitration would have significantly increased the PPV at blinded double reading. On that account, we advise arbitration of discrepant BI-RADS 0 recalls, at both blinded and non-blinded double reading.

\section{General discussion}

To maintain the effectiveness of breast cancer screening in the rapidly changing therapeutic landscape, it is relevant to continuously evaluate the screening program and try to maximize its benefits and minimize the drawbacks of mass screening. Based on the studies presented in this thesis, the following strategies may improve the effectiveness of the population based breast cancer screening program:

1. If a woman attends digital screening for the first time after previous screen film screening (i.e. screening programs currently converting to digital mammography and women who have skipped the digital screening rounds following their latest SFM screen) the screening radiologist should have the opportunity to compare with the previous hard copy SFM examination if repeated recall is considered.

2. Create specific pairs of radiologists in which radiologists with good to superior screening results are combined with radiologists with less optimal screening results or in which radiologists specialized in breast imaging are combined with radiologists who are not.

3. To detect suboptimal individual screening results or suboptimal results of pairs of screening radiologists, it is important to monitor screening results at a local and individual level.

4. Although a cost effectiveness analysis is still required, blinded double reading with arbitration of discrepant BI-RADS 0 recalls seems the optimal reading strategy to improve the effectiveness of the breast cancer screening program in the Netherlands.

Additionally, a personalized risk-based approach, especially for patients with dense breast tissue, may further improve the efficacy of breast cancer screening in the near future. 


\section{Chapter 6.2}

Valorization addendum 
Breast cancer is the most frequently diagnosed cancer in women worldwide. ${ }^{1}$ Also in the Netherlands, breast cancer is an important threat for public health as the incidence is among the highest in Europe, and still rising. ${ }^{2}$ As in many other countries, the Netherlands has introduced a population based screening program, with the aim to find lesions for which treatment is more effective. The potential harm of breast cancer screening is the creation of unnecessary anxiety and morbidity and inappropriate economic cost. ${ }^{3}$ The Dutch screening program is funded by the national budget and therefore paid with tax revenues. In 2012 the total costs of the program were 64.6 million euro's, which equates to 64 euro's per screening examination. This does not include the costs of positive screening examination, which is paid by the health insurance of the recalled woman.

In this valorization paragraph we make the knowledge obtained from this thesis, available and suitable for economic and social exploitation. The findings of this thesis resulted in novel strategies to improve the efficacy of breast cancer screening program and to minimize the harms, but did not result into commercial products or new business.

In chapter 2 we showed that, during the transition to digital screening, the availability of prior hard copy screen films for comparison would reduce the amount of repeated false positives for the same mammographic abnormality with $40 \%$. Further, we found that patients with a repeated false positive recall for the same mammographic lesion had a significantly worse screening re-attendance (44.3\%) than patients with a negative screen examination (93.2\%) or a single false positive recall (65.4\%).

It is important to keep the number of false positives as low as possible to avoid anxiety and psychological distress among the effected subgroup ${ }^{4-7}$ and to avoid unnecessary work-up costs of benign lesions. Further, the decreased re-attendance rate after a repeated false positive recall may be (partly) due to the tendency to keep women under outpatient supervision after a repeated false positive recall. Prolonged clinical surveillance of women with a previous false positive screen results in additional diagnostic and follow-up costs, compared to mammography performed within the national screening program.

To decrease the amount of distress among screened women and to avoid unnecessary work-up and surveillance costs (financed by the health insurances), we suggest that, in case a women attends digital screening for the first time after previous screen-film mammography screening, the screening radiologist should have the opportunity to compare with the previous hard copy screen-films if repeated recall is considered. This information is relevant for screening programs in other countries currently converting to digital mammography and women who have skipped the digital screening rounds following their latest SFM screen. 
In chapter 3 we found that interobserver variation persisted among 26 pairs of screening radiologists at non-blinded double reading and we suggested to create specific pairs of screening radiologists to optimize screening results. Further we analyzed the breast cancers missed at screening. The analysis of individual and regional screening results, missed cancers and false positive recalls at screening mammography is an important issue of this quality assurance. A radiologist who interprets screening examinations without the opportunity to get feedback on the outcome of recalled women may be hampered to improve his/her recall behavior. This stresses the importance to monitor screening results on a local and individual scale and to provide additional training for screening radiologists with suboptimal screening results or to create specific pairs of screening radiologists. This information is relevant for screening radiologists, screening units and the Dutch Reference Centre for Cancer screening, whom is responsible for ensuring and improving the quality of the breast cancer screening program. The digitization of the screening program and the recently established linkage with the Dutch Cancer Registry database will provide more opportunities to monitor screening results on an individual basis. This further improvement of the quality control of the breast cancer screening program in the Netherlands will eventually improve the breast cancer survival and minimize the breast cancer screening harms.

In chapter 4 we determined the screening outcomes of both blinded and nonblinded double reading and we explored how to cope best with discrepant readings i.e., recall of all discrepant readings; arbitration of all discrepant readings by a third reader; arbitration of only discrepant BI-RADS 0 recalls by a third reader'. We found that blinded double reading with arbitration of discrepant BI-RADS category 0 recalls provides the optimal balance between a high screening sensitivity, relative low recall rate and relative high positive predictive value (PPV) of recall when compared to other double reading strategies.

What does this mean for the women participating in the Dutch screening program? In 2012 there were about 1 million screened women. If the screening mammograms of these women were read in a blinded fashion with arbitration of discrepant BI-RADS category 0 recalls instead of non-blinded double reading with recall of all discrepant readings (the former used reading strategy in our region), this would result in: 700 more screen-detected cancers, 400 less interval cancers and 868 less false positive screening results. This will improve breast cancer survival, as interval cancers have a worse survival, despite receiving more adjuvant chemotherapy, than screen detected cancers. ${ }^{8,9}$ The decrease in false positive screening results will reduce the amount of recalled women with anxiety and distress and reduce the work-up costs of benign lesions. An additional cost effectiveness analysis will be necessary to determine whether blinded double reading, combined with arbitration of discrepant BI-RADS 0 recalls, should become the nationwide standard in the Netherlands. 
In conclusion, this thesis has a societal as well as an economic impact. It offers strategies to improve the efficacy of the breast cancers screening program by detecting more breast cancers at an early stage and thereby reduce breast cancer morbidity and mortality. Further, it offers strategies to decrease the amount of false positive screening results which will reduce the amount of women with anxiety and psychological distress, reduce unnecessary work-up costs of benign lesions, attribute to the maintenance of screening re-attendance and thereby avoid prolonged surveillance costs. 


\section{References}

1. Jemal A, Bray F, Center MM, Ferlay J, Ward E, Forman D. Global cancer statistics. CA Cancer J Clin 2011;61:69-90.

2. Ferlay J, Steliarova-Foucher E, Lortet-Tieulent J, Rosso S, Coebergh JW, Comber H, et al. Cancer incidence and mortality patterns in Europe: estimates for 40 countries in 2012. Eur J Cancer 2013;49:1374-403.

3. Perry N, Broeders M, de Wolf C, Tornberg S, Holland R, von Karsa L. European guidelines for quality assurance in breast cancer screening and diagnosis. Fourth edition--summary document. Ann Oncol 2008;19:614-22.

4. van der Steeg AF, Keyzer-Dekker CM, De Vries J, Roukema JA. Effect of abnormal screening mammogram on quality of life. Br J Surg 2011;98:537-42.

5. Brewer NT, Salz T, Lillie SE. Systematic review: the long-term effects of false-positive mammograms. Ann Intern Med 2007;146:502-10.

6. Bond M, Pavey T, Welch K, Cooper C, Garside R, Dean S, et al. Psychological consequences of falsepositive screening mammograms in the UK. Evid Based Med 2013;18:54-61.

7. Brodersen J, Siersma VD. Long-term psychosocial consequences of false-positive screening mammography. Ann Fam Med 2013;11:106-15.

8. Fong Y, Evans J, Brook D, Kenkre J, Jarvis P, Gower Thomas K. The incidence and 10-year survival of interval breast cancers in Wales. Clin Radiol 2014;69:e168-72.

9. Rayson D, Payne JI, Abdolell M, Barnes PJ, Maclntosh RF, Foley T, et al. Comparison of clinical-pathologic characteristics and outcomes of true interval and screen-detected invasive breast cancer among participants of a Canadian breast screening program: a nested case-control study. Clin Breast Cancer 2011;11:27-32. 


\section{Chapter 6.3}

Curriculum vitae 
Elisabeth Geneviève (given name Lisa) Klompenhouwer was born on February 22, 1985 in Rotterdam, the Netherlands. After graduating from secondary school at the Dendron College in Horst, she studied Medicine at the Maastricht University. She finished her medical study with a scientific internship at the department of radiology of the Maastricht University Medical Center (MUMC), which resulted in her first publication. Immediately after obtaining her medical doctor degree, she started working as a resident in surgery at the Amphia Hospital Breda to gain some clinical experience. On October first, 2010, she started working as radiology resident at the Catharina Hospital Eindhoven and the MUMC (under supervision of dr A.V. Tielbeek and prof dr. M.W. de Haan). She will finish her residency at September 31th, 2015. During her working career she attributed to multiple research projects on breast cancer research, abdominal imaging and interventional radiology. In 2013 she started her research project on breast cancer screening under the main supervision of dr. L.E.M. Duijm, Canisius Wilhelmina Hospital, which resulted in this PhD thesis (promotor prof dr. V.C.G. TjanHeijnen, MUMC). 


\section{Chapter 6.4}

List of publications 


\section{This thesis}

1. Klompenhouwer EG, Weber RJ, Voogd AC, den Heeten GJ, Strobbe L, Broeders MJ, Tjan-Heijnen VC, Duijm LE. Arbitration of discrepant BI-RADS 0 recalls by a third reader at screening mammography lowers recall rate but not the cancer detection rate and sensitivity at blinded and non-blinded double reading. Breast: 2015 June 26 (epub ahead of print)

2. Weber RJ, Klompenhouwer EG, Voogd AC, Strobbe L, Broeders MJ, Duijm LE. Comparison of the diagnostic workup of women referred at non-blinded or blinded double reading in a population-based screening mammography programme in the South of the Netherlands. Br J Cancer: accepted for publication July 2015

3. Klompenhouwer EG, Voogd AC, den Heeten GJ, Strobbe L, Tjan-Heijnen VC, Broeders MJ, Duijm LE. Discrepant screening mammography assessments at blinded and non-blinded double reading: impact of arbitration by a third reader on screening outcome. Eur Radiol. 2015 April 18; (epub ahead of print)

4. Klompenhouwer EG, Voogd AC, den Heeten GJ, Strobbe LJ, de Haan AF, Wauters CA, Broeders MJ, Duijm LE. Blinded double reading yields a higher program sensitivity than non-blinded double reading at digital screening mammography: a prospective population-based study in the south of the Netherlands. Eur J Cancer. 2015 Feb;51(3):391-9

5. Klompenhouwer EG, Duijm LE, Voogd AC, den Heeten GJ, Strobbe L, Louwman MW, Coebergh JW, Venderink D, Broeders MJ. Re-attendance at biennial screening mammography following a repeated false positive recall. Breast Cancer Res Treat. 2014 Jun;145(2):429-37.

6. Klompenhouwer EG, Duijm LE, Voogd AC, den Heeten GJ, Nederend J, Jansen FH, Broeders MJ. Variations in screening outcome among pairs of screening radiologists at non-blinded double reading of screening mammograms: a population-based study. Eur Radiol. 2014 May;24(5):1097-104.

\section{Other}

7. Beek MA, Verheuvel NC, Luiten EJ, Klompenhouwer EG, Rutten HJ, Roumen RM, Gobardhan PD, Voogd AC. Two decades of managing the axilla in breast cancer: A paradigm shift in the south of the Netherlands. Br J Surg: revision pending

8. Beek MA, Gobardhan PD, Klompenhouwer EG, Rutten, HJ, Voogd AC, Luiten EJ. Axillary reverse mapping (ARM) in clinically node positive breast cancer patients. Eur J Surg Oncol. 2015 Jan;41(1):59-63 
9. Kloeze C, Klompenhouwer EG, Brands PJ, van Sambeek MR, Cuypers PW, Teijink JA. Editor's choice--Use of disposable radiation-absorbing surgical drapes results in significant dose reduction during EVAR procedures. Eur J Vasc Endovasc Surg. 2014 Mar;47(3):268-72.

10. Thomassen I, Klompenhouwer EG, Willigendael EM, Teijink JA. Treatment of temporal artery pseudoaneurysms. Vascular 2014, Vol. 22(4) 274-279

11. Klompenhouwer EG, Gobardhan PD, Beek MA, Voogd AC, Luiten EJ. The clinical relevance of axillary reverse mapping (ARM): study protocol for a randomized controlled trial. Trials. 2013 Apr 25;14(1):111.

12. Gobardhan PD, Klompenhouwer EG, Beel MA, Voogd AC, Luiten EJ.Axillary Reverse Mapping. Preserving nodes during an axillary lymph node dissection. Ned Tijdschr Geneeskd. 2013;157(22):A5646.

13. Klompenhouwer EG, Thomassen I, Teijink JA. A man with a bump on his forehead after a fall. Ned Tijdschr Geneeskd. 2013;157(8):A4231.

14. Klompenhouwer EG, Helleman JN, Geenen GP, Ho GH, Vos LD, Van Der Laan L. Reinterventions following endovascular abdominal aortic aneurysm repair: the learning curve of time. J Cardiovasc Surg. 2013 Jun;54(3):367-72.

15. Wegdam-Blans MC, ter Woorst JF, Klompenhouwer EG, Teijink JA. David procedure during a reoperation for ongoing chronic $Q$ fever infection of an ascending aortic prosthesis. Eur J Cardiothorac Surg. 2012 Jul;42(1):19-20.

16. Gobardhan PD, Wijsman JH, van Dalen T, Klompenhouwer EG, van der Schelling GP, Los J, Voogd AC, Luiten EJ. ARM: axillary reverse mapping - the need for selection of patients. Eur J Surg Oncol. 2012 Aug;38(8):657-61.

17. Vainas T, Klompenhouwer EG, Duijm L, Tielbeek X, Teijink J. Endovascular treatment of common hepatic artery pseudoaneurysm associated with upper gastrointestinal tract bleeding. J Vasc Surg. 2012 Apr;55(4):1145-9.

18. Klompenhouwer EG, Gobardhan PD, Verhees R, Peters W, Zoete de JGM. Intestinal ischaemia caused by acute mesenteric vein thrombosis. Ned Tijdschr Geneeskd. 2011;155(44):A3598

19. Klompenhouwer EG, Dings JTA, van Oostenbrugge RJ, Wilmink JT, Oei S, van Zwam $\mathrm{WH}$. Single centre experience of surgical and endovascular treatment of ruptured intracranial aneurysms. Am J Neuroradiol. 2011 Mar; 32(3):570-5.

20. Klompenhouwer EG, Rijken A, vd Schelling G. Trocardhernia's: een zeldzame maar potentieel levensbedreigende complicatie van laparoscopische chirurgie. Ned Tijdschr heelkunde. 2010 Oct;19(7): 262-65 


\section{Chapter 7}

Dankwoord 
Bijna 2,5 jaar geleden gestart aan dit project, waar ik met heel veel plezier aan heb gewerkt! Het combineren van mijn opleiding, promoveren en het heen en weer reizen was een uitdaging, welke ik zonder de hulp van de volgende mensen zeker niet aan had aangekund.

Prof. Vivianne Tjan-Heijnen, mijn promotor, jouw fris blik en snelle reacties zijn heerlijk om mee samen te werken! Merci voor alle goede begeleiding en waardevolle inbreng.

Mijn co-promotoren, Lucien Duijm, Mireille Broeders en Adri Voogd, alle drie van onmisbare waarde voor mijn proefschrift.

Lucien, de machinist van de borstkanker screening trein, zonder jou was dit proefschrift er zeker nooit geweest. Waar ter wereld je ook was, reactie op mijn mails kreeg ik altijd a la minute. Ontzettend bedankt dat ik de mogelijkheid heb gekregen om bij je te promoveren. Jouw enthousiasme en deskundige commentaar van waar ook ter wereld, maken je de beste begeleider die een promovendus zich kan wensen.

Mireille, bedankt voor je oneindige deskundigheid op gebied van borstkanker screening. Jouw ideeën en stimulerende commentaar hebben mijn proefschrift zeker beter gemaakt.

Adri, we kennen elkaar al sinds het opzetten van de ARM-studie. Ik was dan ook erg verheugd toen ik begreep dat jij ook onderdeel van mijn promotie team zou zijn. Bedankt voor al je betrokkenheid, correcties, kennis en adviezen. Ik hoop dat onze samenwerking nog lang mag voortduren.

Prof. Ard den Heeten, bedankt dat ik als mede auteur gebruik mocht maken van al je kennis en inzichten op het gebied van de borstkanker screening.

De leden van de beoordelingscommissie, Prof. Dejong, Dr. Lobbes, Dr Pijnappel en Prof. Verbeek, ik wil jullie hartelijk bedanken dat jullie de tijd hebben genomen voor de inhoudelijke beoordeling van mijn proefschrift.

Mijn paranimfen Renee en Mirjam.

Mirjam, mijn lieve kleine zusje, mijn maatje. Renee, mijn fantastisch lieve en trouwe vriendin. Bij jullie kan ik altijd terecht voor adviezen, een feestje en een traan. Jullie hebben me oneindig gesteund bij het combineren van mijn opleiding, privé leven en onderzoeksambities. Het is bijzonder hoe jullie als advocaat zoveel weten van mijn vakgebied. Merci voor alles meiden. Met jullie naast me wordt deze dag met zekerheid een succes! 
Mijn voorgangers, Vivianne, Wikke en Joost. Ik heb ontzettend veel gehad aan jullie ervaring en inzichten. Joost, bij jou kan ik altijd terecht met al mijn (minder) intelligente vragen of gewoon een goede grap. Ik ga al onze mooie discussies missen. Roy en Rob, succes met jullie verdere promotie traject.

Mijn dank gaat naar de overige mede auteurs van dit proefschrift, Luc Strobbe, Marieke Louwman, Jan Willem Coebergh, Dick Venderink, Frits Jansen, Ton de Haan, Carla Wauters.

Dr. Tielbeek en Harrie, mijn opleiders. Jullie betrokkenheid bij de voortgang van mijn opleiding, promotie, banen keuze maar ook zeker bij mij als persoon maken elke werkdag in het Catharina ziekenhuis hartverwarmend en één groot feest.

Leden van de maatschap radiologie uit het Catharina ziekenhuis, dokter Jurgens, Tielbeek, Tjon, Wondergem, Frits, Astrid, Harrie, Alette, Rianne, Menno, Wikke, Lonneke en Joost, zonder al jullie screeningswerk was dit boekje er nooit geweest. Bedankt dat jullie mee hebben gewerkt aan al mijn verschillende leesmethodes. Daarnaast wil ik jullie ontzettend bedanken voor alle interesse, steun en enthousiasme, dit heeft mijn promotietraject veel makkelijker gemaakt!

Collega arts-assistenten, de afgelopen 5 jaar een wisselende groep, maar altijd even leuk! Ik ga jullie missen.

Lieve Julia, mijn roomie, met de rust en gezelligheid bij ons in Eindhoven, was het afmaken van mijn proefschrift een makkie! Gelukkig nu alle bij richting de Randstad, zodat ik je niet zoveel hoef te missen.

Lieve oma, papa, mama, Chris, Mirjam en Julia, de laatste jaren heb ik jullie minder gezien dan ik zou willen. Jullie zijn ontzettend belangrijk voor me! Bedankt voor alle interesse, begrip en steun.

Lieve vriendinnetjes Janneke, Jordy en Renée, aan jullie hoef ik niet veel worden vuil te maken, want in onze vriendschap spreek alles voor zich. Jullie zijn simpelweg de beste vriendinnen die iemand zich kan wensen!

Mijn aller liefste Rik, dat ik jou heb mogen ontmoeten is het grootste geschenk in mijn leven. Jij bent de handrem dit zorgt dat deze workaholic zich niet te pletter rijdt;). Met jou aan mijn zijde is elke dag een feestje. Ik kan niet wachten om al onze toekomst dromen te laten uitkomen! 